\title{
Constructing the Deaf Sense-of-Self: The Tug-of-war Between Language and Technology in the Construction of Deaf Identity
}

$$
\text { by }
$$

\section{Celia J. Posner}

A thesis submitted to the Faculty of Graduate and Postdoctoral Affairs in partial fulfillment of the requirements for the degree of

\section{Master of Arts}

in

Applied Linguistics and Discourse Studies

\author{
School of Linguistics and Applied Languages \\ Carleton University \\ Ottawa, Ontario
}

\author{
(C) 2012
}

Celia Posner 
Library and Archives

Canada

Published Heritage

Branch

395 Wellington Street

Ottawa ON K1A ON4

Canada
Bibliothèque et

Archives Canada

Direction du

Patrimoine de l'édition

395 , rue Wellington

Ottawa ON K1A ON4

Canada
Your file Votre référence

ISBN: 978-0-494-87794-4

Our file Notre référence

ISBN: 978-0-494-87794-4

\section{NOTICE:}

The author has granted a nonexclusive license allowing Library and Archives Canada to reproduce, publish, archive, preserve, conserve, communicate to the public by telecommunication or on the Internet, loan, distrbute and sell theses worldwide, for commercial or noncommercial purposes, in microform, paper, electronic and/or any other formats.

The author retains copyright ownership and moral rights in this thesis. Neither the thesis nor substantial extracts from it may be printed or otherwise reproduced without the author's permission.
AVIS:

L'auteur a accordé une licence non exclusive permettant à la Bibliothèque et Archives Canada de reproduire, publier, archiver, sauvegarder, conserver, transmettre au public par télécommunication ou par l'Internet, prêter, distribuer et vendre des thèses partout dans le monde, à des fins commerciales ou autres, sur support microforme, papier, électronique et/ou autres formats.

L'auteur conserve la propriété du droit d'auteur et des droits moraux qui protege cette thèse. $\mathrm{Ni}$ la thèse ni des extraits substantiels de celle-ci ne doivent être imprimés ou autrement reproduits sans son autorisation.
In compliance with the Canadian Privacy Act some supporting forms may have been removed from this thesis.

While these forms may be included in the document page count, their removal does not represent any loss of content from the thesis.
Conformément à la loi canadienne sur la protection de la vie privée, quelques formulaires secondaires ont été enlevés de cette thèse.

Bien que ces formulaires aient inclus dans la pagination, il n'y aura aucun contenu manquant. 


\begin{abstract}
This thesis examines the formation of social identity among six deaf or hard-ofhearing people, aged between 20 and 35 years old. This qualitative research uses data collected during interviews to examine the process of identity formation, focusing on family, friends, school, language use and technology as factors involved in its construction. The study also examines the intricacies of the relationship between identity and group membership. The findings indicate that each of these components play a part in the participants' identities, but to differing degrees. It is also apparent from the results that identity and group membership have a very complicated relationship that depends on the above factors, but that it is very difficult to predict how identity will affect feelings of group membership among the participants. In addition to these conclusions, it becomes apparent that technology plays an important part in a deaf/HoH person's identity construction because it facilitates the cultivation of multiple and contrary identities.
\end{abstract}




\section{Acknowledgements}

I would like to thank, first and foremost, my supervisor Dr. Devon Woods. The value of your help and guidance through this is immeasurable. Thank you. I would also like to thank the members of my defence committee, Dr. Jaffer Sheyholislami and Dr. Timothy Pychyl for their comments and feedback.

To Dr. Lynne Young: thank you for your help in preparing for my defence and also for being an additional audience member.

For their support on campus, for making my life as a student and a faculty member infinitely easier, I would like to thank Joan Grant and Tracey Wright. You are truly indispensible.

To my participants: thank you for providing me with ample new knowledge, for letting me into your lives and for opening my eyes to previously unknown territories. Your stories were inspirational and riveting.

To Jon Kidd: thank you for providing me with a base and for sharing your fascinating stories. The conversations we have had over the years were the inspiration behind this project.

To my parents and brothers: thank you for your never-ending support, and for lending an ear more than once or twice. There were times when our talks were all that kept me motivated.

To my dear friends Ari Black and Andrea Balon, you were both absolutely necessary in this process. Ari, your feedback and support always came at the right time, and even when it was hard to hear, I appreciated every word. The balance you also provided - with off-topic talks - was also invaluable to me. Andrea, you are a brilliant force in my life and your support and feedback were also invaluable to me. Working with you in the last leg of writing was simply exactly what I needed. Thank you both. 


\section{Table of Contents}

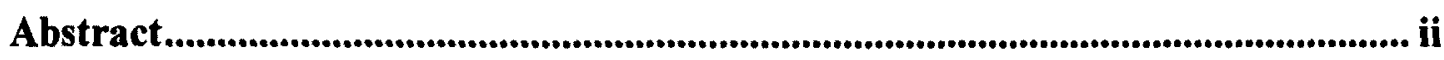

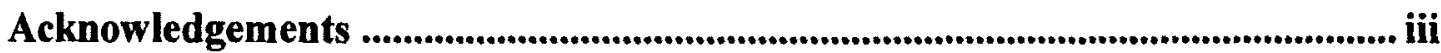

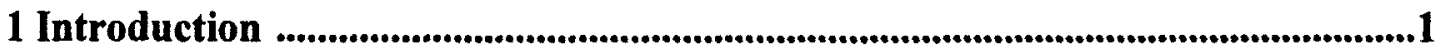

2 Literature Review ....................................................................................................9

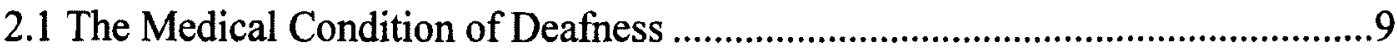

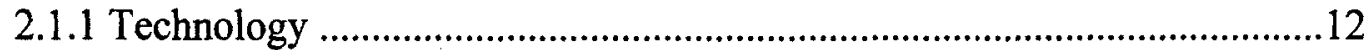

2.1.2 Cochlear Implants: Then and Now ...................................................13

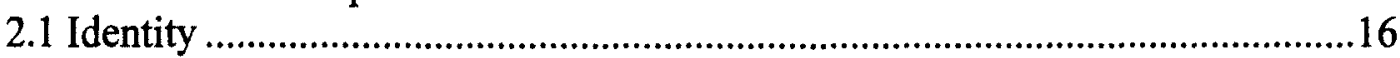

2.2.1 What is Identity? ........................................................................16

2.2.2 How is Identity Constructed?...........................................................18

2.2.3 Identity and Early Socialization...........................................................18

2.2.4 Friendship and Group Membership .......................................................20

2.2.5 Language Ideologies, Minority Languages, and Identity ........................22

2.2.6 Multiple and Contradictory Identities ...................................................24

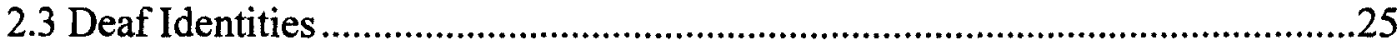

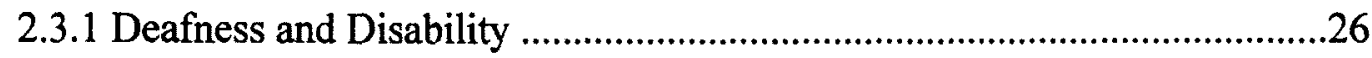

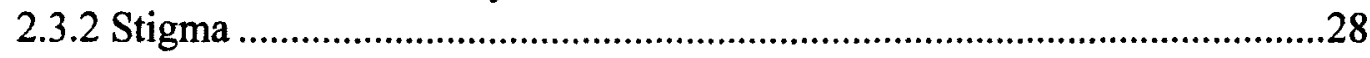

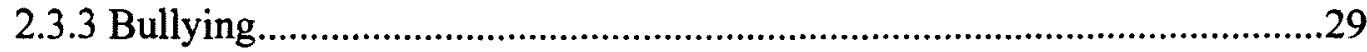

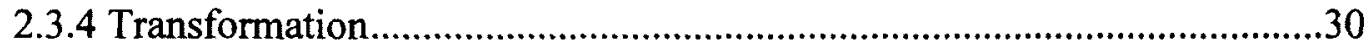

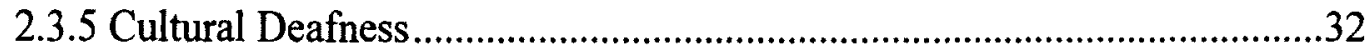

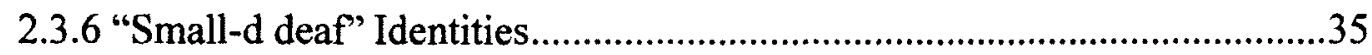

2.3.7 Deaf Identities, Sign Language and Language Ideologies.........................38

2.3.8 Deafness, Language and Education ......................................................41

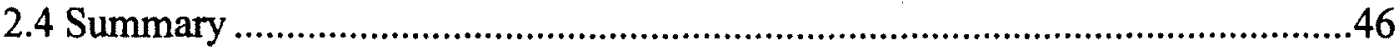

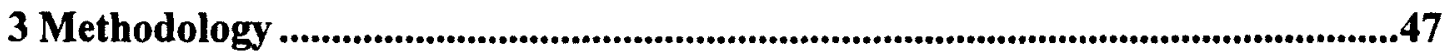

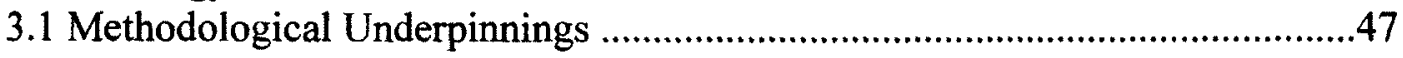

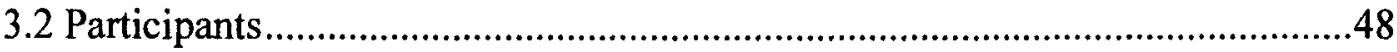

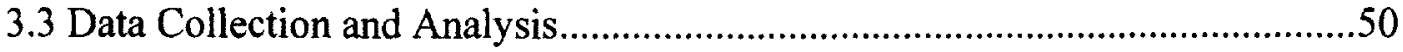

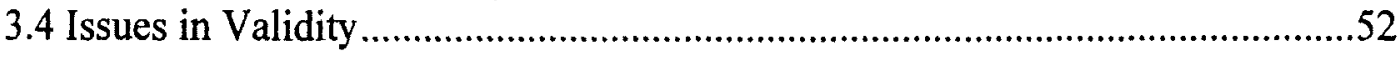

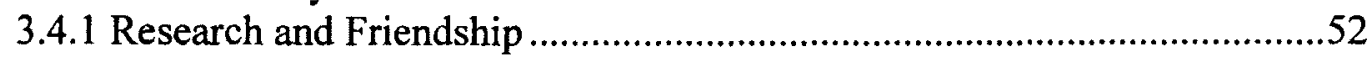

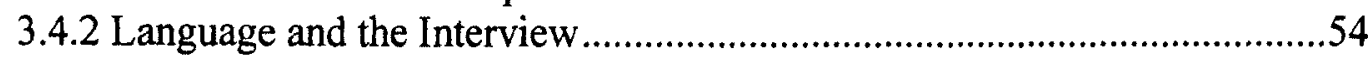

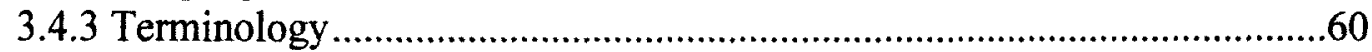

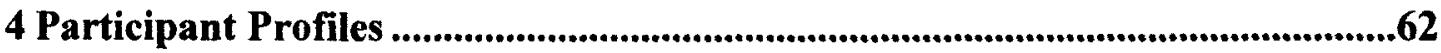

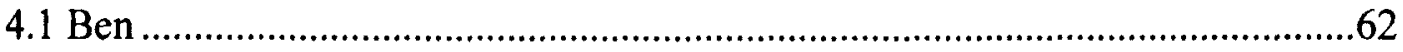

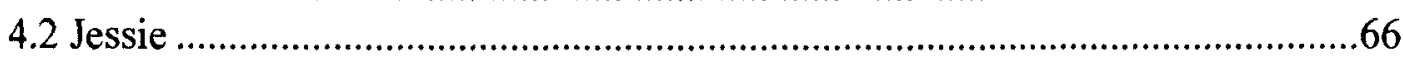

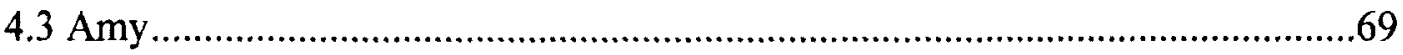

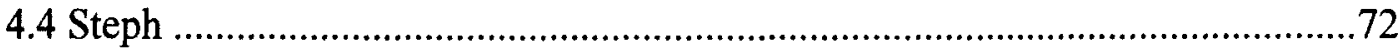

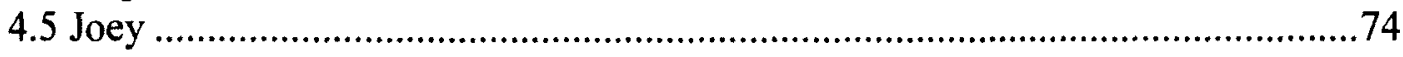

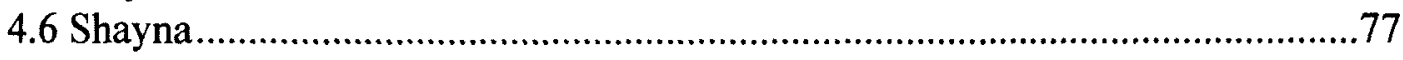

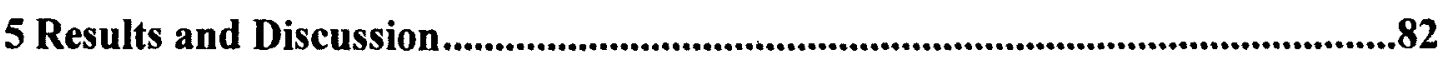

5.1 Early Relationships and Communication...................................................85 


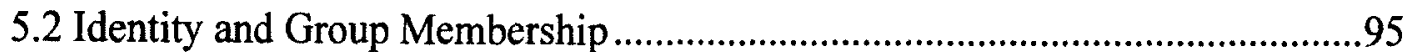

5.3 Changes in Auditory Context................................................................... 102

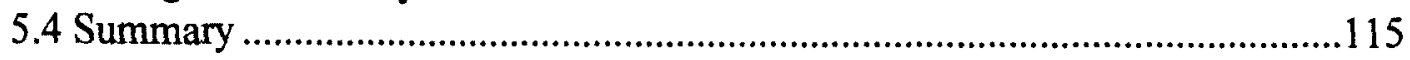

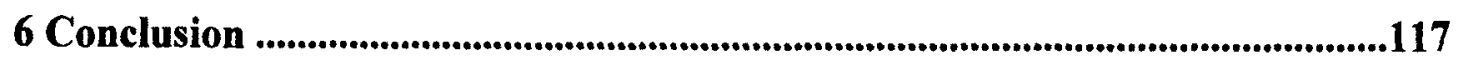

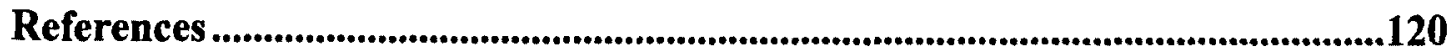




\section{Introduction}

Since 2005, when I first started learning American Sign Language (ASL), I have been involved in Deaf culture and in the Deaf community. By a series of unusual situations, which included circumstances that demanded a much higher level of proficiency than I had attained, I became fascinated by the language and by its users. Visual languages afford their users different social and pragmatic norms than spoken languages, arguably due to their modality difference, and these distinctive conventions are what cemented my passion for the language.

Over the years, as I have met people who are deaf or hard-of-hearing $(\mathrm{HoH}), \mathrm{I}$ have often been struck by their confidence and the ease with which they maneuver in this world. Their attitudes towards their deafness has convinced me that deafness is not the disability we hearing people, as the larger, dominant group in society, make it out to be ${ }^{1}$. This observation may seem odd, but I point it out to set it against the prevalent assumption that surrounds deafness. For most of the hearing world, a hearing impairment, as the term underlines, is seen as a deficiency that presumably makes life very difficult for the afflicted. Accommodations (meant to acculturate the deaf person to the dominant majority) provided in areas such as education and accessibility, are often hit-or-miss, resulting in a wide variety of outcomes. Some individuals affected by hearing loss manage to assimilate into the dominant culture relatively easily, while others all but shun it, opting instead to live as much as possible in the Deaf world.

\footnotetext{
${ }^{1}$ As I do not subscribe to the assumption that deafness means disability, I will avoid using terms such as hearing impairment as often as possible, however, there are times when this term is necessary, particularly when referring to the stereotypes surrounding deafness. This issue is further discussed in chapter 3.
} 
Some also believe that ASL is the deaf person's natural language, but this notion is not common among the general public. Due to the language's stigma, it tends to be associated with a relatively low level of prestige. As such, ASL cannot be classified as a simple minority language because it is associated with a group that is seen as a disabled minority. The language is stigmatized to the extent that to be a learner is to be seen as compassionate and benevolent, and a deaf user is assumed to be unable to use "normal" language; hearing users are often assumed to be working towards a career goal that involves helping the deaf community or its members in some way. Learners of other foreign languages are not assumed to have such motivations; no spoken language, regardless of its social standing, is discredited in quite the same way. As a teacher, I have encountered a substantial number of students motivated to learn ASL by the promise of higher wages or better employment opportunities, rather than by a genuine interest in the language and the people who use it.

What these students and much of the larger population do not realize is that, members of the Deaf community see themselves as part of a cultural group. The heart of their culture hinges on shared values and beliefs. Members of this group support one another in their attempts at finding a solid footing in the greater community, while retaining a Deaf identity. A Deaf identity is one held by a person whose hearing is diminished, but is content with their hearing status.

Carty (1994) explains that hearing loss is a crucial factor in the identity development of a Deaf person, and that Deaf identity has certain defining characteristics, such as the acceptance of deafness as a central and positive part of one's being. Baker (1999) further stipulates that this sense of identity is not simply defined by the lack or 
loss of hearing itself, but rather in the attitude towards one's own deafness. In other words, it does not necessarily follow that severe hearing loss correlates with a stronger feeling of belonging in the Deaf community; individuals with relatively little hearing loss may self-identify as Deaf, and vice versa. Based on this suggestion, it would seem that lack or loss of hearing plays a part in identity construction for the hearing impaired but also that this is not the only factor involved in the process. What other factors are of importance, then?

Family background might play an important part in the process of identity construction. Most deaf children are born into hearing families and in these instances it is often the case that Sign Language is not used at home. As deaf children struggle to acquire spoken language, it is conceivable that these children have limited means of communication at home. This could be a problem as communication is imperative for the creation of bonds between people.

School - by virtue of being an environment in which a major part of many of our lives are spent - should play an important role in determining which groups we feel at one with, and therefore, how we come to view ourselves. Deaf children may be put in the mainstream education, where the language of instruction is the dominant one of the area and deaf children are put in a position where they must use technology of some sort to keep up. Or, they may go to residential schools for the deaf, where Sign Language is the language of instruction and auditory accommodations are unnecessary. I wondered whether the language of this prominent environment played an important role in identity construction, and also whether hearing impaired children would have a clearer sense of 
self if they were educated in a modality that suits their strengths. As well, when deaf children were made to use technology, this too might affect identity formation.

In addition to issues related to the language of instruction and the accommodations provided in schools, deaf children's social circles may be different than those of their hearing peers. Being disadvantaged as they are in the mainstream could lead to troubled peer relations and a lack of friendships. It stands to reason that any difficulties in early social relations - both within the family and among peers - could have an effect on adult relations as well.

Since most deaf children come from hearing families, where they are not exposed to Sign Language (Gallaudet Research Institute, 2006), they often are not exposed to Sign Language at all until they take it upon themselves to learn it. In that situation, it might be too late to have an effect of those deaf peoples' identities; on the other hand, perhaps being exposed to Sign Language, regardless of when, has a profound effect on deaf peoples' identities and therefore, group membership proclivities.

Finally, as mentioned above, the use of technologies might have an influence on the users of such devices. Deaf people have access to a variety of hearing amplification hardware that may allow them to make use of their residual hearing. As such, it is possible that the fact of wearing hearing aids, or other such devices (a fuller discussion follows), has an impact of the formations of their identities. In fact, the cochlear implant, a very trendy and permanent hearing device may have a very strong effect on identity as it fundamentally changes the wearer by virtue of being implanted into the body.

When I first started the undertaking of this project, my idea was to explore the issue of identity among the Deaf population, albeit on a much smaller scale (I had never 
intended to attempt a large-scale quantitative study). I imagined at the time of project conception that I would interview some members of the Deaf community and that they would support my notions of how they came to construct their respective senses of self. In an effort to tie the project to my field of study, I built into it the suggestion that there was a correlation between the language of instruction at the primary and secondary levels, and a sense of identity or belonging with either the hearing or the deaf world. Superficially, it seems that this is the case for some. In the process of selecting participants, I stayed close to home and recruited people with hearing impairments with whom I had easy access. Being a teacher of American Sign Language at a university, I have had the occasional hearing-impaired student enroll in my classes; these were some of the people I sought out to participate.

Much of the literature on Deafness calls Sign Language the "natural" language of the Deaf community. By this, it is meant that those who embrace their deafness are naturally more comfortable with visual language and therefore prefer it to spoken language. In my mind, I had extrapolated this notion to such a degree that I assumed that those hearing impaired persons who had not grown up exposed to a visual language would, once enlightened, choose the Deaf community as their true family. In fact, I was fairly certain that my former students would share with me their feelings of awakening; that they would reveal to me their goals to join this group, with whom they shared important parallel experiences. This did not happen.

There is a relatively substantial body of literature that presents issues relating to Deaf identity, Deaf education, Sign Language and Deaf culture. What I found during this project, however, is that the discourse community is solely focused on those hearing 
impaired people who identify as culturally Deaf. There has not been emphasis on the lifestyle choices and identities of deaf people who wish to remain part of the majority hearing culture, or are attempting to straddle the two cultures. These people are seen as disabled by society at large and although they do not always agree with the label, they are certainly disadvantaged. Nonetheless, they do not seek refuge from the challenges associated with deafness by joining the Deaf community, even though this would offer them a buffer against these difficulties.

I have long known that some deaf individuals choose to be active members of the hearing world and not to identify as Deaf. Conversely, some hearing persons opt to identify at least partially with the Deaf community, once introduced to the language and the group. Intuitively, one might expect that individuals would choose to identify as belonging to a group with which they find similitude and yet, superficially, this does not seem to be the case in every instance.

In fact, as I will present in this thesis, this happens within my group of participants, and hearing technologies tend to be the facilitators of such migrations. With the technological advancements of cochlear implants, some have raised concerns about the "safety" of Deaf culture. Some have warned that the prevalence of such a device may essentially put an end to what remains of Deaf culture. This concern is intricately tied to issues of identity: it assumes that the eradication of hearing impairment will also mean the dissolution of the self-label Deaf.

This concern is not without foundation. In the late summer of 2010, the Provincial Government of Newfoundland and Labrador announced that the Newfoundland School for the Deaf (NSD) would be closing due to the fact that no students would be attending 
in that coming year and there were no new enrolments projected for the following five years (Government of Newfoundland and Labrador, 2010). The problem was not that deaf students ceased to exist suddenly, but rather that all 199 deaf students in the province had been fitted with hearing devices (primarily cochlear implants) and were now being mainstreamed.

There was general uproar from the Deaf community in response to the news release. Many felt that the school's closure was a step backwards in education and that this, sadly, would further chip away at Deaf culture. The Deaf community sees residential schools for the Deaf as cultural hubs, where, very often, pupils are exposed to their culture for the first time. By closing NSD, the Provincial Government was putting a stop to the growth of Deaf culture in Newfoundland. And it follows, then, that Deaf identity being related to culture - would be threatened as well.

In this study, the personal histories of six deaf/ $\mathrm{HoH}$ persons were explored with the intention of learning what goes and went into the formation of their identities. My research questions are as follows: how have these individuals constructed their identities? And what factors and experiences came into play in this process?

In this thesis, I attempt to shed some light on some of the possible answers to these questions. Since this project is fundamentally exploratory in nature, and the research undertaken to delve into these topics is qualitative, I do not venture generalizations; instead, I present the perspectives of my participants in an effort to contribute to the discourses surrounding deafness and identity.

The next section of this paper presents a survey of this discourse by first introducing the technicalities of deafness, of identity and then of deaf identity. Section 
three describes the methods involved in this study, leading to a report of the participants in section four. In the final section, I discuss the findings and their relevance to the topic, and, finally, the main technological innovation that allows those with hearing loss new choices in terms of how they construct their identity, cochlear implants. 


\section{Literature Review}

This chapter offers an explanation of the broad themes underlying this project. Each of the themes presented here are related to one another, in sometimes quite complex ways. The chapter is organized so as to first lay a theoretical foundation for a discussion of deaf identity, which encompasses all the earlier themes laid out in this chapter.

The first section provides an explanation of the technicalities of deafness and of the technologies that were developed in response to the condition. The second section centers on identity: what it is, what experiences and social situations are relevant to its construction, and the idea that humans are made up of more than one identity. Each of the sub-sections begins with an account of the issues in general and subsequently narrows in on how deafness might affect each process. Finally, the last section of this chapter is the culmination of all the themes presented prior. It gives an explanation of deafness as a disability, and what social factors come into play for deaf peoples' constructions of identity. Then, an explanation of group membership options is presented, with an eye to how this affects identity formation (and the possible options available to a deaf person). The discussion then turns to the role of languages on deaf identities and, lastly, on education and deafness.

\subsection{The Medical Condition of Deafness}

Deafness takes varying forms, audiologically speaking. In other words, there are different types of hearing loss. Conductive hearing loss is a result of a malfunction in the outer parts of the ear, where the sound does not travel from the outer ear canal to the middle ear. This type of hearing loss can usually be corrected through medical or surgical procedures. Sensorineural hearing loss is a result of damage to the inner ear (cochlea) or 
to the auditory nerve. This type of hearing loss is often not correctable by medical or surgical means; however, cochlear implants are specifically meant to address this type of condition (American Speech-Language Hearing Association [ASHA], 2011). Hearing loss is measured by performing a test called an audiogram, which gauges the lowest decibel levels a person can detect. The ranges are as follows:

\begin{tabular}{|l|l|}
\hline Degree of hearing loss & Hearing loss range (dB) \\
\hline Normal & -10 to 15 \\
\hline Slight & 16 to 25 \\
\hline Mild & 26 to 40 \\
\hline Moderate & 41 to 55 \\
\hline Moderately severe & 56 to 70 \\
\hline Severe & 71 to 90 \\
\hline Profound & $91+$ \\
\hline
\end{tabular}
(ASHA, 2011)

The above figures are put in perspective by noting that a normal conversation usually occurs between $30-60 \mathrm{~dB}$, where the lower end refers to a whisper and the higher end indicates a conversation taking place in a moderately noisy environment. The noise level a subway makes is approximately $90 \mathrm{~dB}$ and discomfort for someone whose hearing is in the normal range usually starts around $120 \mathrm{~dB}$ (Able Hearing Pty. Ltd., 2010).

The specifics of hearing loss can also vary in a number of ways. A person may have unilateral loss - where hearing is affected in one ear only - or bilateral loss. They may have symmetrical or asymmetrical loss, where the degrees of loss are different in each ear. Hearing loss may occur suddenly or progressively, such as with gradual onset deafness. Adults who lose hearing later in life (late-deafened adults) also experience progressive loss. Finally, loss may also be stable or fluctuating, at times getting worse or even better (ASHA, 2011). 
Causes of deafness are also varied. Congenital deafness is present at birth and may be related to genetic or nongenetic factors. Nongenetic factors include exposure to toxins, viruses or complications at or before birth; genetic factors may be related to a number of syndromes, such as Down syndrome or Usher syndrome (ASHA, 2011). Acquired hearing loss happens after birth and is related to illness. Along with exposure to medications that are toxic to the ear, and other traumas, the auditory nerve can be damaged by a number of illnesses that cause a high fever, such as meningitis or measles (ASHA, 2011). Deafness that occurs before the age of 3 years old is said to be prelingual, meaning children who are diagnosed, or become deaf, in this window will not be equipped with language before the loss occurs (Smith \& Campbell, 1997).

The medical community values the use of technology to help remedy a person's hearing loss. There are different options available to those who seek to augment their hearing capabilities. Hearing assistive technology refers to a collection of devices that typically consist of a microphone and a receiver used together, but which are physically separate. That is, the microphone can be installed in, or worn by, the source of the sound, while the deaf person wears the receiver. FM systems are part of this category and are often used in schools. Hearing aids are worn by the deaf person, on the ear, and amplify the sounds around so that a person may make use of their residual hearing. Some deaf people combine the use of hearing aids and assistive technologies. Finally, cochlear implants are another option. These are surgical and consist of an external and an internal part, which is implanted in the inner ear (ASHA, 2011). For an adult to be implanted they must normally have a degree of hearing loss of severe to profound; a child must normally 
have a degree of hearing loss of profound (ASHA, 2011). A more detailed explanation of the cochlear implant will be presented below.

\subsubsection{Technology}

In the last 30 years, there have been substantial advances in medical findings related to technology and the ear. These have resulted in the creation and dissemination of the cochlear implant, a very permanent transformative tool. These advances have had widespread effects, influencing deaf identity and important ways (this will be explored in the final sections of this chapter).

The effects of the cochlear implant are generally more potent than those of less invasive technologies (hearing aids, for example) because it is targeted at people with a higher degree of hearing loss and because it is implanted into the body and cannot be removed. The rise in the use of cochlear implants in particular has affected the way we conceive of deafness: while it was once seen as a defect that could not be remedied, the advances in technology have turned deafness into a 'curable' disorder, in the eyes of some, particularly the medical community (Shultz, 2000). They have changed the nature of deafness as a medical condition by augmenting the hearing of many people with hearing loss. This push towards accepting deafness as a disability that can be, to some extent, corrected, has reinforced the lay concept of deafness and threatens the concept of Deaf culture, thus having a strong effect on notions of identity for deaf people (both these themes will be treated in section three of this chapter). These advances have also caused the Deaf community to come together to denounce the use of cochlear implants, especially when implanted in young children (Lane \& Bahan, 1998). Finally, it has also changed the nature of education for deaf children. While they were previously 
encouraged to attend Schools for the Deaf, deaf children are now primarily made to use technology and become as 'hearing' as possible (as evidenced in the anecdote included in the previous chapter).

Presently, a brief history of the cochlear implant is offered, since this mechanism has had such a salient effect on deaf identity, which has led to contentious debate in the Deaf community.

\subsubsection{Cochlear Implants: Then and Now}

Over 200 years ago, Alessandro Volta inserted metal rods connected to a battery, into his ears and sent a jolt of electricity into his head. He described what he heard as a "jolt in the head". This experiment indicated that, electrical currents in the ear canal can be perceived as sounds by the brain. Throughout the years that followed, various similar experiments were conducted. Finally, in 1957, two researchers found that an electrode near the auditory nerve allowed a patient to discriminate some sounds and words. This led to true progress in this area (Spencer \& Marschark, 2003).

In the 1970 s, a single electrode cochlear implant was found to provide some help to patients; the device was approved for adult implantation in 1984 (Estabrooks, 1998), but when it became apparent that the benefits to patients were limited, the makers of the device ended manufacturing and pulled the product from the market (Spencer $\&$ Marschark, 2003).

During the late 1970 s, more complex cochlear implant systems were also being developed. These were multichannel devices, which provided the auditory nerve with stimulation in multiple locations, via the cochlea. These had become quite popular by the late 1980s. By this time, multichannel cochlear implants had already begun to be tested 
on babies. By the mid-1980s, both children and adults were being implanted, although the FDA did not formally approve the implantation of babies until 1990, and stipulated that a child must be at least two years old to undergo the surgery (Spencer \& Marschark, 2003).

In 1998, the FDA approved the implantation of babies at least 18 months old and lowered that to 12 months old in 2002 . Today, babies younger than a year are being implanted at the discretion of the surgeon (Spencer \& Marschark, 2003). The motivation for early implantation is related to its demonstrated effectiveness within this age group (Colletti, Carner, \& Colletti, 2006; Economides, Parpounas, Koudoumnakis, Vagis, Choulakis, \& Economides, 2006; Nikolopoulos, O'Donoghue, \& Archbold, 1999).

Cochlear implant technology was initially intended to reinstate some hearing for late-deafened adults, but the expectations quickly shifted and parents started to rely on Cl's to aid in deaf children's speech perception and development. As the objectives of implantation shifted, hostility grew within the Deaf community. Arguments against implants included the limited effectiveness of the devices, the interference they caused with regard to the child's identity as a Deaf person and the issue of using public funds to aid individual deaf children while the general deaf public continued to receive limited access to other technologies (Christiansen \& Spencer, 2002; Spencer \& Marschark, 2003).

One of the greatest preconceived notions about Cl's is that they make deaf people into hearing people. In fact, results of implantations vary widely (Niparko \& Blankenhorn, 2003) and the technology today still cannot reinstate "normal" hearing (Shultz, 2000), nor can it mimic exactly what a hearing person hears, despite significant advances in technology. For this reason, the argument made by the Deaf community 
regarding the development of identity is especially relevant. The cochlear implant does not render the child hearing, yet an implanted child is different than a non-implanted deaf child. If the event of implanting a child plays a part in their exclusion from the Deaf community, with whom they share fundamental physiological similarities, but still does not make them like the majority group, this device is arguably unethical. In actuality, a CI may remove the deaf child from either group and supplants them in a new, even smaller minority.

In examining the effects of CIs on language acquisition in children, Wheeler et. al. (2007) report that their participants showed a significant preference for spoken language, and although the authors point out that their respondents were "flexible in their use of language and mode" (p. 310), none of these participants chose to make use of the British Sign Language interpreters available. In fact, the "languages" these authors refer to are English and sign-supported English - both of which are forms of only one language. This is significant because, although proponents of the $\mathrm{CI}$ attempt to represent it as inclusive (as in this article), children with CIs are not flexible in their language modalities. Claiming that these implanted children showed flexibility in language use, gives the erroneous impression that they were part of various groups, when in fact, they were comfortable with only one language.

The Deaf community fears cochlear implants because they are powerful hearing devices that presumably keep the wearer in the hearing world. In the film, Sound and Fury (2000), an interviewee voices a concern mirrored by the Deaf community in general, in response to the popularization of cochlear implants. He explains that an influx in implantations will result in a sub-group of deaf people likened to robots: these people will 
neither fit into mainstream society, nor will they fit into the Deaf community. They will not only be different from Deaf people, they will also differ from hard-of-hearing people.

Shilling (2005), quoting Featherstone (1995), explains, “"technological modes of interchange' can provide a substitutional corrective to the body's limitations and 'open up new possibilities for intimacy and self-expression"” (p. 188). The Deaf community's concerns, however, contradict what Featherstone asserts. Cl's may open up new possibilities for intimacy, but they can also limit the pool of potential connections by stigmatizing those who wear them, thereby influencing the identities of the deaf people who make use of this technology.

\subsection{Identity}

Identity is discussed in a variety of disciplines, which speaks to its relevance in general. Identity refers to an organization of beliefs about our 'self' and these beliefs are important because they make up our personal realities (Limaye, 2004). It is believed by some that identity is responsible for the way we feel about ourselves or conversely, that an uncertain sense of identity can lead to the loss of our sense of well-being, emotionally or physically (Simon, 2004).

\subsubsection{What Is Identity?}

The concept of identity can be unpacked and separated into a few categories but each of the variances in definitions amount to how we situate ourselves in our world(s). Woodward (2004) distinguishes between personal and social identities. He explains that social identity requires the conscious and active decision to align oneself to a group, or to an identity trait. While personality (sometimes called personal identity, as above) describes internal characteristics, social identity refers to those characteristics we choose 
to associate to our being. However, in order to construct our personalities, or personal identities, there must still exist a process of comparison - something inherently social, as it requires others. Therefore, identities of all forms are fundamentally social because they are made up of external characteristics, which are essentially social in that the meanings attributed to them are negotiated and agreed upon by society, or members of a community or group. In other words, identity is formed with the help of others. As Pittam (1999) explains, "[it] may be established collaboratively among participants in an interaction; it is in part a negotiated concept" (p. 116).

Identity is not only thought to be a result of our interaction with the world but also to dictate how we interact with the world (Simon, 2004). As it stems from our interactions with this world, perceptions are central to identity constructions: the input from our environment is what triggers the process of identity-formation. Events or situations are interpreted as input, which we process either consciously or automatically (Burke \& Stets, 2009), and the results of our processing amounts to a piece of the puzzle. Each piece contains information about judgments we have made in response to input.

These judgments, however, are dictated at least in part by cultural and societal norms. These norms dictate how symbols are assigned meanings, which are mutually comprehensible to groups of people. While other cultures may distinguish between the "self" and the "not-self" differently than in Western society, the latter tends to associate a healthy sense of self with a clear-cut division between the internal and the external. "From a sociohistorical point of view, the self is a cultural theory or set of beliefs about the person. [...] As we mature, we learn our culture's theories about the world, other people, and even ourselves. Different cultures have different theories" (Sampson, 1991, 
p. 210). Therefore, people of differing cultural backgrounds may have different ideas about their identities and how they should act in this world. Identity then is a culturally constructed belief system: the combination of cultural principles and environmental and situational input create what we see as identity; identity, and the self, is a set of beliefs built from experiences that inform our actions and our perceptions.

\subsubsection{How Is Identity Constructed?}

Identity is a social construct. Our self-representation, our identity, guides us through our personal reality, which has been constructed simultaneously by our experiences and our cultural principles. This internalized theory of the world, this set of meanings, will guide a person when they occupy a role in society, when they are a member of a group or when they call on certain characteristics to distinguish or dissociate themself from other individuals or groups (Burke \& Stets, 2009). This theory of the world is built through social interactions, which begin from the time we first partake in exchanges with others.

\subsubsection{Identity and Early Socialization}

The theory of attachment bonds, as first put forth by John Bowlby $(1969,1973)$ and further researched by Mary Ainsworth $(1969,1979)$, addresses the behaviours of children with their primary caregivers. Van IJzenjoorn and Sgi-Schwartz (2008 as cited in Renick Thomson, Kennedy, and Kuebli, 2011) summarize the central hypotheses of this theory as follows: attachment is universal: all children become attached to one caregiver over others (the exception is infants with neurophysiological impairments, as underlined by Renick Thomson, Kennedy, and Kuebli, 2011); attachment security is normative; attachment security depends on sensitive and competent caregiving; attachment security 
may predict "differences in competencies, such as emotion regulation" (Renick Thomson, Kennedy \& Kuebli, 2011). Over the years, four types of attachment bonds have been agreed upon: secure, avoidant, ambivalent or resistant, and disorganized/disoriented (Howe, 2011; Renick Thomson, Kennedy \& Kuebli, 2011).

All children (save some extreme exceptions) form attachment bonds with their caregivers, including deaf children (Renick Thomson, Kennedy \& Kuebli, 2011). Hearing children who are exposed to their mothers' voices prenatally have been found to show a preference for the mother's voice postnatally (Renick Thomson, Kennedy \& Kuebli, 2011). This is believed to play an important role in the formation of attachment bonds between mother and child. Deaf infants are then at a disadvantage in this regard, as compared with their hearing peers. Despite the challenges associated with hearing parents raising a deaf child, these children have been found to behave in ways that compensate for their differences (through the use of tactile and visual contact, for example) (Renick Thomson, Kennedy \& Kuebli, 2011).

The development of identity seems to be ontogenetic process. According to Foddy and Kashima (2002) the interactions that occur between biological maturation and socialization experiences lead an individual to form a sense of self. The environment in which the child spends their formative years determines what developmental changes take place within their mind as the attachment bond takes place. Of special importance here is the presence and interaction that takes place between the child and their primary caregiver, which plays a central part in the child's social development and the construction of identity. The quality of the child's attachment to their caregiver has the potential to instill in the child, feelings of security that are necessary for them to explore 
the world and attempt to form relationships with others (Weiss, 1998); the quality of the attachment, then, acts as "the foundation for the quality of the child's other social relationships" (Renick Thomson, Kennedy \& Kuebli, 2011). Despite the apparently crucial role of attachment bonds, their long-term effects are not agreed upon and "when significant associations are found they tend to be modest" (Renick Thomson, Kennedy \& Kuebli, 2011, p. 32).

Nonetheless, according to Yoshinaga-Itano (2011), "optimal and successful communication interactions" (p. 88) between deaf children and their parents are of utmost importance. As well, parents' stress levels seem to have an effect on deaf children's development (Hintermair, 2006). As Foddy and Kashima (2002) explain, the relationship with the primary caregiver shapes the child's internal standards and values. These standards and values are later used as a comparative tool for judgments made about the self; they are also used to measure levels of esteem and further, they dictate how the individual will approach others.

\subsubsection{Friendship and Group Membership}

As opposed to relationships with family members, friendship is a unique kind of relationship because it tends to be voluntary (Weiss, 1998; Tillmann-Healy, 2006). Friendship is an important part of identity construction as "conceptions of self and other are formed, reinforced, and altered in the context of ongoing relationships" (TillmannHealy, 2006, p. 275, emphasis mine). As Weiss (1998) proposes,

Each participant in a relationship is affected emotionally and cognitively by its content. Some relational events contribute to participants' functioning or wellbeing; they heighten participants' self-esteem, reassure participants of the other's support, or provide participants with information and direction... But relational effects can also constrain, burden, or injure participants". (p. 674) 
Friendships are initially formed on the basis of similarities in attitude and values because these commonalities provide a kind of self-validation and this makes a relationship rewarding. Further, friendship selection appears to be based on visible characteristics, like behaviors and activities, which exemplify values (Urberg, Degirmencioglu and Tolson, 1998). Initially then, an individual may assume at a subconscious level that another person possesses similar values, as shown through behaviors, which the individual associates to these values.

When an individual shares the beliefs and values of a certain culture, they may choose to display these sentiments through group membership. In other words, group membership is an outward expression of the intention to subscribe to a certain culture and its norms. Members of a group share a system of meanings that they experience as their realities; the system is also known as a social order. Within the social order is a collection of categorized objects, people and events. This classification gives members of a group a sense of safety and understanding about the world (Jones, Farina, Hastorf, Markus, Miller, Scott, \& French, 1984).

Woodward (2004) explains, "Identity provides a link between individuals and the world in which they live. Identity combines how I see myself and how others see me. Identity involves the internal and the subjective, and the external" (p. 7). But given that many of us belong to more than one social group, our self-representation tends to alter slightly based on our social surroundings. One individual, then, will internalize a variety of group identities, each one appropriate to a specific set of interpersonal relationships.

A member of the majority population will have access to a wide range of groups to choose from. Members of minority groups, on the other hand, may not always have the 
same freedoms when it comes to the creation, maintenance and dissolution of friendships, as do members of the majority group (Makabe, 2005). This is because of the sheer number of people within those minority groups and it extends to their potential for joining a variety of groups as well.

For deaf children who are surrounded by hearing peers, forming friendships may not be as straightforward as for hearing children. When deaf children are placed in a mainstream school, they are often the only deaf child in their class, if not in the whole school. Being different from the norm may impact their abilities to form friendships in this environment because the similarities they might share with other children are not immediately apparent, nor are they easily communicated. Interruptions in friendships, or the formations thereof, may prevent deaf children from finding links between themselves and the world in which they live, and therefore, may affect the way their identities are constructed.

\subsubsection{Language Ideologies, Minority Languages, and Identity}

As was explained above, language plays an important role in the construction of identity. From very early on, the linguistic and semiotic interactions we have with others form into our identities. Language and identity are fundamentally entangled, in part because language is a uniquely 'human' characteristic (Edwards, 2009). Language is also the means by which we express our social and cultural affiliations, in short, our identities. On the other hand, the languages we use to express such loyalties carry social currency, and the assumptions we, and others, make about our languages can also have an effect on our identities. Bourdieu (1991) put forth the idea of linguistic habitus: "a set of dispositions which incline agents to act and react in certain ways" towards languages 
(Thompson, in Bourdieu, 1991, p. 12). These dispositions and attitudes are learned from very early socializing, onward, and are formed from exposure to language attitudes displayed by adults and the greater social environment (including schools) and they are "a group or class phenomenon" (Ó Riagáin, 2008, p. 336).

Language attitudes formed within individuals “...[reflect] social perceptions of the speakers of given varieties and [have] nothing to say about any intrinsic qualities logical or aesthetic - of the language or dialect itself" (Edwards, 1999, p. 102). In fact, as Blackledge and Pavlenko (2002, as cited in Ó Riagáin, 2008) explain,

Language ideologies are thus about more than individual speakers' attitudes to their languages, or speakers using languages in particular ways. Rather, they include the values, practices and beliefs associated with language use by speakers, and the discourse which constructs values and beliefs at state, institutional, national and global levels. (p. 336)

Linguistic habitus and language attitudes are both apparent and acted upon at the individual level, group level and societal level. At the societal level, minority language policy decisions reflect society's attitudes and perceptions towards those minority languages, and these important decisions impact those minorities' identities (Freeman Field, 2008). When language policies that limit the uses of minority languages are put in place, members of a society may internalize the negative tone of these decisions. As Edwards (1999) explains, "perceptions [...] operate in societies, most of which are stratified in various ways and which - to put things bluntly - power and status are often able to translate social difference into social deficiency" (p. 101). In other words, minority language policies have a salient effect on language attitudes by qualifying difference, turning it into limitation. 
Ironically, then, minority languages that are viewed by their users as markers of status within those groups are often viewed by greater society as something contrary to that. As Ó Riagáin (2008) puts it, "a minority language that is valued for its identity and solidarity functions may simultaneously be seen, even by its own speakers, as weakly endowed in terms of status" (p. 330). American Sign Language is a minority language that suffers from this type of oppression and which, consequently, is stigmatized to the extent that mainstream society assigns it little to no prestige ${ }^{2}$. The status of American Sign Language as a language of instruction will be examined in some detail at the bottom of section 3 .

\subsubsection{Multiple and Contradictory Identities}

Identity has been suggested to be a multiplex construct: rather than individuals having one static identity, people are instead made up of discrete identities based on context, community and surroundings. Pittam (1999) explains,

Who we are, our personal and social [identities], [are] fundamental to all social interaction. [...] That they have an impact on social interaction is perhaps uncontroversial. Whether we can simply accept them as stable, a priori categories is another matter, however. (p. 114)

That is, as we identify with a variety of groups, so too, our social self must adjust to fit different environments and the cultural demands of groups, which are unique to each group. Our identities are "socially recognized [positions], recognized by others, not just by [ourselves]" (Woodward, 2004, p. 6) and are negotiated in the contexts of situations. For this reason, individuals are made up of a collection of selves, appropriate to different frames of reference. In particular, research on additional language learners

${ }^{2}$ See Reagan (2011) for a discussion of the limited, though important, advances made in the rights of ASL. 
and identity shows identity to be "dynamic, contradictory, and constantly changing across time and place (Norton, 2008, p. 47).

People do not to tend to act in the same manner all the time and in fact, Pittam (1999) even claims that they "can and do hold apparently contradictory positions vis-àvis their own identity even across the space of a single interaction" (p. 116). Further, Woodward (2004) holds,

The link between myself and others is not only indicated by the connection between how I see myself and how other people see me, but also by the connection between what I want to be and the influences, pressures and opportunities which are available. Material, social and physical constraints prevent us from successfully presenting ourselves in some identity positionsconstraints which include the perceptions of others. (p. 7)

So, the construction of identity is collaborative, but comprises cultural knowledge that a person accumulates over time. It also changes as the situation requires and may even be contradictory in any given instance. Contradictions occur in the presence of conflicting values based on sets of norms associated to different groups. When people find themselves to be different than the majority, their distinguishing characteristics play a role in the construction of their identities as well.

\subsection{Deaf Identities}

The concept of deafness tends to be interpreted in one of two ways. One focuses on the clinical-pathological aspects of the word (Baker \& Cokely, 1980), while the other emphasizes a cultural connection between word and concept. These definitions carry opposing value judgments; the clinical-pathological definition of deafness is based on a hearing-centric view of normality, which is largely based on the medical profession's attitudes towards deafness. The cultural definition is one based on alternative ideas of 
normality: the cultural view of deafness challenges the idea that deafness is a disability, an assumption that is held by the hearing majority (Reagan, 2010).

\subsubsection{Deafness and Disability}

The clinical-pathological definition of deafness - where deaf is spelled with a lower case ' $d$ ' - views hearing loss as a deficit. Principally, this word is used to designate the condition of lacking function of the auditory sense. The clinical-pathological definition of deafness does not take into account any of the social aspects of deafness, which will be treated below; here, the focus is on flaw, difference and weakness. The medical community is most prominently associated with this perspective, but many schools operate under this principle as well. The condition of deafness is something to be repaired or dealt with technologically, through the use of hearing devices. Many promoters of this definition emphasize the need for deaf children to learn to speak and hear while others who also support this notion of deafness, reject this idea, maintaining it to be misguided, and instead underline the need for deaf children to learn to read and write.

Disability is a socially and culturally constructed concept: some human variations and differences are accepted, while others are not. Lane (2002) notes, for example, that variations in height are generally accepted but very short people are considered disabled. Disability then is an attribute that leads to restricted functioning and allows for the technologies of normalization to come up with a treatment. This medicalization shifts the responsibility for social inequality onto the individual, who is now considered to have a treatable condition (Lane, 2002.). 
The clinical-pathological view of deafness imposes on deaf people the expectation that their lives will be a struggle and that they are incomplete compared with the majority. The tendency for deaf children is for these children to see themselves as normal, and not to dwell on, or be concerned about, their inability to hear (Baker, 1999). Deaf children are not born with the knowledge that their bodies are lacking. It is not until a deaf child has been exposed to the hearing majority's conceptions of deafness that they become aware of the perceived problems associated with their situation. They may eventually learn that the dominant groups often view signing as strange, different, inferior to speech, and as a mark of deficiency (Baker, 1999). They will learn from those around them that they are considered disabled or handicapped.

In 1990, the Americans with Disabilities Act was passed in the United States; Canada has no equivalent federal bill, however, in 2005 the Accessibility for Ontarians with Disabilities Act was passed (Accessibility for Ontarians with Disabilities Act, 2005). Deaf people were often handicapped by the dominant culture's so-called unintentional forms of discriminations, such as inaccessibility to telephone systems, the television and media industry, and communication barriers that prevented them from accessing public programs and accommodations. Technology is often in an attempt to fix this discrepancy, but despite advances made in the field of hearing devices, "no medical solution as yet has provided full access to spoken languages equivalent to a functioning ear" (Rayman, 2009, p. 340). The harsh irony is that in accepting accommodations offered under the umbrella of disability rights, deaf people must concede that they are disabled. By taking advantage of what is rightfully theirs - access to information, the opportunity to partake in larger 
society - they undercut their own claims of equality. In addition to this, they also reinforce the stigma associated with deafness.

\subsubsection{Stigma}

Deaf people are considered disabled due to stigmas attached to deafness. While it is true that deafness sometimes accompanies other debilitating conditions, deafness itself, does not make a person incapable of learning, as some cognitive disabilities may, nor does it affect a person's physical abilities, such as their motor skills. Rather, deafness causes a disadvantage to the afflicted because of what modern society considers the norm. Society is made up of positions that are related to one another in some way (Burke \& Stets, 2009), and the relations between these positions are what account for classes. Certain positions in society are favored while others are less favored; stigma is attached to some less favored positions while preference is associated to those that are favored. Conditions that render a person challenged to perform typical tasks differently are stigmatized. Stigma occurs in response to some mark that sets a person apart from the general population and deafness marks a person in this way. Jones et al. (1984) present six factors that affect stigmatization:

1. Concealability. Is the condition hidden or obvious? To what extent is its visibility controllable?

2. Course. What pattern of change over time is usually shown by the condition? What is its ultimate outcome?

3. Disruptiveness. Does it block or hamper interaction and communication?

4. Aesthetic qualities. To what extent does the mark make the possessor repellent, ugly, or upsetting?

5. Origin. Under what circumstances did the condition originate? Was anyone responsible for it and what was he or she trying to do?

6. Peril. What kind of danger is posed by the mark and how imminent and serious is it? 
Of these factors, concealability seems to be of utmost importance, as it can be seen to feed the subsequent constituents; if the concealability of a mark is great, the other factors tend to be less significant. Deafness is initially concealable, so long as the deaf person does not try to communicate. The power of concealability is so potent, in fact, that Jones et al. (1984) report that if marked persons can conceal their differences, this will diminish the effects of disruptiveness; if they cannot conceal their difference, disruptiveness will be compounded. For a deaf person, whose speech tends not to resemble that of a hearing person's, disruptiveness may be a more important issue than Jones et al. account for. Moreover, the effects of stigma may be felt to varying degrees.

\subsubsection{Bullying}

One of the results of stigma is the possibility of bullying. Jolliffe and Farrington (2010) define bullying as "a systematic abuse of power" (p. 2, in Smith \& Sharp, 1994), which "involves repeated incidents between the same dyad of bully and victim over prolonged periods of time" (p. 2) and an imbalance of power (where the bully is more powerful and therefore can oppress the other).

The stigma connected to deafness often translates into an assumption of inferiority, and bullies often target deaf children (Bauman \& Pero, 2010). In fact, Bauman and Pero (2010) report that children with "observable disabilities... are twice as likely to be bullied as children whose disabilities are not readily apparent" (p. 236). Deaf children report being bullied by their hearing peers, specifically with regards to their communicative abilities and vocal characteristics (Weiner \& Miller, 2006).

Victims of bullying may react to acts of physical, verbal or mental aggression by blaming themselves for their peers' behaviours. Self-blaming attribution (Bauman \& 
Pero, 2010) is a process by which the victim takes ownership of the responsibility for the bully's actions. The victim may account for the abuse by putting the onus on "their own deficits, rather than [on] an external cause" (Bauman \& Pero, 2010, p. 240). Since deafness is a condition that cannot easily be overcome or changed, the reaction of selfblame may result in a feeling of powerlessness to stop the bullying, or an expectation that it will continue indefinitely. Self-blaming attributions cause victims of bullying emotional distress (Bauman \& Pero, 2010).

When people are bullied due to stigma, they may feel the need to change that aspect of themselves that marks them as different. Technology provides a means to changing one's situation. But technology is not the only way for a deaf person to find their footing in the world. In order to become a member of a group, a deaf person can also improve their communication skills.

\subsubsection{Transformation}

According to McCracken (2008), perceived status locations that are fixed along a vertical continuum in social space, as conceived of in Western societies, may result in attempts by individuals to transform. Transformation occurs when an individual tends to certain aspects of their being, internal or external, in order to join a better-situated group. According to this understanding of status, 'upwardness' is deemed favorable, and therefore, upward movement is desirable.

One of the key tasks in any upward transformation is the perfecting of conversational skills. McCracken (2008) contends that these must be upheld since "conversation offers the best opportunity to detect pretenders" (p. 56). This notion is connected to Jones et al.'s (1984) disruptiveness factor. When a person's speech is 
different - be it an accent, impediment or some mixture of the two - that person may be stigmatized and may seek to change their speech to fit the norm's. And in the act of modifying their language, they modify their person by learning to express themselves differently and with different people in different social situations.

Transformation allows humans to come to terms with, and respond to, the fact that the human character is fluctuating and inconstant - one of the most daunting aspects of the human condition (McCracken, 2008). McCracken (2008) further explains,

We are, most of us, the products of a static, continuous world. We expect continuity, sameness, and consistency. We think of 'identity' as something that comes from things that remain 'identical' - from continuities of self, family, home, and work. But we could be wrong. Perhaps it comes from transformation. $(\mathrm{xx})$

As such, communication and language are things upon which identity is contingent. Identity, when it is a result of transformation, is also dependent on other tools and contexts that have transformational powers, such as technology and community. Indeed, through transformation, identities are fluctuating, ever-adjusting aspects that alter for social situations, such as family, home and work. Identity can then change by transforming one's auditory context through technology or by transforming one's social context through group membership and community.

Technology plays an important role in the "improvement" of deaf people.

Technology and transformation in this direction, however, is aimed at the assimilation of deaf people into mainstream society, as evidenced by the association between cochlear implants and spoken language (Wheeler et. al., 2007). The preference for deaf children to learn to speak, rather than learn language in general - signed or spoken - is (sometimes unintentionally) articulated in much of the literature treating issues of hearing 
technologies (Gravel \& O'Gara, 2003; Hyde \& Punch, 2011; Niparko \& Blankenhorn, 2003; Renick Thomson, Kennedy \& Kuebli, 2011). Although hearing aids are a transformative tool, they are not the same thing as cochlear implants in that their effects are not necessarily permanent. The act of wearing a hearing aid does not entail rigorous auditory-verbal therapy, as does implantation of a CI, nor does the act of wearing a hearing aid entail that the wearer in being committed to assimilating into the hearing majority.

As may have become apparent by now, the issue of deaf identity is not exactly a binary one: rather than the decision being about whether or not one identifies as hard of hearing, for example, the idea of identity among the deaf population is better gauged on a continuum (Reagan, 2005).

\subsubsection{Cultural Deafness}

Up to now, I have presented deafness as conceived of through two related lenses. The first description of deafness was a purely physiological and medical explanation of how deafness affects a person, as well as other related themes, such as language and education, theoretical conceptions of identity, socialization and group membership. Technology, and its implications, has also been discussed. The following discussion of deafness as a group identity encompasses all the above themes.

Despite the barriers that deafness can cause, people who are deaf do not necessarily focus on the negative aspects of their situations. As Reagan (2010) points out, hearing people tend to "project their concerns and subtractive perspective onto Deaf people" (italics mine, p. 10), but members of the Deaf community feel contrary to this. The cultural definition of Deafness - indicated by the use of a capital letter ' $D$ ' - focuses 
on the history of deaf people, their plights, their values and goals. Most importantly, Deaf people share a common love of their language, Sign Language. Those who identify as Deaf also tend to behave in distinctively "Deaf" ways; there are rules dictating eye contact patterns and physical contact norms (Reagan, 1995). Finally, Deaf people do not see their auditory context as an impediment or a handicap. They do not see it as something that needs to be fixed or altered (Carty, 1994), and it tends not to be a characteristic of members of the Deaf community, that they wish to make use of technology to alter their auditory states (Reagan, 1995).

Not all persons who do not hear choose to associate with the Deaf community; likewise, there is (in theory) no minimum qualifying degree of hearing loss required to join the community (although it is easier to join when your hearing status connects you to the group). However, Carty (1994) explains that Deaf identity has certain defining characteristics, such as the acceptance of deafness as a central and positive part of one's being, therefore someone with mild hearing loss could still self-identify as Deaf. Another crucial characteristic of Deafness is linguistic competence in ASL (Kannapell, 1994).

Residential schools for the Deaf are an integral part of Deaf culture because, unlike other cultural groups, the Deaf community does not have its own native territory (Lane, Hoffmeister \& Bahan, 1996). These schools, then, act as surrogates for this absence, providing Deaf people with a physical "land" to call their own. Here, participation in sports is encouraged and nurtured, and team sports are also a central aspect of Deaf culture. Sports are important for Deaf people because they are often a means by which new students arriving at residential schools acculturate themselves in this new environment. Additionally, when involved in competitions with other schools, 
sports provides Deaf students with the opportunity to be seen as equals; their oral language skills are not scrutinized in this setting (Lane, Hoffmeister \& Bahan, 1996).

Indeed, in these environments, stigma exists differently. As Lane (1992) explains, stigma is relative: "In the [D]eaf community, to be called 'oral' $[\ldots]$ is unacceptable. 'Oral' means you have made the wrong life choices, you have uncritically embraced alien values that place a premium on speech. Hearing people fail to see what is wrong with deaf people's being 'oral'; articulateness is prized in American society; gesturing is not" (p. 7). Deaf people connect with one another on this important level and Schools for the Deaf provide a forum for equality and an alternate identity.

Members of the Deaf community also identify with one another through their experiences with oppression. Deaf people face many challenges in society and many of these are considered inadvertent discrimination, by which they feel oppressed. Many professions intended to facilitate deaf people's lives are held by hearing individuals, and in these roles, hearing people hold a position of power that can sometimes result in oppression. This is not something that has gone unnoticed by those involved, and some are working to remedy the situation, however, the imbalance remains (Lane, Hoffmeister \& Bahan, 1996).

Carty (1994) suggests guidelines for the process involved in the selfdetermination of one's Deaf identity. The first step is the realization by the person that they are different compared with the general population and becomes confused at this discovery. Next, they feel frustration, anger or blame, which they may or may not express (internalization may result in self-hate, while expressions may manifest in outbursts or non-conformity). The following step is exploration, where the individual will 
explore identity options. This step can be shaped by the availability, or unavailability, of information pertaining to Deafness. Step four entails either identification with or rejection of the Deaf community, but is likely a premature decision on both counts. Ambivalence may follow, especially if, for example, identification with the Deaf community allowed for some unpleasant experiences. Finally, acceptance comes when one has had sufficient experience and gained enough information to make an enlightened decision about their identity.

\subsection{6 "Small-d deaf" Identities}

A deaf person may not choose to identify with the Deaf community. If a deaf person chooses not to see their hearing loss as something positive, and chooses instead to do what they can to work with their residual hearing, they may be called hard-of-hearing. Hard-of-hearing people, defined as those people whose hearing does not match the majority's but who nonetheless make use of an aural-oral language as their primary mode of communication, are members of society who do not ascribe to Deaf culture, its values, and beliefs, and who prefer not to be visible for their difference (Sorkin, 1996). Indeed, they strive to be 'normal'; to blend into the mainstream as any hearing person does, albeit with the help of assistive devices (Sorkin, 1996). In fact, technology seems to play a prominent role in decision-making here. As Wald and Knutson (2000) found when interviewing a group of 45 adolescents with and without cochlear implants, those who made use of the technology had a generally more positive view of the idea of hearing identity as a goal.

Sorkin (1996) points out that the Deaf community makes up about two percent of the population of hearing impaired persons in the United States. This leaves a large group 
of people who prioritize membership in the hearing world, although their auditory histories may differ in important ways. Within this group are late-deafened individuals, others who have experienced gradual onset deafness, and others still who are prelingually deaf but attempted (or were made to attempt) to integrate into larger society.

Ross (1993) - an audiologist - holds that there must be a distinction made between Deaf and hard-of-hearing persons from a cultural point of view. He argues that these persons have different personal and social identities but also that they have different accommodation needs. He implies that the self-label Deaf is one that is chosen out of necessity and that some people who identify as Deaf in fact are hard-of-hearing, and were not properly cared-for in childhood. That is, whatever residual hearing they had was not cultivated and therefore, these children grew up unable to live life as a hard-of-hearing person, ultimately being forced into the Deaf community. He explains that those hard-ofhearing children who grow up to join the Deaf community are those who suffered the most in elementary school: they were isolated, excluded and felt separate from their "normal hearing peers" (Ross, 1993). This attitude - normal versus deficient - represents perfectly the general opinion of the medical community.

Hard-of-hearing individuals, as Ross (1993) points out, are not a visible minority. For this reason, and because they are so often pressured to acculturate with the majority group, they tend not to form communities, or even regroup on a smaller scale.

Interestingly, there has also been a distinction of the term hearing that mirrors the D/deaf contrast. Napier (2002) proposes that, as the capital letter $D$ in Deaf designates a person who subscribes to a set of beliefs and values, so too, a capitalized $H$ for Hearing can be used to refer to a non-deaf person who subscribes to the majority beliefs and 
values and who accepts the clinical-pathological definition of deafness. Further, a Hearing person is someone who may refute claims of a Deaf culture and who sees deaf people as disabled and as subordinates. In other words, the capitalization of the initial letter renders the term a culture-affiliation designation, while the typical spelling can more simply refer to a physical context. So, a hearing person is someone who is not deaf but who does not subscribe to elitist Hearing conceptions.

This distinction in terms serves that group of hearing people who are staunch supporters of Deafness, giving them a special classification that sets them apart from the majority of uninformed "Hearing" people. Yet to group all "Hearing" people as such is too simplistic and frankly, gives the false idea that the issue is black and white. Indeed the issue is far more complex than Napier concludes, as many people do not wish to support either side wholly. Gesser (2007) explains that in the case of hard-of-hearing individuals, there is often a feeling of belonging to neither group (Hearing, hearing or Deaf), a situation of "between-ness". This too can be felt by those superficially included in the hearing grouping. Such feelings of uncertainty about one's identity are also characteristic of deaf people who make use of cochlear implants. In their study Cochlear Implants: The Young People's Perspective, Wheeler, Archbold, Gregory and Skipp (2007) reported finding that there seemed to be "some ambiguity as to whether [the participants] think of themselves as deaf or hearing" (p. 311). Furthermore, none of their participants claimed to be members of the Deaf community, or to subscribe to Deaf culture. 


\subsubsection{Deaf Identity, Sign Language and Language Ideologies}

Language plays an important part in the creation of identity. Firstly, it is, as Edwards (2009) points out, "central to the human condition, and [...] the most salient distinguishing characteristic of our species" (p. 20). Further, language is a group-identity marker: "accent, dialect and language variations [...] reveal speakers' memberships in particular speech communities, social classes, ethnic and national groups (Edwards, 2009, p. 21). Identities are made apparent by language use, then, and as such, the general public's opinion of languages, dialects and accents becomes an issue. If we assume, as explained previously, that identity is formed in a process that integrates others' with our own views of ourselves, then the ideologies associated with the languages we use is an important element in the construction of identity.

Reagan (2011) defines language ideology as "beliefs and attitudes that are specifically concerned with language and linguistic issues" (p. 608). Language ideology is an omnipresent concept that plays an important part in society, social relations as well as in education (Reagan, 2011).

When a state does not grant its citizens the right to their natural mother tongue in this case, deaf people the right to Sign Language - "this lack of rights is what often leads to and/or can be used to mobilisation [sic] of sentiments which can then be labelled [sic] "ethnic conflicts"' (Skutnabb-Kangas, 2001, p. 204). At the individual level, the lack of rights may be internalized and may manifest as discrimination. In other words, even if mobilization does not occur, the intentions behind legislation can be felt by the population, and the result may be a sense of disdain for that language. 
In terms of Sign Language, ideology has an effect in two ways: first, deaf children are exposed to English (or the spoken language dominant in the area) in a unifying effort. That is, presumably, spoken language acquisition is the key to assimilation. This leads to the prevention of learning Sign Language. Interestingly, even though much has been published about the appropriateness of Sign Language as the deaf child's natural first language, it remains something that is avoided at all costs. Deaf children have a difficult time learning spoken language as their first language, but are often encouraged to learn the dominant spoken language rather than their natural language, Sign Language (Grosjean, 2010).

When it comes to the Deaf community, ASL (or whatever the dominant Sign Language of the region is) is the most important element of the culture and the identity. ASL functions "not only as the community"s vernacular language, but also as an indicator of cultural group membership" (Reagan, 2010, p. 11). Member of the Deaf community presumably use ASL to mark their group membership and a high level of prestige is associated with ASL within the community. Other types of visual language are used by Deaf people but in these instances, Deaf people are not necessarily communicating with other Deaf people. For example, contact Sign Language (Pidgin Sign Language) is often used between Deaf people and hearing people, or non-native signers (Reagan, 2010). Indeed, Reagan (2010) claims that there is an important emphasis put on the type of signing that takes place between people who are Deaf: signing must be through ASL in order to be a marker of group identity.

Although ASL is a marker of status within the Deaf community, it is far from being one outside the community. Reagan (2011) reports on significant strides made in 
the official recognition of ASL in the United States, but underlines that all the advances made in this area are "fundamentally based on the deficit views of ASL" (p. 611). And while some states have passed legislation that recognizes ASL as the official and native language of Deaf people in those states, the fact that this needs to appear as legislation speaks loudly to the work that still needs to be done in this area.

The lack of recognition of ASL as a natural language affects Deaf identity in two ways: first, by refusing to acknowledge the legitimacy of ASL, and therefore keeping it from deaf children, the population of native Deaf signers is extremely limited; second, by omitting to legitimize the language, and instead promoting its status as the right of an inferior and disabled minority, ASL is propagated as the language of handicapped people. The former issue can be seen to have an effect on Reagan's above claim, that Deaf people use ASL among themselves, within the community. This, however, is not as consequential as the latter effect, as this one leads to stigma. By disallowing ASL and its first-language users to be seen as equals, pressure to identify with the hearing majority increases.

Linguistically, deaf people may be faced with choosing to identify as bilinguals or multilinguals. Whereas bilingualism was once believed to diminish cognitive abilities, it is now seen in an opposite way: "some have argued that increases in linguistic repertoire correlate with heightened sensitivity, enhanced cultural awareness, perhaps even greater cognitive flexibility and all-round nous" (Edwards, 2009, p. 248). What is problematic in this situation, though, is that many deaf people do not develop into fully functioning bilinguals. In fact, because of their often-limited or erroneous ASL input, they tend to 
struggle to learn English properly too. The result is, as Reagan (2011) puts it, that deaf children are to some extent alingual.

\subsubsection{Deafness, Language and Education}

Each of the above definitions corresponds with one of the two main schools of thought pertaining to Deaf education and the best way to instruct deaf pupils. The point of contention in debates on how to educate deaf children hinges on the language of instruction. The majority of deaf children are made to adopt the dominant spoken language as their own, and many are not exposed to visual language until late in life, if at all. However, there is a relationship between ASL proficiency and English literacy skills (Freel, Clark, Anderson, Gilbert, Musyoka \& Hauser, 2011; Gibson, Small \& Mason, 1997; Myers, Clark, Musyoka, Anderson, Gilbert, Agyen \& Hauser, 2010) and it is important that a deaf child be taught a visual language within the first few years of life (Grosjean, 2001) in order to facilitate their acquisition of literacy skills.

The language of education of deaf children is an important part of the issues revolving around deaf identity. School should leave individuals with a collection of knowledge greater than when they entered the system, but it should also provide a social arena, where the child may learn how to interact in the world. Socializing for deaf children is facilitated by the use of visual language (Grosjean, 2001). Mainstream schools, however, do not make use of Sign Language very often, if at all. In situations where children may not build relationships through communication and interaction, they may not be able to build a solid identity. Sign Language in school would offer deaf children a better opportunity for socializing. Unfortunately, the importance of socializing 
in school is often ignored, and intellectual intelligence takes precedence over emotional and communicative intelligence.

Oralism (associated with the clinical-pathological view of deafness) is a method of educating that focuses on spoken language as the medium for instruction while manualism (a constituent of the cultural definition of Deafness) is a method that endorses the use of visual language to teach deaf children. Both these methods emerged in Europe in the late 1700 s. Paris is home to the world's first government-sponsored school for the deaf, where Sign Language was first used as an instructional medium (the school is still open today). Soon after, the first school for the deaf that was opened with the intention of teaching them spoken language was established in Leipzig (Lang, 2003). Although the founder of the school in France, Charles Michel Abbé de l'Epée, saw Sign Language as a natural mode of communication for deaf people, he nonetheless combined the signs he had learned to his own invented grammatical system, which diverged considerably from the "natural" language his deaf pupils were already used to (Lang, 2003). Meanwhile in Germany, Samuel Heinicke, founder of the first oralist school, employed the use of speech to teach the students at his school.

Though the early attempts at educating deaf children were far from perfect, what became apparent from these beginnings was that deaf pupils could indeed learn to read and write (especially through visual language). The first school for the deaf in North America was founded in 1817 in Hartford, Connecticut. Thomas Hopkins Gallaudet, a scholar whose neighbor had a deaf daughter, traveled to Europe to study the methods used to teach deaf children. His quest took him first to England and then to France, where he met Laurent Clerc, a deaf assistant teacher at the Paris school, whom he convinced to 
come to America. Together, they opened the Connecticut Asylum for the Deaf and Dumb (today named the American School for the Deaf). The opening of the school set off a chain reaction of sorts, and by 1850 more than 15 residential schools for the deaf had been established in the United States. What's more, almost $40 \%$ of the teaching staff at these schools were deaf themselves (Lang, 2003). The result of this wave meant that deaf employment grew, $\mathrm{D} /$ deaf people had access to better jobs and the Deaf community grew significantly.

This was a very positive time for the community, even being referred to as the "Golden Age" for the Deaf community (Gibson, Small \& Mason, 1997). Many talented deaf people started to gain attention; they were becoming scientists, artists, and writers, among other things. Demand for higher education became a bigger focus and before the turn of the century, the Deaf community had a college of their own (presently called Gallaudet University, founded by T.H. Gallaudet's son, Edward Miner Gallaudet).

Meanwhile, the oral-manual debate continued and one of the more prominent figures in support of the oral method was Alexander Graham Bell. Bell came from a family very much involved in deaf education and deafness: his father was an educator of deaf children and his mother was deaf. He believed that Sign Language was not a language at all, but was in fact a vernacular that made assimilating into wider society very difficult for deaf people. His goal was for them to be resourceful, independent and productive in the hearing world. As oralism became increasingly popular in the United States, hearing women started to be trained to teach deaf children in mainstream schools. By 1910, the greater part of Deaf schools had banned the use of Sign Language in the classroom (Greenwald, 2007). 
Bell feared that isolation from the hearing majority, and residential schools for the Deaf where signing was allowed or encouraged, would result in a greater chance of deaf people procreating. This, he worried, would lead to a "deaf variety of the human race" (Greenwald, 2007). He aligned himself with the American eugenics movement (and Charles Benedict Davenport, a prominent proponent of eugenics) and although he believed that imposing legislative restrictions on marriage was a violation of one's liberty of free choice, he actively discouraged deaf pupils from seeking mates within the deaf community. Despite this affiliation, elite members of the Deaf community focused their objections on Bell's efforts to eradicate Sign Language (Greenwald, 2007).

The first half of the twentieth century was a fertile time for the education of D/deaf people; although it seemed oralism had won the debate, many advancements nonetheless occurred. This moment in history saw the beginning of special education: educators of deaf children were publishing arguments for new and practical ways of reaching these children's cognitive faculties. Deaf children were also being placed in special classrooms within schools attended by hearing children (Lang, 2003).

Finally, in 1960, William Stokoe, an English professor at Gallaudet University, presented research wherein he showed ASL to be a true and natural language. While this claim was not uniformly accepted by the Deaf community, it did serve to raise awareness and soon Deaf people were taking a political and social stand (Lang, 2003). This event, the naming of ASL as a real language, played an important role in the evolution of how the Deaf community saw itself. With a language now recognized by scholars to be a true language, Deaf people were able to take some pride in ASL. 
In terms of educational advancements, however, Stokoe's research has not had a consistent effect. In the past 50 years, a number of laws have been passed in the United States dictating the course of special education. Today, in Ontario, there are two options available to a deaf child. The child may attend a residential school for the Deaf, where bilingual bicultural education is the aim (a mixture of visual and auditory languages are used in the schools today). The other option is for the deaf child to attend a mainstream school where the amount of time spent in a hearing classroom varies according to the needs of the individual child.

Despite advances in the status of ASL, and developments to the educational systems available to deaf children, education remains a point of contention among those affiliated with deafness in one way or another (the medical community or members of the families of deaf children, for example). In Schools for the Deaf, where Sign Language is used, deaf children are surrounded by peers who share physiological similarities. Gibson, Small and Mason (1997) explain that bilingual bicultural education is education that empowers the deaf child; it is "one that fosters a sense of pride and self motivation in Deaf individuals" (p. 231). In this context, deaf children may build world knowledge and friendships that lead to clear identities. When deaf children are put into the mainstream, where Sign Language is not used and their peers are physiologically vastly different from them, deaf children may be isolated and lack an understanding of the world, since they are effectively being shaped to function as "semi-hearing" people (Gibson, Small \& Mason, 1997). This would have an effect on the way in which their identities are formed. 


\subsection{Summary}

In this chapter, the scope of this study was explained, and the central concepts were defined. I began by defining deafness in medical terms, and by giving a survey of the technological advances made in the medicalization of deafness, and the related solutions.

I then presented the topic of identity both from a psychological and a social perspective. Identity was shown to be a multiplex and nuanced process; that it is progressive and emergent; and that it is cultivated through social interactions and relationships. Throughout this section, it was underlined that deaf people may struggle with certain aspects of interaction and human relations.

In the final section of this chapter, an explanation of the social aspects of deafness was given. This one brought to attention the various conceptions of deafness: deafness as disability, Deaf culture and how language and education come into play. Importantly, the idea of grappling with the notions of 'deafness as disability' and 'deafness as community' was discussed, as was the issue of transformation. Transformation was shown to potentially take place by migrating to another community, or by adopting the use of technology to make one more 'hearing'. By these parameters, it was established that the construction of identity of deaf people is a complex and nuanced process, which may be steered in different directions by a number of factors. 


\section{Methodology}

In this chapter, the methodology adopted for this study is described, as are the participants and the procedure of exploration. A study involving deaf participants rather than hearing ones poses a number of challenges, and differs from a hearing study in some ways. Firstly, Deaf people, who use Sign Language, might have different world-views and express those differently than hearing people. But even deaf persons whose dominant language is the spoken language of the majority, are at a disadvantage because of their hearing loss, and this colours the way they conceive of society and social interactions. That the researcher and the participants hold different world-views, then, indicates that the typical issues related to validity are present in research related to deafness.

Deafness does bring its own validity issues as well, which are principally related to language, but also related to the relationship between researcher and participant. These, and the above issues, are presented in section 4 of this chapter. Presently, I provide a description of the theory in which this study is rooted; this is followed by a brief account of the participants (they are discussed in more detail in the next chapter), and an explanation of how the data were collected and analyzed.

\subsection{Methodological Underpinnings}

The type of research I undertook was a cross between phenomenology and case study (Creswell, 1997): the focus was on understanding the intrinsic nature of identity construction among a few deaf individuals, and this was done by taking a close look at their perceived developments. Phenomenological research seeks to understand what constitutes experiencing an event or circumstance, and how events or circumstance relate to identity. Data is gathered by conducting interviews with participants who have lived 
the experience of interest, and analysis of the data produce themes that are assigned important meanings. Case studies are constructed by collecting data from multiple sources: participants may be interviewed, and surroundings and natural settings may be observed. Data produce themes that are used to build a detailed explanation of this case or these cases.

The goal of this project suits both phenomenology and case study: by collecting data from my participants, and examining the themes that emerged from their narratives, I intended to produce an explanation of how the social experiences of young deaf persons affect their identity constructs.

Some of the underlying, fundamental concerns of quantitative methodology such as accepting and seeking similarities across behavior are implicit, indeed are entailed by a concept such as social category. On the other hand, accepting that identity is emergent, negotiated, and dynamic as far as social interaction is concerned, leads the researcher toward a qualitative methodology in which the richness and variability of language in use is emphasized. (Pittam, 1999, pp. 115-16)

What Pittam (1999) is underlining is the importance of taking a more intuitive approach when examining something as fluid and personal as identity construction. For this to happen, a large sample is unnecessary, as a large sample may not be able to shed more light on the issues than a smaller sample. Indeed, my research was conducted with the help of a very small sample, which is described below. And this sample, as I show in the following sections, provided a variety of frames of reference with which to conceptualize identity among deaf persons.

\subsection{Participants}

For this project, six individuals with hearing loss were recruited. The size of the sample reflects my intention to look closely at those interviewees, and present their 
experiences in detail. Indeed, the size of the sample remains true to case study principles and avoids the necessary shift to cross-case study that a larger sample would require (Gerring, 2007). As well, a smaller sample was necessary for the time required to interpret and transcribe the interviews (more details about the transcription process are provided further down). For the scope of this project, six participants were a manageable group, yet one that provided the quality of responses necessary to present a worthy discussion of the subject matter.

All the participants involved, save one, were acquaintances or friends; a colleague referred the other participant. There were both benefits and drawbacks to interviewing people to whom I had relatively easy access. One such limitation was that the range of educational backgrounds was narrowed. The negative implications of interviewing participants with similar backgrounds means that the potential for diversified narratives is restricted, and comparisons become difficult. However, there is a positive aspect that needs pointing out: my participants are an approximately proportionate reflection of the wider population, in that their educational experiences vary in parallel ways to what Statistics Canada reports.

According to Statistics Canada's 2006 Participation and Activity Limitation Survey, $56.7 \%$ of children with disabilities, aged 5-14, receive regular education, while $43.1 \%$ receive special education (26.9\%: part-time special education; $16.2 \%$ : full-time special education) (table 2, page 9). Canadian children who were reported to have a hearing disability made up approximately $11 \%$ of this group (chart 3, page 11 ). As well, in the Participation and Activity Limitation Survey 2006, Facts on Hearing Limitations, by Statistics Canada, $15.3 \%$ of Canadians (aged 15 to 64 ) reported that they had attended 
special education school or classes (table 2, page 3). Statistics Canada does not define what is meant by regular versus special education, but the Ontario Ministry of Education provides special education in the form of itinerant teachers, separate classes, as well as provincial schools geared towards teaching specific groups of pupils.

What Statistics Canada's reports show is that deaf pupils are a small minority within the system of education and that they are very often placed in mainstream classes with little, if any, support for their needs. My sample approximates these statistics on a much smaller scale. Thus, although my sample is limited in terms of variety of backgrounds, it nonetheless is roughly representative of the greater population of deaf people in terms of educational experiences.

My participants ranged in age between approximately 20 and 35 years old. My sample was not limited when it comes to degrees of hearing loss and ages of onset - most of the participants deviated from the hearing majority in a significant way - and these details, as well as those relating to family and social backgrounds and school experiences, are expanded upon in the next chapter.

\subsection{Data Collection and Analysis}

The first step in the research process was to write interview questions. To be able to discover more about the phenomenon of being deaf, I required first-hand accounts of deaf persons' experiences. To get at these, I created open-ended interview questions to encourage the participants to share as much of their experiences as possible, and to encourage the development of a narrative. The questions were meant to lead to other context-dependent questions. See appendixes A and B for a list of the initial questions 
used during the interviews, and interview transcription samples which show some of the evolution of the questions.

Next, I interviewed the participants. The interviews were semi-structured, where I asked open-ended questions that were designed to lead to free-form narratives. All the participants were given a choice as to which language they wanted to conduct the interview in (either English, ASL or a mix of spoken and signed languages). All interviews were recorded and later transcribed. Interviews lasted anywhere from 30 minutes to an hour and a half, depending on the participant; the total hours of data amounted to about 335 minutes, or about 5.5 hours.

Once the interviews were transcribed, they were sent back to the participants, so they could verify that their intentions were properly represented. This participant check also allowed for some further inquiry when comments were unclear, or when comments were of particular interest. Finally, this part of the process also allowed participants to omit comments they felt did not do their narratives justice.

When all the data were collected, instead of imposing expectations on the participants' discourses and making their narratives fit into these molds, an effort was made to allow the themes to emerge on their own. Of course, some very broad themes had already been proposed and these were used as a starting point. Comments made by the participants that spoke to these topics were noted, but others that seemed not to match were also noted. Indeed, some comments related to more than one theme at a time and new themes also emerged. At times during the analysis, when a participant's narrative became ambiguous, I used my intuitions, as the researcher, to posit a particular underlying theme and I then examined the data for evidence elsewhere in their interviews 
to support, counter or elaborate on the theme. As a (hearing) member of the Deaf community, my knowledge of deaf persons' early experiences affords me an insider's understanding of the issues my participants recounted, and this understanding gives me "member's competence" (Woods, 1996). Of course, I was careful not to assume too much, and was able to limit my need to intuit by the process described above, whereby the participants were given the transcripts to review, with questions to clarify their intentions.

\subsection{Issues in Validity}

Research involving deaf persons presents some unique challenges. In this section, I describe three challenges that are directly related to having deaf participants and writing about deafness from a social angle. The first issue relates to when research involves friends or friendly acquaintances, and what that means for the interviews. The next issue is about language: interviewing deaf people poses language problems such as barriers or the use of non-native languages. Finally, terminology is also an issue because the language of deafness is Hearing-centric, and this causes problems when one approaches the topic from a hearing perspective.

\subsubsection{Research and Friendship}

As mentioned earlier, I had previously established a relationship with all but one of the participants, therefore, the dynamics of the interviews were further complicated. On one hand, a rapport of this sort may have helped to render the participants more comfortable in what would otherwise be a formal setting. They may have felt freer to divulge certain opinions or sentiments. Tanggaard (2009), however, holds that the interview is a unique setting whereupon participants are in fact constrained in the way 
they can respond to questions. There are, at times, certain power dynamics that determine the dialogical styles of narratives recounted by interviewees. My previous relationships with three of the participants were initially ones where I held a role of power: we had met in a classroom context, where I taught them, but had since then socialized in informal settings. So from my perspective, our relationships had shifted from teacher-student to ones of relative equality: friendship or acquaintance-ship. Whether this was true for my participants remains unknown.

Tillman-Healy (2003) argues that the process of qualitative inquiry is often one very closely related to friendship: “... friendship as method involves the practices, the pace, the contexts, and the ethics of friendship" (p. 278). The fact that I had known my participants on a personal level for some time (at least a year at the time of interviewing) had allowed me to set a foundation for the interview questions I asked of each person. Viewed through this lens, my previous relationships with my participants can be seen as beneficial to my research, since friends are generally more likely to be upfront with one another than with strangers. As Tillman-Healy explains, participants may feel more comfortable due to a sense of being understood and empathized with.

On the other hand, one difficulty a connection of this sort might cause is that the process of establishing a friendship tends to include the sharing of personal anecdotes. It then becomes a challenge to elicit bits of histories that had previously been divulged outside that setting, during an interview. In fact, the issue of identity had not previously been a topic of conversation between my participants and me. I thought that bringing it up in this context, then, would be fresh and would not feel as though we were having an artificial exchange. Despite the novelty of the topic, one participant answered the 
questions in a very concise way, but some of the others saw this as an opportunity to share the subtle nuances of their experiences. My awareness of this limitation did become a deterrent when I decided who of my acquaintances I would approach to take part in my research. It also led to my opting not to interview a close friend of mine specifically because I was concerned that I would not get the full extent of this person's history in an interview setting.

I had initially hoped to interview an equal amount, or majority, of Deaf school attendees; however, the data collected from the present group were potentially more interesting. My reading of the literature and especially my close friendships with deaf persons who had attended residential schools, meant that I was already familiar with the outcomes of deaf persons attending schools for the deaf; I was relatively unfamiliar with the consequences of deaf children attending school in the mainstream.

\subsubsection{Language and the Interview}

As I briefly explained in the introduction to this paper, I have been connected to ASL since the year 2005. In fact, my connection and involvement with the language and the Deaf community is quite deep. While I worked towards a minor in ASL, I also assisted my instructor. In addition to extensive classroom work, I often facilitated communication between my Deaf and hearing colleagues. My affinity for the language motivated me to become an active member of the Deaf community and I quickly made friends with some of its members. After completing my minor, I started to teach ASL at the university level. Since then, I have taught first and second year courses, and supervised teaching assistants in the program. 
Through my training, experience and socializing, I have acquired a thorough knowledge of the language. I have especially gained an intuition about ASL use and the interpretation of ASL concepts into English. Because the link between English words and ASL signs is not straightforward, a deep understanding of the semantic elements of signs is necessary to translate these into English words and ideas. My confidence in my signing abilities meant that I was able to offer my participants the option of having the interview conducted in ASL or English.

Before the interviews began, each participant was given this linguistic option. Although I was pleased to be able to conduct interviews in the language of each participant's choice, this entailed issues that I had not entirely anticipated. Three of my participants were native users of English, and they chose to have me use spoken English only, during the interviews. Of these three, however, one spoke a second-languagelearner style of English (implications are discussed in example 1, just below). Two participants chose to have the interviews take place in ASL, but only one of these two was a fluent signer. Finally, one of the participants opted to have me supplement my speech with signs. Below is a description of the difficulties I encountered with regards to conducting and transcribing certain interviews, using three different participants to illustrate these challenges.

I designed my interview questions so as to prompt spontaneous information sharing. I intended on giving all the participants the opportunity to interpret the questions in their own way; reflect on their experience; answer as much or as little as they felt comfortable with; and most of all, not influence their answers. This tactic didn't translate to Sign Language in quite the way I expected. 
My first interview was administered in ASL. This language has different pragmatic norms ${ }^{3}$ than English: directness is a typical characteristic of dialogue and so one of the participants who requested the interview be conducted in ASL answered my questions very briefly. My general questions - inadvertently designed for English speakers - elicited general answers. This entailed a difficult situation: because the answers I received were fairly superficial, there were few opportunities for me to have those answers lead to other, contextually relevant questions.

Example 1

Prior to the interview, this participant expressed a preference for English as the language of interaction for our purposes. She explained that she felt English to be a more specific language, whereas ASL was too general; she was concerned that her thoughts would not be properly portrayed by Sign Language. While English is her first language, it is not her family's first language (theirs is Polish) and so her English resembles that of a second-language learner's.

This participant was put into speech and listening training sessions from very early on, which she continued to attend until her early teens. The results of this intensive tuition are that this participant has fairly clear speech and a near-average ability to understand speech at a relatively close proximity; however, what is lacking is a thorough understanding of verb-tense agreement. During the transcription process, this matter became a challenge as I questioned my system of transcription. Was it better to be true to the participant's actual words or was it better to 'fix' those grammatical errors, for her

\footnotetext{
${ }^{3}$ As far as I know, this is not something that has been identified by researchers. It is an observation I have made whilst socializing with the Deaf community, and it is something Deaf people take pride in.
} 
benefit? I finally settled on transcribing as close to word-for-word as possible with the intention of editing whatever passages I later include in the report.

As I worked on this project, it became increasingly important to me not to change her words, however. The reason for this is that her grasp of English illustrates perfectly the difficulty that deaf persons have to learn spoken languages. Despite extensive training, when one cannot hear the input, it becomes a great challenge to properly reproduce the target language. And part of my goal in presenting this research is to shed light on the difficulties associated with trying to act as a hearing person when one is deaf. This participant's English proficiency is a symbol of the barriers that never disappear for those people.

\section{Example 2}

In the case of this participant, the issue of transcription was different than the one just mentioned. His interview was conducted in ASL and so the process of putting into writing what was signed to me was especially arduous, despite my fluency. This process involved further interpretation on my part, since the sense of a sign does not always correspond to an English word in a straightforward way; this forced me to consider very carefully what he meant to express. Also there exists no conventional transcription system for signed languages. As underlined by Senghas and Monaghan (2002), transcribing signed interviews is a methodological challenge caused by the lack of a rapid sign writing system.

Certain alternatives are possible to get around this issue: some researchers use illustrations to represent signs (particularly appropriate for the production of dictionaries; less so for documenting discourse); glossing - using a spoken-language word to represent 
the meaning of a sign - is much less time-consuming and is a fair, albeit crude, way of transcribing signs, and the product can look like a transliteration; elaborated glossing uses the same word representation method, but also includes supra-segmental and grammatical information, often represented on more than one line (see appendix $\mathrm{C}$ for an example). Quite a few more transcription methods have been proposed by a variety of researchers; for a more elaborate survey of the various methods of Sign Language transcription, see Senghas and Monaghan (2002).

Although this interview was the shortest of all, it took the longest to transcribe. For the initial part, the part with the most uncomplicated questions, I was able to watch the interview and type what I saw using elaborated glossing. But as the questions, and consequently the answers, became more complex, it became apparent that this process was not going to be time-efficient. I recruited the help of a copyist, to whom I recited the dialogue, as I saw it. This too, however, involved a learning curve. Because I am familiar with ASL and I can easily swing back and forth between it and English, the structural differences do not occur to me, let alone disorient me. As I vocalized the interview, my copyist often asked me to re-phrase my interpretation, or asked for clarification.

Despite this process being an improvement on my earlier attempts, it was nonetheless challenging and time-consuming. I used the copyist for approximately one third of the interview and finished the final section myself, by transcribing an ASL-toEnglish translation. The initial part of the transcription is a mix of ASL glossing and English, often looking like nonsense, especially to someone unfamiliar with ASL and its structure, while the final section looks like English. 
Four of the interviews were video recorded using a DVD-camera so that both auditory and visual data could be captured. For the other two interviews, only auditory data was recorded; the camera lenses were left on. The interviews were then transcribed into written English and these documents were sent to each participant for review. This review allowed the participants to examine what they had expressed and decide if in fact these responses represented their opinions and thoughts properly.

\section{Example 3}

A third participant chose to have the interview conducted in ASL. Of interest here, however, is the fact that she is not a fluent signer. This participant started to learn to sign approximately two years ago.

Our initial relationship was that of teacher-student, as I had recently taught her an intermediate level of ASL. Although she is a skilled signer for her level, she only has two years of experience with the language. This meant that the interview was conducted in a different way than the others: there were times during the interview when I had to return to my role as teacher.

On one occasion, she struggled to express an idea that the Deaf community finds very important, and that is for a person to 'think-hearing'. This expression means that a person does not understand the plights of Deaf people, that they value Hearing ideals and that they have no qualms about it. It is such an important concept to Deaf culture that there exists a sign for it. This sign is not a sign I typically teach my students, as it is so context- and culture-dependent; there is seldom an opportunity in class for me to teach it. But in that instance, I was inspired to share this cultural knowledge with her, despite the context of our meeting. 
There were also times throughout the interview when she used signs that she had picked up in social situations, which I was unfamiliar with (these were Englishinfluenced signs, which we do not condone where I teach). As well, some of the signs she used were mis-produced. In both these instances, I had to ask for language-based clarifications and I provided corrections where necessary.

\subsubsection{Terminology}

The limitations of the terminology that refers to different aspects of deafness in the majority hearing culture and in academic publications has been a significant issue in the writing up of this study and its results. These limitations develop because in the majority hearing culture, it is taken for granted that the language that defines deafness is founded on medical ideals. That is, generally speaking, deafness is conceptualized in terms of what is missing, and it is seen through a medical lens. Terms such as hearing loss and hearing impairment focus on how deafness deviates from what is 'normal'. In the literature, deafness is often described by words such as impediment, suffer, decline and affliction; again, these all denote negation.

In this thesis, I have attempted to avoid Hearing-centered descriptors of my participants' situations. In an effort to remain neutral and avoid judgments about my participants' bodies and lifestyles, I have used the term auditory context to refer to the situation, which I use throughout the next two sections. By auditory context, I am referring to the situation of being deaf, or differently-abled with regards to the auditory and visual senses. It is not meant to indicate, or speak to, the medical implications of deafness, but rather to signify the greater consequences of deafness, including the participants' environments and the atmosphere of their social conditions. 
My preference for auditory context does not change the necessity of referring to the medical condition of deafness in some instances. For this reason, I make use of the expression hearing loss when I intend to discuss the participants' hearing status in medical terms. Although loss implies a negative condition, it is the term that carries the least amount of judgment, in my opinion, and this is the reason for which I selected it. 


\section{$4 \quad$ Participant Profiles}

This chapter gives a detailed account of each of my participants, whose names have all been changed for the reporting of this study. In order to properly present what the participants revealed during their interviews, first, I introduce each participant in some detail, giving accounts of each of their backgrounds. This includes their ages, ages of onset, family backgrounds, schooling backgrounds as well as socializing tendencies (in that order). Their profiles also give a brief account of how the participants see themselves in relation to the world. Following each profile, I comment on what was said during the interviews as well as how those unfolded.

\subsection{Ben}

Auditory context: Ben is a male in his mid 30s who was born deaf; his hearing loss was discovered when he was two-years-old; his degree of deafness puts him in the profound range ( $\sim 90 \mathrm{~dB})$. His immediate family members are all hearing, but some of his distant cousins sustained hearing loss after birth. He made use of hearing devices when he was a child (an FM radio system), but stopped wearing any sort of hearing aids early on. Today, he does not use any hearing amplification devices at all.

School and accommodations: Ben was mainstreamed in the public school system, in the 1980's, until grade 6, where he was placed in a special class for students with a variety of learning disabilities, as well as deafness. In grade 6 , he was finally sent to a residential school for the Deaf. Once moved to the provincial school, he ceased to receive accommodations. After high school, Ben attended a hearing college, where he was given the use of two interpreters as well as note-takers. 
Language: In the public school system, teachers used Signing Exact English (S.E.E., a manually coded system of English) as the primary mode of communication and there was a heavy emphasis on learning to speak English. Through this training, he gained a reasonable understanding of English grammar. When he transferred to the school for the Deaf, he quickly picked up a more natural Sign Language (a pidginized form of ASL). At this school, the language of instruction was ASL, which he learned very quickly. He had some knowledge of signs, as given to him through his S.E.E. instruction, so picking up Sign Language was relatively easy. He learned Sign Language principally through the input provided by his peers, although all the teaching and support staff used ASL as well. All the students signed and so learning to sign was a requirement if he wanted to socialize with his peers. Although he went through speech training in English, today, he does not make use of his spoken language skills in a meaningful way. English plays a utilitarian role: he uses it when the situation requires it, such as when he is at work, with extended family, or with any hearing person who does not know Sign Language. Conversely, Sign Language plays a social role: he uses it with friends and in the world in which he feels he belongs.

Social life: Before Deaf school, he was relatively isolated, socializing in school, but seldom at home and on weekends. His life was mostly lived at school: weekends home were spent with his family who does not sign, and his summers were also socially barren. He was first exposed to the Deaf community during a visit to a school for the Deaf. Before that, he knew a few deaf children of deaf families, but had less contact with them than his other classmates, since those children signed better than he did. After his first visit at the Deaf school, he was persistent with his mother for her to send him there 
instead. When he finally arrived, he found it to be a new world and he made a concerted effort to become part of it.

Identity: In his earlier days, he was protective of his Deaf identity, choosing to associate almost only with other Deaf people. Now, he is less focused on the hearing status of people he chooses to surround himself with; he bases decisions related to friendship on the attitudes of people. However, he does not seek out friendships with hearing people unless they enter his world (many of his hearing friends are ASL students).

Ben sees himself as a functioning member of society, capable of doing a normal range of activities, albeit with the help of accommodations at times. He does not equate deafness with disability: 'I don't label myself as disabled, I'm deaf, that's it. Most people look at us and think we are, but I can't hear - that's all.' He belongs to the Deaf world; the hearing world involves pragmatic norms that he is not accustomed to and has difficulties negotiating. However, he is aware of the value of being comfortable in the hearing world because he recognizes that it is an unavoidable part of life.

Comments on the interview

This interview was the most disappointing one for a few very important reasons. I came into this interview with big expectations: I knew that this participant was the most involved in the Deaf community and that he had gone to a Deaf school, therefore his perspective would be distinct from the others'. Of all the participants, he and I also have the closest friendship, and I know things about his background that I thought would add a lot to my research. What happened during the interview, however, was anticlimactic. Ben chose to have the interview conducted in ASL, which meant that I had to reformulate my 
questions as we went along. My questions were formed in such a way that there was room for interpretation on the part of the interviewee - I did not want to encourage my participants to answer one way or another - but Ben answered these questions casually. His answers were superficial, to the point and general.

While this represents pragmatic aspects of Sign Language ${ }^{4}$, it did nothing to add to the complexity of his situation. In fact, it removed all the colour from his stories, making them plain and sober. Because of our friendship, I know this person has a more complicated background; I cannot help but wonder why he chose not to divulge that when he was prompted.

I believe that what took place was a product of our relationship. First, there is a conflict felt by the researcher in this situation: which "hat" does the researcher put on in that setting - friend or authority? Because we were already friends, my need to act as an interviewer changed the dynamics of our interaction and perhaps my choice was restrictive and led to the superficiality of the answers I was given. Next, this particular participant had already shared much of his life stories and I had seen him discuss issues related to my topic in social situations, which meant I had a substantial amount of previous knowledge. Because I wanted him to express himself freely, I did not assume anything while I asked the questions. Instead, I accepted the answers as he provided them and only prompted him to further explain in a general way, without steering his answers. At the end of the interview, he told me he felt I had not really asked anything of substance and said that if I wanted to ask him any further questions, that I should go right ahead.

\footnotetext{
${ }^{4}$ Users of ASL seem not to add extra information when asked a yes/no-question. They only elaborate on wh-questions.
} 
It is difficult to interview a friend in this context, then, because they expect you to use the knowledge you already have either to formulate your questions, or to guide your reflections. Unfortunately, ethically, a researcher can do no such thing without the participant's consent, which is most clearly given by the participant restating those anecdotes that have already been told.

\subsection{Jessie}

Auditory context: Jessie is a female in her mid-20s; her degree of deafness puts her in the profound range $(\sim 95 \mathrm{~dB})$. Her family is made up entirely of hearing people, but she became deaf in early infancy, apparently due to an irradiated vaccine. Born in Poland, her family moved here when she was an infant to have access to better hearing technologies. She wears one cochlear implant (CI), which she received when she was four-years-old. This is the only hearing device she makes use of.

School and accommodations: She spent all of her schooling years in the mainstream system where she had frequent contact with an educational assistant and isolated tutorial sessions replacing class time. After high school, Jessie attended a hearing university, where she received minimal accommodations (note-takers, primarily).

Language: After implantation, she started to receive speech and hearing therapy in English, which became her first language. Speech training continued through all of elementary school and some of high school. In elementary school, she was placed, unsuccessfully, in French lessons, but soon stopped learning this language to focus further on English. Her parents never taught her the family's native language, Polish, and she attempted to learn it recently, by taking a class. She uses English in most contexts of her life, such as at work and with her family, as well as socially. She started learning ASL 
a few years ago, after being prompted by a friend, and completed 2 levels. She now uses ASL in her social life, regularly. Her partner is Deaf, and he communicates almost entirely in ASL. She also uses Sign Language with other Deaf peers, whom she has come to know through her partner, primarily.

Social life: Friends play an important role in her life; she equates friendship with cooperation and help, often expressing appreciation for those friends who provided her with support in school or who now help her improve her hearing/speaking skills. Her early high school experience was difficult and marked by social exclusion and struggle. After her trip to Poland, where her cousins taught her how to joke, these difficulties ceased. She now maintains friendships with members of both the Deaf and hearing community. At the time of the interview, she felt that she was not yet an established member of the Deaf community ("part-time in the Deaf community"), and didn't seem to feel that she had any agency in possible developments ("Some Deaf people, they don't... contact me much or say, let's go out, so... if that's what they want, I don't [mind]"). In fact, at that time, she felt that she was more in the hearing world because of affiliations through school, work and friends.

Identity: She belongs more to the hearing world but is part of other communities as well. Despite her Cl, she does see herself as deaf, but this isn't her most defining feature: "I do see myself as a deaf person... with cochlear implant... But if I take it out and show my real self, a real human, then I'm deaf".

She reported identifying strongly with her Polish roots, and a trip to Poland during the summer after grade 10 awakened a stronger sense of self in her. This newfound identity was so meaningful that it was manifested by a change in her physical appearance; 
she changed her hair and the style of her clothes. Ironically, though she values her Polish identity, she also associates it with traditional values and closed-mindedness. Since she sees herself as being open-minded, she reconciles this contrariety by describing herself as a rebel, and explains that she is sometimes an outsider since her views conflict with her family's views.

She is comfortable in her own skin, confident and proud of her accomplishments. She sees life as a series of difficult tasks and obstacles that require dedication and hard work to overcome, and she gladly accepts those challenges. She views learning as a shared and negotiated process; she is open to trying new things and listening to others' perspectives to gain an understanding of their perceptions.

She believes her most important traits, those that represent her best, are her outgoing-ness and her focus on having fun and creating fun environments for her friends. She talks about jokes, pranks and teasing very often throughout her interview. Humour is obviously a very important socializing medium for her.

Comments on the interview

In this interview I could clearly see the participant normalizing her experiences, her situation, and her own reactions too. When she recounted her experiences with kids teasing her, she explained that this is what children do, that her reactions were standard and that this sort of interaction was normal: "[In] kindergarten... I was being excluded... I tried to make friends, it was hard for me to make friends because they sometimes joked to me, or something like that, and I don't know the jokes, I don't know the songs and I'd get frustrated... I start to yell and say a few sentences and then a little bit of gibberish... It was kind of frustrating because friends, they know how to pick on and I don't know 
how to deal with it at that time... And afterwards, it's like, you get angry one day and the next day, you're friends. That's the thing, you know, with children".

She attributed a lot of her behaviours and experiences to being a "normal kid":

"When I have a question - I don't really ask my teacher for questions, when I come after the class... you know, I was a kid". But nothing about her experience is normal. She came to Canada when she was a child, so her parents were not fluent English-speakers. Her childhood might have been very lonely due to lacking means of communicating with her parents, and a shortage of friends. Only when she was able to connect her identity to her parents' was she able to find her place in social circles at school.

Since this interview took place, Jessie has moved in with her boyfriend and has become an active member of the Deaf community. She organizes social gatherings for large groups of Deaf people and her circle of friends now includes many more Deaf people than when I first spoke with her. I suspect that many of her answers have now changed, however, she declined to be interviewed again.

\subsection{Amy}

Auditory context: Amy is a hard-of-hearing female in her early-mid 20 s. Following a series of ear infections, she sustained unilateral loss of hearing, which was diagnosed in her late childhood. Her family members are all hearing ("normal", as she puts it). She was fitted with a hearing aid in grade 8 and has since worn a hearing aid in one ear.

School and accommodations: All of her schooling was done in the mainstream. She had a mostly 'normal' experience in school, learning to play an instrument and never attending any special classes. The extent of the accommodations provided was extra time 
on tests and special headphones for second language tests. After high school, she continued on in a hearing university, receiving the help of a note-taker.

Language: Amy learned English as her first language with little difficulty and she uses English in all aspects of her life. She started to learn ASL a few years ago and completed 3 years of study. She has not, however, incorporated it into her life in a meaningful way. When asked why she initially wanted to learn Sign Language, she said: "It'd be kind of fun [...] to learn a language where I don't have to worry about listening". Social life: Her friends are mostly hearing, but she has at least one hard-ofhearing friend. She communicates with all her friends through speech alone (Interviewer: "How do you communicate with your hard-of-hearing friend?" Amy: "Well... obviously, speaking"). She is squarely rooted in the hearing world, seeing hearing as normal: interviewer, "is anyone else in your family deaf or hard-of-hearing?" Amy: "None; parents are fine, brother's perfectly fine". At the peak of her involvement with ASL, she signed with classmates during ASL class, and sometimes outside the classroom with those same peers and a few Deaf peers. Amy no longer attends ASL class and has since stopped socializing with ASL users. Her social life now consists primarily of interactions in the hearing world.

Identity: Prior to being fitted with a hearing aid, she did not consider herself hearing impaired: "I didn't identify as having a real hearing problem until I had that hearing aid". She received the hearing aid in late elementary school and wore it until high school, when she stopped wearing it. She explains that the stigma attached to wearing a hearing aid is what prompted her to leave it off. She also describes feeling as though her peers saw her as having a weakness when she wore the hearing aid and that she also felt 
this about herself. Being fitted with a hearing aid was a personal defeat since she had "made it that far without one".

Comments on the interview

The personal evolution Amy presented in her interview was characterized by her acceptance of technology and by her intent on making her hearing context visible. When she walked into my office for the interview, Amy opened our conversation by drawing attention to her new hearing aid. She was excited to show me how advanced it was; loaded with Bluetooth technology, this device can wirelessly play music and act as a phone receiver. She was obviously proud of it.

The interview was structured chronologically though, and so initially, she talked about her hearing aid causing her social harm and altering her self-image. Before being diagnosed as hard-of-hearing, Amy was a quirky, musical student. She had her "own" language (arguably a speech impediment due to her inability to hear clearly) and played her instrument very well. After her diagnosis, she became "disabled", struggled in school and did not fit in with peers anymore. She recognized that there was a stigma attached to wearing a hearing aid and that it changed people's perceptions of her. This led her to avoid wearing it while she was in high school.

But when she shared her current feelings about her context, it was clear she preferred her deafness to be visible. For Amy, her deafness is about missing, lacking and less, as evidenced by the language she used throughout her interview. To cope, she had turned her deficiency into an advantage, or a hardship that had advantages. Her response to my asking whether her ear operations resulted in scarring was, "I wish!". She also talked about turning her operations into something cool rather than lamentable: laughing, 
she explained, "the last one was actually cool, I had staples in my ear [...] I went to work after I was recovered, with the staples [...] and I work in security, so we had metal detectors, so some of the guys thought it was funny and said let's see if it goes off'" As a visual (and visible) language, it is not surprising Amy was interested in learning ASL, since it, too, could enhance the visibility of her situation. By making her deafness visible and interesting, Amy is challenging the stigmas attached to it.

\subsection{Steph}

Auditory context: Steph is a hard-of-hearing female in her early 20 s; her degree of deafness puts her in the moderately-severe to severe range $(\sim 70 \mathrm{~dB})$. Her hearing loss was diagnosed when she was in grade 3 . Apart from a few remote family members with varying degrees of hearing loss, she is the only member of her immediate family with a hearing impairment. Steph wears hearing aids in both ears.

School and accommodations: She attended a mainstream school for all of her schooling and received some remedial help while there. After high school, Steph entered a hearing university and has been there since. She receives the help of note-takers, who allow her to take short breaks during lectures, as she notes that trying to listen to lectures is very tiring.

Language: English is her native language. She started to learn ASL a few years ago, and has since become somewhat involved in Deaf activities. Though English remains her dominant language, she now has a few Deaf friends, with whom she signs, and some hearing friends with whom she can use ASL as well. Of learning to sign, she says: "I do [see it as something that would help me] now, I don't know if I did [at first]. 
[...] I just always thought it was cool and fun". Still, she uses English in all other aspects of her life.

Social life: Steph was bullied and excluded on a few different occasions throughout school. She changed schools in grade 1 for this reason, and peers were "nasty" again in her new school. Socially, she struggled for good part of her school years. Things seem to have improved in high school and she finally found a community she feels akin to while at university. Today, her social life is full. One of her strongest affiliations is to the engineering community (within the hearing world). The majority of her friends are hearing, but she does socialize with members of the Deaf community.

Identity: Steph identifies as hard-of-hearing and distinguishes that from deafness. She feels connected to both the hearing and the deaf world, to differing degrees: she has experienced similar struggles to those of deaf people and has trouble with 'hearing' technology (telephones, for example). Still, she doesn't identify as "deaf-deaf", something she associates with poor performance at school: “obviously I can't relate to being deaf-deaf... I've always done well in school and whatnot".

Meeting Deaf people has helped her to understand herself a bit better though. She says she always felt different, but didn't know why and accepted labels from others. But after meeting another hard-of-hearing student who made her feel normal for her lack of focus and falling asleep in class, she better understood herself.

Comments on the interview

This interview was really interesting because it read like a personal evolution in two areas. At the beginning, Steph displayed a really acquiescent side of herself. In fact, this passive acceptance of other people's decisions, desires or judgments was a recurring 
theme in her interview until about half-way through. Before her hearing loss was diagnosed, her teachers labeled her a daydreamer and troublemaker. Although she wasn't convinced the label fit, she went along with it. When I inquired as to whether this seemed ill fitting to her - a misrepresentation of her intentions - Steph seemed unfazed: "I was a little kid - I didn't care about anything". To a certain extent, she seems to associate being young with living experiences that are inconsequential in the long term. Interestingly, for her, this seems to have been true. After she started university and joined the engineering community, it seems she became more assertive or more interested in forming her own opinions of herself.

The other area in which Steph seemed to evolve was in her opinion of deafness. Throughout the interview, Steph swung back and forth between two extremes, sometimes indicating that she saw deafness as unremarkable, and sometimes treating deafness as a condition that sets people up to be very different. By the end of the interview, although she chose not to declare membership with the Deaf community, she nonetheless took ownership of Sign Language; or rather she dissociated herself from spoken language. She claimed that "my" language (spoken language) was less efficient than Sign Language. It seems that she "gave" me spoken language because of my hearing status.

\subsection{Joey}

Auditory context: Joey is a male in his mid-20s who lost hearing in the first week of his life; his degree of deafness puts him in the moderately-severe range $(\sim 60 \mathrm{~dB})$. His whole family is hearing. Joey was diagnosed the earliest of all the participants, when he was a few weeks old, through infant screening tests. 
He has worn hearing aids since he was one, and received a cochlear implant in his early 20 s, which is unusual for two reasons. First, the medical community prefers to implant people when they are younger; second, because his degree of hearing loss isn't what would normally warrant a CI. He was implanted as part of a study, and his acquisition of the implant was experimental. He now wears the $\mathrm{Cl}$ in one ear and a hearing aid in the other ear. He is very happy with his hearing devices.

Schooling and accommodations: He was put into mainstream schooling from the beginning and made use of an FM system in most classes. Because he was bullied in high school, he transferred to an alternative school in grade 11. After graduation, he took a few years off before starting university. He does not make use of accommodations to which he would normally be entitled.

Language: Joey was put into auditory-verbal therapy when he was an infant and continued this therapy for many years. His mother tongue is English and he does not speak any other languages, nor has he learned ASL. Of ASL, he says: "I don't really interact with signing people that much, so I don't necessarily think that learning signing would give me a whole lot of opportunities; I'd prefer to learn more French or even something like Japanese, before I'd sign".

Social life: In elementary school, he was not very social, with only a few friends. High school was very lonely as well, as was the beginning of university: "In high school [...] I went weeks without opening my mouth in school". Like some of the other participants, he was bullied in high school, but he does not link the bullying to his hearing impairment. He only recently started socializing and he attributes this to his implant. Joey 
explains that before the implant, following conversations was tiring and a struggle; since getting the implant, it's been easier to listen.

Identity: He sees his hearing loss as a disability, though he doesn't feel as though he is severely disabled. He thinks that, those areas of life in which he is handicapped, he can overcome with hard work: "it's that much harder for me to make the same progress that it would take a non-deaf person to".

He has no affiliation with the Deaf community and does not identify with them (he referred to Deaf persons as "signing people" more than once). Being an avid reader and writer, he feels that he would not be able to relate to their experiences ("especially knowing the literacy levels are very different in the Deaf community [...] it's just worlds different from my personal experience, so I don't really feel a connection..."). He sees Sign Language as a useful tool, but isn't especially eager to learn it: "there's a convenience aspect to it [...] if I'm just sitting at dinner and have a mouth full of hot food... being able to sign 'oh I'd like some water now please"'.

\section{Comments on the interview}

The most striking aspect of this interview was the sense of pride that came through when Joey talked about his involvement in the medicalization of the treatment of deafness. He made a point of mentioning that he was one of the first to be assessed under the infancy screening tests; that his auditory-verbal therapist was a pioneer in the field; and that he was implanted as part of a study since he wasn't a clear-cut candidate for the surgery.

Joey also mentioned that there was a discrepancy between his capacity for verbal output and his ability to hear, which led to difficulties in school: "there was a gap 
between how badly I heard and how well I spoke, so people misinterpreted I think, a little bit...". Even the use of 'badly' to describe his hearing is noteworthy: Deaf people don't use terms like this to describe their situations, but this is a learned behaviour. Deaf people learn that their hearing contexts are not a negative thing by taking part in Deaf culture.

This is related to the second striking aspect of this interview: his deeply negative view of deafness and deaf people and their language. During the interview, he spoke of some negative encounters he had had with deaf people on two separate occasions and made sure to distinguish himself from "signing people" (his words) by drawing on literacy disparities. He also gave ASL a purely functional role in life, explaining that it is useful only when spoken language is not an option. Even those situations, he trivialized to an extent: he suggested that ASL would be good when you need to communicate while your mouth is full, or that it is used in auditory-verbal therapy with patients who are severely disabled and cannot speak. As he explained, "my mom doesn't typically sign with her students $[\ldots]$ It's usually a result of a child having other, more severe disabilities where signing to supplement speech is necessary".

\subsection{Shayna}

Auditory context: Shayna is a hard-of-hearing female in her mid-20s her degree of deafness puts her in the moderately-severe range $(\sim 60 \mathrm{~dB})$. Her family is made up of hearing and deaf people. On her mother's side, all members are hearing; on her father's side, her father, uncle and grandfather are all hard-of-hearing or deaf. Her mother noticed she had problems hearing when Shayna was five-years-old, and her hearing loss was soon diagnosed. Shayna received one, and later, two hearing aids. She was fitted with her first hearing aid in grade two. 
School and accommodations: She spent all of her schooling in the mainstream system and was placed in a remedial class with an itinerant teacher early on. She was also tutored in the evenings. In high school she tried to pretend she did not have a hearing issue, but one teacher approached her and offered help. From then on, she received lecture notes ahead of time. After high school, she continued her schooling in college, where she received similar accommodations. Finally, she entered university and, again, receives the same sort of accommodations.

Language: Shayna's receipt of a hearing aid came with speech therapy. She had been exposed to English as her mother tongue since infancy, but had struggled to learn certain sounds. She also struggled to learn French, failing French Immersion classes in grade 1. She was tutored in French after that, and in college, after going to French class five days a week, for two years, her teacher told her she would do best with private instruction. During college, she started to seek out ASL courses actively and was finally able to get into a class when she arrived at university. She uses ASL regularly and claims that it will become her primary language later, when she loses all her remaining hearing: "Sign Language, it's like a plan for the future. Before, my hearing loss scared me; now, it doesn't. Sign Language, I think, is associated with that". Still, she wears her hearing aids in order to use spoken language to communicate with the hearing world.

Social life: Throughout grade school, she felt isolated and bullied. She stated that she did not have many friends until she got to high school. Through elementary school and some of high school, she tried to cover up the fact that her hearing was different than that of her peers, "Before, when I was little, I was nervous, I was still pretending I wasn't different, that I was the same as everyone". She became more social in high school and 
after starting to learn to sign, she started making Deaf friends. She now socializes with both hearing and Deaf people.

Identity: She is medically hard-of-hearing, but identifies more and more with the Deaf community, "[M]y hearing friends don't know Sign Language, so that's hard. Now I feel closer to my deaf friends, really much closer." She is now very involved in Deaf culture, socializing with other Deaf people and organizing activities. Since she has started learning the language, she has influenced her father, uncle and grandfather and convinced them to take it up too.

Shayna's relationships with her hearing mother and hearing half-brother are strained and unequal; both often feel that her hearing is selective and that she just lacks motivation and effort when it comes to communication. When she tries to speak to her mother, the latter often puts more emphasis on the delivery of a message than on its content. Shayna's mother also does not support her daughter's interest in Sign Language, believing that this interest marks her daughter's rejection of the hearing world. Her mother, therefore, does not sign.

With her father, uncle and grandfather, she feels more accepted and in turn, she feels closer to them. Shayna communicates with her male relatives by speech, writing and some (very limited) signing. Because all of their auditory contexts overlap, they do not judge each other and they do not "give up" on attempts at conversation.

Comments on the interview

Shayna's interview read like a clear evolution of the self. Although her transformation was not yet complete at the time of the interview, she did indicate in her interview that she wanted to cross over into the Deaf world. In her early years, she was 
confined to a world where she was made to abide by rules and conventions that did not suit her. In her more recent years, as she made a concerted effort to become a Deaf person, she learned to communicate more freely and with like-minded people. She reported feeling more accepted, now that she could claim membership to a group that felt like home. "Now I have more D/deaf friends and communication is easier".

Her transformation is held back in part by her fixation on the technicalities of her affliction. At the beginning of the interviews, I asked each participant about their hearing status. I hoped to get approximate numbers describing the decibel capabilities of each ear, and most participants were able to provide that (though not everyone could). Shayna, however, was not only prepared to give me that information, she had come with two of her audiograms: both her most recent one and her first one, from approximately 20 years ago. These allowed her to show me her exact hearing abilities, and to compare the two tests, which she offered to show me so I could better understand the progression of her hearing loss. Members of the Deaf community are not concerned with medical technicalities 5

This preoccupation with the medical aspects of her condition does not align with her extensive talk about joining the Deaf world. It speaks more to a reliance on the medical community's opinion of deafness, and she seemed to put a lot of importance on what her audiologist counseled. This also meant that she put importance on the hearing world's perspective of deafness, since the medical community and the hearing world tend to be one. While it is true that there are different ways to be Deaf, by contrasting Ben's and Shayna's interviews, it became clear that she was not yet prepared to commit to

${ }^{5}$ Ben did not know what his exact decibel levels were for each ear, and he reported not having had an audiogram in years. 
labeling herself as Deaf. Interestingly, her familial context might lead one to think that she would be more prepared than Ben, and yet, she was quite concerned with hearing opinions. While Ben's family is unsupportive of ASL in that they have not taken the time to learn it, he did not report there being any conflict at home. In Shayna's family, however, one side was extremely supportive of Deafness while the other was strongly opposed to that idea. This polarity at home seemed to have a salient effect on her sense of self.

One thing that came up often in this interview was the participant's opinion that deafness and socializing, and coping with deafness, were challenging things. She referred to many situations as "hard", from communicating with her mother and mainstream schooling, to getting used to hearing aids and grappling with friendships with nonsigners. She also talked about entering the Deaf community as hard:

I feel that, with the Deaf group, it's so much easier. There's also problems sometimes, though. Other Deaf boys, men, whatever, they become attracted very quickly. Like, last Friday, I was at [...] a social, one person who was there was [attracted to] me. [...] I tried to tell him to back off, to relax, but he wouldn't stop [flirting]. It's like, I'm deaf so I'm available. Like I'm another option for them. [...] So it's weird, it's hard.

There are clearly certain aspects within each community that make social experiences difficult, and this could account for Shayna, and most of the other participants, hesitating to commit to one side completely at this point. The next chapter offers a closer look at this issue, as well as issues relating to social interactions and relationships; the effects these have on identity and group membership; and finally, at the use of amplification devices and the results of these uses. 


\section{$5 \quad$ Results and Discussion}

While reviewing my data, I often wondered why someone who has difficulty hearing and who has been exposed to Sign Language would still prefer to live their social life in the hearing world. Why would they remain in this sphere where struggles abound and their differences set them apart in often-negative ways? After all, these people are perfectly suited for the Deaf community: they share formative experiences with other deaf people (regardless of efforts put into integration) and are seemingly ideal candidates for membership.

In an attempt to understand this situation, I reflected on my own experience as a novice runner. I started running two years ago. In an effort to restart my life after a significant separation, I quit smoking and took up jogging. My first few runs were difficult, awkward and, although I felt proud of myself at the end of a run, the process of going for a jog was trying. After a few weeks, I decided that I needed new shoes, so I headed to a running store. The sales person dictated all aspects of this experience; informing me of the type of shoe I needed, how it should fit, what size to buy -- not my own -- and so on. With my new shoes, I jumped into running more seriously than before; I wanted to call myself a runner.

I soon registered for a race, imposing a tangible goal on myself. I ran more often, bought more outfits, new shoes and this time, having experienced my body while running, I was able to dictate the way in which the transaction played out. Finally, race day came. I was nervous but exhilarated and I ran a race I was very proud of. This solidified my identity as a runner. 
More recently, I signed up for a running clinic where I would join a group of runners for training sessions. Surprisingly, I found that I did not fit in with this group, and I finally dropped out of the clinic.

My experience with running parallels that of my participants'; my process consisted of identifying a group I wanted to join; living some awkward moments during which I learned the language and behaviours of my target group; and finally, performing my newly acquired skill. My journey also included some socializing with members of the group, with whom I found that I had little in common- only our interest in running linked us, which was not enough to sustain a relationship.

Joining this group with whom I should have a lot in common does not seem like an important part of my transformation anymore. By adopting the behaviours of my target identity, and by absorbing them gradually, I was able to become a runner. So, although being a runner is an important part of my identity, the community of runners is not one that I am intent on joining. I am a runner, but I am most comfortable living that aspect of myself, by myself.

There are three aspects of importance in my analogy. The first has to do with the technicality of being a runner. By performing the activity, I run. Next, by physically running in the technical sense, I can then identify as a runner in the social sense: I am a runner. Finally, identifying as a runner allows me to choose to join the running community and become a member of the culture of Runners. In this sense, I might become a Runner. What I concluded above, however, is that identifying as a runner and joining the Running community are not inherently related, and one does not always lead to the other. Of course, continuing to run might lead to joining other runners and I might 
slowly become a Runner. This transformation would involve adopting the values and beliefs of that group.

As an analogy for what my participants may be going through, the levels are not quite the same. The first level parallels the medical condition that is deafness. The difference is that I have a choice in whether I run or not, and there is no stigma or discrimination associated with that choice, which means that these two technicalities (running and being deaf) do not exist in the same way. On the other hand, a deaf person may attempt to hear (by using technology), which corresponds to my analogy in an opposite way. Next, my participants may choose to identify as deaf in the technical sense, or as hearing impaired. Each term implies different value judgments: 'deaf' implies an acceptance of the auditory context, while 'hearing impaired' means that someone favours Hearing values and that they actively work to become more skilled at hearing activities (listening, speaking). It also implies that they are dissatisfied with their auditory context. Finally, someone who identifies as deaf might seek out the Deaf community and choose to join it.

In this chapter, I discuss the results of my research with regard to these three issues. In the first section, I focus on the early relationships of the participants and how communication took place during this time. These experiences are relevant to their identity formation, in particular their formation of relationships. I also discuss the technicality of deafness and how deafness affects relationships, both parental and platonic. In the second section I discuss their more recent perceptions of identity and deafness; I examine the issues of Deaf and $\mathrm{HoH}$ identity and group membership, particularly how my participants see themselves fitting into each category. In the third 
section, I focus on the ways my participants chose to influence or change their auditory contexts, be it through language and communication, or through technology. In this last section, I also offer a look at how decisions about identity affect attempts at group membership, in practice.

\subsection{Early Relationships and Communication}

The outcome of deaf children is dependent on communication (Yoshinaga-Itano, 2011). When there is a communication barrier, as deafness entails, this can pose a challenge for the formation of relationships, within and outside the family. Jessie, for example, started to learn to speak English - her only language at the time - when she was four; her parents, who had recently immigrated on account of her deafness, were just learning to speak English as well. Jessie's older brother was quicker to pick up English and he acted as an intermediary with doctors, and I assume he provided the same bridging at home as well, as she seemed to feel especially close to him, mentioning him much more than her parents in her interview. This linguistic atmosphere may have been impeded the formation of relationships between Jessie and her parents.

Even when the communication barrier is less present, there may still be troubles with communication that relate to deafness. Both Shayna and Steph reported some difficulty at home when it came to communicating with family members. Of speaking with her mother and sister Steph said, "they're stubborn, like, I'll be like, I can't hear that, and they'll be like, just try harder..."

Shayna described her connection to her mother as "difficult". She explained that communication with her mother was strained because her mother tended to give up on conversations if Shayna struggled to understand what was being said: 
My mom and brother, they give up. If the message is very specific, concise, important, then maybe say, there's a meeting or a doctor's appointment she has, then she explains... [But if] I'm explaining something to her, she'll tell me my voice is not good enough. She'll say, do it again, do it again.

When the types of communication are compromised like this - when the message takes a backseat to the quality of delivery - sharing becomes impossible. In a situation like this, a relationship may not have the same essence as one where communication flows freely, and this might impede social tendencies, as Bowlby (in Weiss, 1998) implies when he claims that "an individual (of any age) [who] is feeling secure $[\ldots]$ is likely to explore away from his attachment figure" (1998, p. 121, as quote in Weiss, 1998, p. 677).

Deafness in children may hinder communication between them and their hearing parents, and it may also hinder communications with their peers, in social and educational settings. School is, in part, meant to prepare younger generations for future interactions; it should train them to take part in sharing, communicating, and cooperating. School is often where a child's first true friendships are formed, and social acceptance at school is an important factor, but may be tricky for deaf children. Ben and Amy had the most socially positive school experiences of all my participants, despite the fact these two are at opposite ends of the spectrum, in terms of degrees of deafness (Ben being the most deaf and Amy being the least).

Given that the deafness of a child may disrupt communicative processes with hearing parents, it is plausible that the relationships formed at a residential school for the Deaf, between the councilors and the children may replace those bonds that would normally be formed between a parent and a child at home. It may be that the act of attending a segregated school, where children and adults with hearing impairments are grouped, leads to stronger relationships forming in this environment instead of at home. 
For many deaf children of hearing parents who enter a school for the deaf, this is their first experience with Sign Language and their first experience with adults and children with whom they can communicate easily and therefore form meaningful relationships. Due to the restrictions in communication deaf children may experience with their parents, those children may form different relationships than hearing children, focusing more intently on friendships. When deaf children are placed in these schools, they tend to live on campus for the week, and see their parents on weekends only. Ben, who attended a school for the Deaf from grade 6 onward, displays a need to form connections when he talks about arriving at the school:

[Sign Language] was everywhere. It was a new world. The signing was different and I picked it up really quickly. I met two or three other students in my class who were from Deaf families. Their parents and the generations before were all deaf. They were really good signers. So I was challenged by their skill to pick it up quickly. I tried to get up to their level quickly.

When a deaf child of hearing parents is placed in an environment with other similar children and adults, the child is likely to take advantage of this unique communicative situation. For Ben, school was a social hub. Even before being sent to a residential school for the deaf, when he was initially placed in a class for children with different needs, he had access to some peers with similar communication abilities and auditory contexts. "At recess we signed, but in school we couldn't" he explained. He also talked about socializing being limited to weekdays, as weekends were reserved for family time, where communication was limited. Ben explained that communicating with family was done "mostly through writing. Sometimes my brother interprets, but [it's] mostly written. If the message is short, he'll relay it, but otherwise, we write back and forth". 
Written messages do not convey the same emotions natural language does, and this could impede relationships.

When Ben arrived at the residential school, he formed strong bonds with his peers, which lasted a long time. Although his socializing experiences growing up were apparently positive, there was little emphasis put on socializing with hearing kids. These interactions he described as difficult: "If they knew a little bit of signs, we could negotiate, communicate, but if I had to teach them the signs, ugh, no. I'd show them a few things and then later they would forget". Today, all of Ben's meaningful associations with members of the hearing community are on his terms, meaning those hearing people with whom he enters into relationships must accommodate him by moving closer to the Deaf community. He claimed that he does not seek out relationships with hearing people, unless they are signers. He explained that this is an improvement for him:

Now I'm more flexible; when I was younger, I wasn't. I was protective about keeping a closed group of Deaf friends. Then... I went to college... [and] there weren't any Deaf people. I made a few friends there... I was part of a group... That was a good experience for me: growing up, I'd been able to stay nestled in my little Deaf group but in later life, that has to break.

The experience Ben described mirrors what Bowlby (in Weiss, 1998) explains above: that an individual must feel secure in an attachment in order to move beyond it. It seems, however, that the attachment he was able to explore away from, was the one he had formed with his schoolmates and possibly the teachers and staff at the residential school. Indeed, it is possible that the presence of his parents when he was home was not enough for him to form attachment bonds with and he, instead, was able to form these bonds with others at school, through visual communication. That Ben was once hesitant to socialize with hearing people, then, is not surprising. 
In my experience, members of the Deaf community have told me that they see the community as their family. What seems to happen, then, is that the residential schools for the deaf act as a village, and within it, deaf children form attachment bonds that can completely sustain them socially. If this is the case, it is possible that rather than children maturing into secure, curious individuals, the attachment bonds are nurtured and deepened within the circle (which includes both adults and other children), replacing the need for these individuals to seek attachments externally. The secure bonds formed in this community make it all the more difficult for these children to overcome the obstacles present in the hearing world.

Degree of deafness plays an important role in the issue of family relationships and social relations at school. Amy, whose deafness is the least significant of the group's, indicated that she always felt accepted by her hearing peers in school. When asked whether she felt that she was treated differently by her peers, she responded, "As a collective group, I would say no... I remember when I first got [my hearing aid] in grade eight, I told my best friend, 'I have a hearing aid'. She [was] like, 'that's cool'". Amy's deafness was surrounded by hospital visits and surgeries, but she claimed she was able to maintain a healthy circle of friends and partake in typical activities with the other children. The stigma that is normally associated with physical difference, as explained by Jones et al. (1984), seems not to have been a factor for Amy.

Deaf children may experience difficulties socializing, in particular when degrees of deafness are significant enough to disrupt early family relationships, and they are put into the mainstream, where they find themselves without peers of the same auditory context. The difficulties deaf children experience when trying to break into a new social 
group can be overwhelming. In fact, all my participants who were mainstreamed, save Amy, seemed, initially, to have been unsuccessful in their ventures to explore the social world provided to them at school.

Forming social bonds with hearing schoolmates can be very difficult for a deaf child whose degree of deafness is significant. The four other participants each told of struggles they faced in elementary and high school with regards to making friends. They used the concepts excluded, isolated and lonely to describe their social contexts. Their auditory contexts are especially relevant here: these participants' hearing loss puts them in categories of moderately severe to profound $(60 \mathrm{~dB}$ or greater).

Communicating with hearing peers poses a great challenge for these participants. Jessie and Steph, whose degrees of deafness are the highest after Ben's, both emphasize feeling excluded, but for different reasons. For Jessie, humour is a central component to the formation of friendships, but her auditory context prevented her from accessing this linguistic element. She had a very clear memory of her experiences with peers; of her attempts at interacting with them, she explained,

[In] kindergarten and all that, I was being excluded. And then... I tried to make friends. It was hard for me to make friends because they sometimes joked to me, or something like that, and I don't know the jokes, I don't know the songs, and I'd get frustrated.

Steph also talked about exclusion, but for slightly different reasons:

Well before, when I was a daydreamer and a troublemaker or whatever, I guess I hung out with people and chatted all the time and whatnot, and then I started focusing on school and I got my hearing aid - I don't know which one caused stuff to change, but anyway... people in my grade started to get nasty... Being excluded was shitty but I could deal with it, no problem.

Based on Steph's description of herself and her social interactions, she seems to have been an introvert, not really making much effort to socialize with her peers. She avoided 
committing to a description of her former self by softening "chatted all the time" with "I guess", which seems to indicate that she was not sure of that situation. Her use of "daydreamer" and "troublemaker" were also not her own descriptors: this is what her teacher had labeled her. Evidently, her increasing hearing loss was causing problems in class, and this seems to have spread to involve her peer relations as well. Her acquisition of a hearing aid may have helped to make her feel more confident and try to connect with those around her, but one could infer that she had not learned the conventions necessary to make friends.

When communication with peers is too much work (for the deaf child or their classmates), the result is a lack of social bonds too. Shayna and Joey used different concepts to describe their early social experiences: isolated and lonely. As Shayna explained,

In elementary school, I didn't socialize with the other children at all. The only socializing that took place was when other kids teased or mocked me. I didn't have friends. I tried to approach other children and befriend them, but eventually they would find that communication was too difficult and they would distance themselves... I was isolated.

Joey described a similar experience:

In school, my peer-relations... I had a couple of friends, through the years, but I wasn't really social. In high school, it was pretty lonely. I think I went weeks without opening my mouth in school, not say anything... and even in my first few years in university... I've never been really social.

What these passages show is that these participants' experiences are defined by missingout on communication. By being placed in a hearing environment, they were forced to grapple with their shortcomings in a challenging and unwelcoming milieu. When contrasted to Ben's experience with school, it stands to reason that segregation in a Deaf community amounts to more social bonds and a clearer sense of self. Shayna, Jessie, 
Steph and Joey were not taught how to interact socially in the hearing world, and due to their auditory contexts, they were not privy to learning by experience, the way hearing people are. The result was exclusion from the hearing community found at school. In addition to exclusion, bullying is another, more severe result of this break in communication. Here, Shayna shared her own experience with bullying:

They stole my money, they would sneak up behind me (I couldn't hear them) and they would pull my bag off my back and run away with it. Yeah, it happened two or three times a week. Yeah, it happened a lot. A few times, they chased me home. It was scary, running away. I was scared. They would throw rocks after me - it was bad!

Bullying was a prominent theme in most of the participants' dialogues. Those who indicated that they had felt excluded and isolated while in school also experienced oppressive behaviours from their peers. Interestingly, most participants who reported being mistreated by their peers explained that this behaviour was unrelated to their hearing loss. Only Shayna blamed her difference for their actions:

I think it was related, yes. I don't know... bullies, they find anything to pick on you for, it doesn't matter what. I was different, deaf, hard-of-hearing, doesn't matter - they picked that, specifically, to focus on and tease me about.

The tendency among my participants was to take responsibility for the bullying, through process of self-blaming attribution (Bauman \& Pero, 2010), but to lay the blame on some aspect of their personality. It is interesting then, that Shayna explained the bullying by pointing to her auditory context as the cause. Her refute could indicate that she was confident in her ability to communicate and therefore refused to accept her part in this situation.

The other three did not exhibit this confidence in their communicative or social abilities. Jessie, Steph and Joey did not charge their differences in hearing with the 
bullying. These three brushed-off their peers' actions as being products of their own social ineptitudes: Joey cited his tendency to be introverted; Jessie ascribed it to her inexperience with jokes; and Steph connected it to the improvement of her study habits.

It is noteworthy that these participants would sooner blame aspects of their personalities than their physiologies. It seems that their need to be audiologically indistinguishable drove them to find traits they could control, even if, in doing so, they were being self-deprecating. In fact, by pinpointing their shortcomings as social beings, they rejected the implications that would otherwise follow: that their situation was unchangeable. Indeed, if their hearing contexts were unimportant, they could become the same as their peers, save a few quirks. These participants sought to show that they were not different from others. They tried not to other themselves.

By controlling the changes necessary to socialize, my participants were able to maintain an identity that allowed for membership with the groups of their choosing. Jones et al. (1984) cite concealability as an important factor affecting stigmatization. My participants seemed to be making an effort to keep their 'handicaps' hidden and even admitting that deafness caused bullying would have been making it visible. After all, deafness is not visible. Jones et al.'s list also cites disruptiveness as an affective factor, explaining that a condition that impedes communication and interaction is also problematic. Here, deafness becomes an issue, as its primary effect is interference with communication.

Communication, by virtue of being the medium through which bonds are created, is essential for all children, so they can take part in communicative events with their peers. Socially, mainstreaming is less than ideal for children whose hearing status puts 
them in the moderately severe to profound ranges because their deafness prevents them from following conversations. Superficially, it would seem uncontroversial that, for these children, education took precedence over socializing ${ }^{6}$. That is, it is natural for parents of deaf children to place them in the mainstream, when to do otherwise would be to go against the societal grain. But children in the mainstream, whose hearing does not allow them to be included in these social activities with relative ease, are at a true disadvantage.

To summarize, relationships between children and parents are formed through interaction and communication. Deaf children of hearing parents are in a difficult situation because communication is compromised due to the child's trouble with hearing and spoken language. This sometimes leads to tension between the deaf child and the other family members.

Lack of communicative practice at home might leave the deaf child ill prepared to socialize with peers upon entering school. If the child enters a residential school for the deaf, they are likely to be surrounded by peers who share their auditory context; if the child enters the mainstream educational system, they are unlikely to be in this situation. When the deaf child enters school, they may seek relationships that can fill the void caused by the absence or deficiency of parental relationships. However, when these children seek out relationships with their peers, these attempts are often unsuccessful. They then experience exclusion, isolation and even bullying.

\footnotetext{
${ }^{6}$ There is a widely held belief among parents of deaf children that education at a residential school is of lower caliber than in the mainstream (Thumann-Prezioso, 2005).
} 


\subsection{Identity and Group Membership}

In this section, I offer an analysis of how the participants see themselves and how they feel that they fit, in relation to the Hearing and the Deaf community. As became evident when I looked at the participants more closely, the concepts of identity and group membership are connected in very complex ways. Despite the seemingly straightforward relationship between identity and group membership, in fact many factors may affect whether choices relating to identity necessarily predict decisions about group membership, and vice versa. In other words, although a person might identify one way, they may nonetheless choose not to belong to the associated group, as I illustrated in my introductory anecdote. And the opposite scenario may be true as well. In addition to this, it does not seem that identity or group membership are directly related to degree of hearing loss, although some aspects of the relationship are fairly transparent. Below are two charts. Chart 1 positions the participants according to their relative degrees of hearing loss. In Chart 2 is a very crude and partial picture of where the participants sit along the Deaf-deaf scale.

Chart 1: Participants' Degrees of Hearing Loss

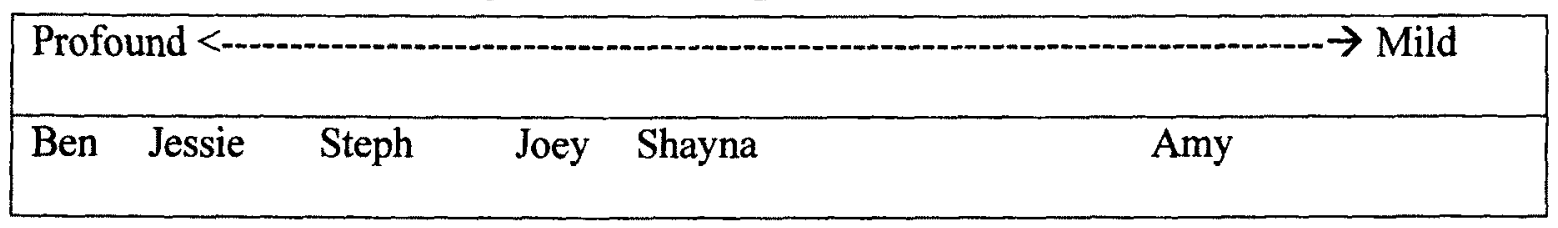

Chart 2: Participants' Social Identity Continuum

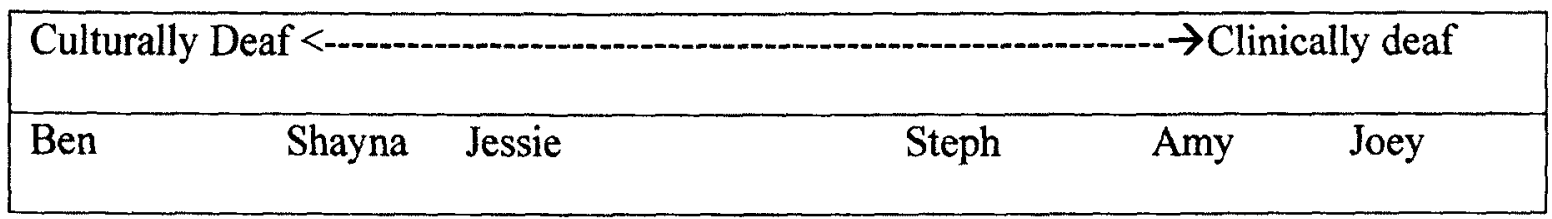


Two participants sit at opposite extremes of this spectrum while the other four fall at different places along the continuum. In actuality, certain characteristics of theirs allow for this representation, but others contradict my placements.

Here, my participants define their identities:

Ben: “[I'm] Deaf"

Jessie: "I do see myself as a deaf person, with cochlear implant... But if I take it out and show my real self, a real human, then I'm deaf. That's me"

Amy: "I have my own place - hard-of-hearing - in the middle"

Steph: "I guess hard-of-hearing". And later in the interview, "obviously, I can't relate to being deaf-deaf"

Joey: "I am hearing impaired"

Shayna: "I identify as Deaf, I feel that more... My mom tells me I'm hard-ofhearing"

Ross (1993), almost 20 years ago, argued that many people who identify as Deaf do so out of necessity, because their residual hearing was not exploited successfully, and so Deaf identity becomes a default identity. He claimed that the proper handling of residual hearing gives deaf persons the opportunity to choose the hearing world as their 'home', and to live well as hard-of-hearing members of society. This can be examined using three of my participants in particular.

Joey maintained that he was a member of the hearing world. He explained that he had had negative encounters with members of the Deaf community, but that more importantly, he felt his personal experiences didn't resemble those of Deaf people. He 
said: "Knowing that literacy levels are very different in the Deaf community", and that... I'm a person who loves to read, to write... I don't really feel a connection or that I would be able to relate. His explanation for why there would not be a connection was somewhat superficial; his love of reading and writing is only one part of who he is. His opinion, it seems, is a result of having been kept so close to the medical community, who reject the possibility that Deaf people can live fulfilling lives while choosing to remain segregated.

Joey would be seen as a "success story" through Ross' (1993) lens. Ross' (1993) definition of success, however, ignores the importance, or the role, of the individual experience. Joey's use of the term "hearing impaired", then, may be problematic. The term is a loaded one: from Ross' (1993) perspective, its use by a deaf person would mean that they accept the hearing world and reject the Deaf world. The act of using a term such as "hearing impairment" connotes that its user frames his identity in terms of what he is not - how he differs from others - and not by what he is. Indeed, the term is hearingcentric, focusing on ideas of lacking and inability. And according to Ross' (1993) definition, to identify as hearing impaired means that the individual's identity is based on shortage and inequality. This is expressed as a tension between who Joey maintains he is and how he sees himself in relation to those around. His sense of self was the most troublesome of the group's, as it revolved around disability, disadvantage and deficiency: [I am] not severely disabled, but I do think that there are some points where I am handicapped in life, I guess... In social situations, it's just much harder for me, in terms of work, if I have to... use a phone, or interact with people with strong accents - I will struggle. In terms of education, it's like with work, it's that much harder for me to make the same progress that it would take a non-deaf person to.

7 It should be noted that, what Joey knows about the Deaf community is "a passing knowledge... basically second-hand knowledge, from the auditory-verbal therapists, who do have a somewhat biased perspective". 
At the other extreme, both Ben and Shayna identified as Deaf; confident in who they are, they know that the Deaf world has more to offer them. Ross' (1993) argument would be that these participants were not properly cared-for socially, while at school, and this seems to have been true for Shayna as well. However, when Ross (1993) claimed that properly exploited residual hearing leads to gained opportunities, he took for granted that the ability to hear correlated with acceptance from others. Both Ben and Shayna were trained to make use of whatever hearing they had and yet neither found that using their hearing was integral to their life. Rather, they preferred the idea of the Deaf world, where acceptance is not dependent on the ability to hear.

Identifying as deaf and joining the Deaf world are two different things. Ben is an example of someone who identified as Deaf and was predominantly immersed in Deaf culture. He reported being firmly rooted in the Deaf community. He stated that, in the Deaf world, he had access. In the hearing world, he said, "I don't know [how it works], I have to learn to get around... I'd have to have my identity explained to me, communication is convoluted, it's bothersome. So I keep it superficial". Ben was candid about the effort that is required for a deaf person to assimilate into the majority. But by criticizing the value of communicating within the hearing world, he seemed ready to relinquish his membership with the hearing world. His connections to the hearing world are nonetheless present (his family is hearing and so is his workplace), but they are generally less meaningful than his affiliation to the Deaf world, which has given him the ability to conceive of his context as healthy. As Ben explained,

I don't label myself as disabled; I'm deaf, that's it. Most people look at us and think we are [disabled], but I can't hear - that's all. It's no big deal. I can sign deaf are more focused on the visual than the auditory... [but] it's not that complicated to talk to hearing people, you don't need a science degree for that. 
Shayna is an example of someone who reported identifying as Deaf but was not a full member of the Deaf community. Her feelings of group membership were similar to Ben's but more tempered. She claimed that she wanted to be part of the Deaf world and was working towards that goal, but still had unavoidable and complicated ties to the hearing world. She explained,

I was always trying to make my mother proud, she always gives me the feeling that being deaf or accepting my hearing loss would influence my life negatively. I went along with it to try to make her happy. Now I feel that I'm closer to the Deaf world... I'd prefer to be part of the Deaf community, to join it... but it's hard. My mother doesn't accept it, so... I always feel a bit disjointed, separate from her.

For Shayna, it seems that joining the Deaf community means rejecting membership with the hearing world, as with Ben. Interestingly, although Joey, Ben and Shayna see the choice of claiming membership to one world as mutually exclusive, this was not the case for all the participants.

For deaf persons, the creation of multiple identities is a very likely scenario. This might happen in order for them to come to terms with often opposite and contradictory parts of themselves, especially as multilinguals. The multitude of possibilities available to them may cause them to create a number of identities to reconcile these internal, or external, struggles. For example, they may form different identities that relate to the dynamics of the relationships they may have with hearing people, and identities that relate to the dynamics of those they have with deaf people.

Jessie is an example of someone who claimed to feel comfortable with balancing both memberships and both identities. She disclosed feeling more comfortable in the hearing world, for now, but at the time of the interview, she had spent less time with the Deaf community than Shayna. Of what world she belongs to, Jessie said, 
I would say both - if I'm allowed - yeah, neutral. In the middle, I could say. ...before I was more into the hearing world, I start to see how people react, their expressions, how they talk in a conversation, how they are - laid back or stiff, or whatever - then I know how to accept it.

As Jessie made clear, there is an intentional learning process when it comes to joining a foreign community, and joining this foreign (hearing) community is something she has worked hard at since childhood. And this process occurs in any instance of wanting to join some community. But her explanation of where she fits was not as straightforward as she made it seem: neutrality is not a state of accepting multiple options; rather it is a state of accepting neither. Perhaps she gave the impression of being comfortable with having membership with both because she actually is a member of neither one.

Well I have this technology that can help out so it doesn't matter anymore. It's not a disability, it's just one small thing. It doesn't affect me at all... well, yeah, it affects me, because I can't hear - if I take it off - but still, it doesn't matter. So it's not considered a disability - it shouldn't be... A disability is something that... it prevents - umm no, I can't say prevent - umm, something that you can't do but still you can work on it, and work around it, and still be functional.

For Jessie and Shayna, making a decision about where they fit was more difficult. Despite the fact that both struggled with their involvement in the hearing world, it does not seem, as Ross (1993) suggests, that their movement towards a Deaf identity is due to failure in the former. Indeed, their movement is gradual and deliberate, but not coloured by distaste for the hearing world. Rather, they seem to be attempting to reconcile their feelings of either-or. Both showed that they straddle both worlds and both seemed to need to do so. Ross' (1993) claims overlook the fact that identities are evolutionary in nature, constantly building and transforming. 
If we adopt McCracken's (2008) idea that a vertical status continuum exists, along which people strive to improve their placement, we can see that, when Jessie and Shayna moved from one community to the other, they re-negotiated their place in society, and along that continuum. In order to be able to swing from one world to the other, these participants first had to negotiate their memberships with each community, and build an identity that fit each of these communities. This is something both were still in the process of doing when they were interviewed.

This concern for selecting one identity over another was experienced by Steph as well, although somewhat differently. She seemed to feel that she had a definite connection to the Deaf community and Sign Language, but also felt that she fit in better with the hearing world. She also included her hearing loss in her list of identifying characteristics, but not in the way Carty (1994) proposes, that is as a sign of Deaf identity.

Steph appeared to conceive of identity and group membership in an opposite way to the previous participants. For her, the act of proclaiming membership with the Deaf community would have a significant impact on her identity, not the other way around, as the others indicated. She claimed to be part of the hearing world, but mostly because she had grown up there. She seemed confused about what her involvement in the Deaf community would mean:

I've lived my whole life in the hearing community, so I know how hearing people think and I think... along the same lines, right? I mean, you go to school, you get a job, you get married, you have kids... I never thought of different things, like it's the path I know; it's the path I grew up being taught.

As Burke and Stets (2009) explain, the experiences Steph lived in the hearing world have resulted in this image of what it means to be hearing and conversely, how 
being Deaf would change that. Her understanding of identity and membership, in this context, is somewhat binary, and yet, she appeared to be able to juggle multiple identities when they are unrelated to her hearing status: engineering student, ASL student (because for her, learning ASL was initially unrelated to her auditory context), and rugby player, to name a few.

How the individual identifies is closely tied to bonds that are formed between the individual and others. For some, it seems that encouraging 'normalcy' rather than individual difference and seeking to assimilate rather than segregate allowed them to adopt a sense of identity focused on their similarities to the hearing world and not their alterity to it. Below, I present an analysis of how my participants seemed to respond to social pressures related to their auditory contexts.

\subsection{Changes in Auditory Context}

The difficulties deaf children face when trying to form relationships with their hearing peers may have a significant impact on their identity and group membership choices. According to my participants, there were two main responses to the isolation they experienced while at school: they could opt to change their person or they could opt to make an adjustment to their social surroundings. Both these options included the finetuning of language and communication skills, but this fine-tuning may nonetheless have taken them in different directions. They could move towards technology, spoken language and the hearing world, or they could move towards Sign Language and the Deaf community. As we saw above, for some, the choice between the two is not easy, nor is choosing one necessarily exclusive of the other. 
First, then, an account of how language and communication affect their social relations is presented, with examples. Degree of deafness affects communication skills, which translates into difficult social relations, both with parents and with peers. When my participants became aware of this challenge, they addressed the issue by turning their attention to their communication skills.

As was shown, hearing-deaf friendships were difficult to form initially, but what's more is that they were also difficult to maintain. As previously described, Urberg, Degirmencioglu and Tolson (1998) describe friendship as something that forms out of similarity. As individuals grappling with who they are, and who they socialize with, my participants seemed to swing back and forth often. This put strain on existing relationships. Some of my participants expressed that they had struggled to maintain friendships with peers who did not share their auditory contexts, and therefore, their experiences. Here Shayna recounted one of her experiences:

The two of us are best friends, really close. Recently she told me it's hard to communicate with me now. If she wants to call me, she can't because I don't answer, so it's hard. I told her, yeah, text me. She says it's expensive, she can't... So, I say, ok, email me. She's like, 'email?!' (rolls eyes). Ok, Facebook? Hmmm, hmmm... (exasperated gesture). She's not trying, so I feel like she doesn't want to try and I can't force her, it's up to her.

When one, or both people change, the relationship may suffer, as Shayna's anecdote illustrates. Tillmann-Healy (2006) asserted that if the friendship was initially based on similarities in attitude and values, and these changed over time, the relationship would cease to be fulfilling and neither person would continue to be validated. Because of Shayna's shift in identity, she is losing friends. At the same time, the loss of this friendship results in a change to Shayna's group membership preference. Unsurprisingly, she reported that relationships in the Deaf community were easier to form and maintain. 
A shift in identity encourages a person to seek out other social opportunities.

Jessie's experience was that her cochlear implant provided her with another community, one made up of other implanted deaf persons. Here, she described meeting others like her:

I noticed one or two people had cochlear implant, and they went through almost the same thing as me. And it's like finding someone on a lost island, someone who's same like me, and we are able to share the experience and... the other person has different opinion, but it's right, then I would say, oh that's true, then we can understand each other more and understand what the situation really is, then you start to build your knowledge on it, you start to build your self-esteem, your confidence and you realize, ok, that's the world, how it is.

New social groups - new communicative opportunities - provide new information about the world, which is used to build identity (Burke and Stets, 2009). Jessie talked about her world knowledge as something collaborative, as a collection that grows and expands when she comes into contact with another person. As Smuts (1927) put it:

I would never come to know myself and be conscious of my separate individual identity were is not that I become aware of others like me: consciousness of other selves is necessary for consciousness of self or self-consciousness... it is through the use of the purely social instrument of language that I rise above the mere immediacy of experience and immersion in the current of my experience. (as cited in Joseph, 2004, p. 8)

This all comes back to language: without it, one cannot create bonds with others. For a deaf person attempting to join the hearing community, this is the disability; not the fact of hearing differently. Knowledge of the world and one's identity is collaborative and people learn about themselves through bonds with others. This process leads to an understanding about where they fit into the world, but for deaf people, communication through a spoken language is difficult and therefore, their understanding of the hearing world is incomplete. 
This can be remedied by an intentional improvement in communication skills.

Jessie talked about her difficulties with communication proper, in particular her difficulty with humour: "[It] was difficult because... they pick on me, so I pretty much can't do anything until I know how to make jokes, until I know how to fight back, I guess". For Jessie, the ability to use humour is a weapon and a tool she could use to enter this group of hearing peers. As McCracken (2008) explains, joining a separate group requires the perfecting of conversational skills. Jessie's anecdote illustrates this perfectly; she was explicit about the role informal language played in her feelings of transformation. During a trip to her native country, similar-aged family members "taught" her how to use witty language and how to make jokes. She said,

In grade 12, I started to learn more about jokes, I started to watch more TV shows and I started to catch some things. And when I watch TV shows, there's closed captioning, which helps me the most... because with more understanding of the language, with more understanding of what that situation is talking about, then I can be able to relate it to my situation nowadays, then I can say the jokes too... and then my brother, tells me, if you're in that situation, you have to have the right time of saying it; like if you say that joke in a different time, that's stupid.

Communication skills are vital to the formation of bonds for the purposes of group membership. According to McCracken's (2008) suggested hierarchical continuum, Jessie made a concerted effort to move vertically, towards a better-situated group. This continuum is problematic for these purposes, however, because it assumes that there are two extremes: deaf at the bottom and hearing at the top. In fact, these extremes are impossible, as a deaf person can never be a hearing person, no matter how hard they try, or what technologies they use. As a society, we conceive of the continuum in this way in part because we want deaf people to move upwards, hence our insistence on their use of 
amplification devices. Still, for McCracken (2008) to suggest that the perfecting of conversational skills is enough to move up on the continuum is incomplete.

McCracken's (2008) group membership continuum must be reworked for my participants' purposes. For them, the continuum is V-shaped and the bottom refers to deaf people. These are people who are disabled in both the hearing and the Deaf world. One of the upper ends of the $\mathrm{V}$ designates hard-of-hearing individuals, as defined by Sorkin (1996); the other side identifies Deaf people. My participants either move up towards one end or the other; a few move up in a vertical line from the bottom, to a place that floats between the two upper ends. When the situation dictates that they act Deaf, they might keep their hearing aid on, but they will use ASL in conversations. When they are in a situation that demands that they behave as hearing people, they move towards the hardof-hearing extreme, using their hearing aid, and communicative and social knowledge.

In order to move towards one group or the other, then, one must focus on language to be able to communicate and form social connections with members of that group. But language is not the only factor in group membership. For those who want to join the hearing world, but are disadvantaged by their auditory contexts, they might choose to use technology to change their situations.

Outspoken Deaf personalities in the media tend to propagate the idea that Deaf people are proud of who they are and have no desire to hear, but my participants did not all appear to subscribe to this way of thinking. In fact, only Ben described himself as Deaf and did not express a desire to change. He was also the only one to go without any hearing amplification devices. All the other participants used some sort of technology. In itself, this seems to indicate that they disagreed with the claim that deaf people are 
content as they are. On the other hand, the participants who used hearing aids expressed that they would not want to further change their auditory contexts. In their cases, Jessie and Joey were content with having just the one implant; Jessie was satisfied with her single CI, while Joey still used a hearing aid in his non-implanted ear.

Shayna, who claimed she wanted to join the Deaf community, displayed an ambivalent view of amplification devices. Sometimes, the attitude she displayed in her interview seemed negative towards the technology, claiming she preferred not to wear her hearing aids and that she would never implant her child. At other times, she took it for granted that hearing aids are a natural part of deaf people's lives. Interestingly, these contradictory sentiments sometimes showed up together:

Often hearing people feel that cochlear implants are the best answer, that every [deaf] child should be implanted. I disagree. My - well, now my boyfriend, his name is [Adam], he's deaf, he wears hearing aids in both ears - his family is the same as mine. They both think 'hearing'... [Adam] prefers Deaf identity, he's a proud Deaf man... we would never get implanted with CIs. We feel the same on that - it's good.

The contrast in her stance demonstrates two of the identities she embodies. When she said that she disagreed with hearing people about the value of cochlear implants, this was a manifestation of her Deaf identity. It is a value of the Deaf community that cochlear implants are poisonous to both the person and the culture, and Shayna has adopted this value in the process of her transformation. But her own life experience was inconsistent with this value, as hers included the use of hearing aids, and as much as she claimed to dislike her hearing aids, she continued to use them and even assigned them a part in her successes. For Shayna, as much as she opposed the 'correction' of deafness, she accepted the enhancement of the ear as unremarkable. She immediately followed her condemnation of CIs by associating deafness and technology, when she explained that 
her boyfriend was deaf and wore hearing aids. Shayna views hearing aids and cochlear implants as very different, yet their effects on identity are not drastically different.

Perhaps the finality of the cochlear implant is what some deaf people feel uneasy with: a hearing aid can always be removed and therefore may not be seen as a device that alters a person's identity in a fundamental way. The cochlear implant, on the other hand, cannot be removed, and becomes part of the human in a much more intrusive way. The cochlear implant, then, is a symbol: implanted deaf persons can never reject that very small hearing part of themselves. For those who have a $\mathrm{CI}$, however, this symbol is unobtrusive. Jessie described her $\mathrm{CI}$ as indispensible:

If my battery dies, [I take it off]. Or I forgot my battery, I take it out ${ }^{8}$. But if it's in a situation where I have to hear, like say I have a class to go to and my battery dies and I don't have [another one] with me, then I can't do anything, I'm stuck.

Jessie is apparently entirely dependent on her implant to help her cope in situations involving hearing peers. In this instance, the only difference between Jessie and Shayna is the implant. If Jessie didn't have a CI, she might be better able to transition between both worlds, instead of being "neutral" about where she fits. So it seems cochlear implants do not allow the wearers to drop the hearing part of themselves. Rather, because the Cl's become one with each person, they stand in the way of that person really exploring other possibilities. In Shayna's case the technology is removable, which allows her to be Deaf. She has the luxury of deciding who she wants to be in a given situation. But CIs do not allow this. With hearing aids, there's agency and choice; with CIs there is no agency, no

\footnotetext{
${ }^{8}$ Here, Jessie means that she removes the external part, simply because without the battery, it cannot transmit auditory information to the internal component. Therefore, in this situation, the $\mathrm{Cl}$ can be seen to be 'turned off', but the internal component is still inside and ready to receive information. The ambiguity of her comment is due to her struggles with English.
} 
choice. Even by removing the external part, the implanted is nonetheless hooked in, which creates a psychological dependency as well. It makes the wearer feel as though they need the link to the hearing world at all times, as Jessie's explanation makes clear.

Amy is very much linked to the hearing world as well; her hearing aid was a central issue during our interview. Given Amy's view that she is missing something, it is unsurprising that she focused on technology as a means to move towards 'normalcy'. Yet her hearing aid was also the catalyst for changes to her identity: "I didn't identify as, like, having a real hearing problem until I had that hearing aid". Her acute awareness of the stigma attached to amplification devices led her to stop wearing hers during high school. For Amy, wearing a hearing aid meant that she was conceding that something was wrong with her: "[it felt like they were saying] we can't fix you with surgery and you're not good enough the way you are, so we're gonna give you this little tool that's gonna help you hear so you can be like everyone else in your class".

Amy's hearing status had been a challenge for her, but at the time of the interview, she had a very advanced hearing aid that was equipped with Bluetooth ${ }^{\circledR}$ technology. She described using it: "I can be on the phone [through the hearing aid], but still be so much aware of everything else that's going on around me". What she described was a situation that many of us with 'normal' hearing do not often experience. In fact, with the help of this device, her hearing is so advanced that it competes with many mainstream cellular devices. Amy's experience illustrates Featherstone and Burrows' (2005) assertion: "the machine parts become replacements... to enhance the body's power potential" (p. 174). Oftentimes, hearing aids and cochlear implants are used to elevate the wearer's hearing to a level that resembles that of the greater population but, in 
this instance, Amy was encountering a situation in which she was even better positioned than the general public. "If I had the [hearing aid] I have now, back then, I'd probably be like, 'check it out! It has Bluetooth $囚 !$ ' It'd be so much cooler".

Jones et al.'s (1984) theory concerning stigma comes up again as Amy acted in a way that contradicted what the researchers claimed. Concealability was not a negative factor for her; rather, she drew attention to her hearing aid, and by extension, her hearing status. In fact, by taking pride in her physical hardships and in the consequences of her surgeries, and by boasting about her opportunity to use a very advanced technological device, Amy re-appropriated her body as technological, where the boundary between organic and inorganic was blurred. As Shilling (2005) contends, technologies are very powerful entities in that they become one with the society in which they exist. They take root in the norms and practices of societal structures, especially those structures that become part of its members' actions and identities. Further, those who choose to make use of them tend to be the younger generations. Indeed Amy - one of the younger participants - exemplified Shilling's explanation perfectly. She not only came to accept her hearing aid, she revered it.

Featherstone and Burrows' (quoted in Shilling, 2005) explanation of technology as enhancing tools applies to the anecdotes I have just presented. From this angle, hearing amplification devices can be seen as tools that provide the individual a way towards upward mobility.

In using technology to restore previous bodily capacities and the options that social environments may once have offered, but now no longer seem to, there is a sense in which the substitutive effects of this resource also extend and enhance the physical body and its environment. These supplementary uses of technology have long been significant in terms of how people are positioned within society. (Shilling, 2005, p. 188) 
Certainly, Shayna, Steph, Joey, Jessie and, especially, Amy are examples of how this can happen in actuality.

The use of technology to transform oneself to mimic the hearing majority, however, is not necessary. As mentioned earlier, the idea that our social continuum is binary does not take into account the Deaf community (or any other community on the fringes of mainstream society). It does not leave room for alternative conceptions of success but rather assumes that upward movement involves a higher paying, and therefore higher status job. To define success in these terms is to overlook all the other factors that many people aspire to (for example, satisfaction and happiness, family, and friends, to name a few).

Only Ben had a clear-cut opinion of himself and his auditory context. He was the only one who was a member of the Deaf community and who signed fluently. While the other participants talked about the hard work involved in overcoming deafness, Ben did not express a need to overcome his auditory context. As he apparently saw it, deafness is a disability only when he is required to do something 'hearing', such as use a phone or listen to speech - essentially, anytime that he is made to hear and use a spoken language, especially receptively. Instead Ben embraced his deafness, as Carty (1994) observed, and the modality that suits his strengths best: visual.

Hearing amplification devices may help the wearer to hear sounds better than without them. This fact, on its own, is not enough for a deaf person to manage in a hearing community; users of hearing technology must also learn to better communicate in the dominant spoken language. And although some of my participants claimed not to be interested in changing, the act of wearing a hearing aid does change their auditory 
contexts. As we just saw, hearing technology also has significant effects on how the wearers see themselves and the world, and how they feel the world sees them. Shayna described:

I noticed a machine, I didn't know what, maybe a fan or something. So I was looking around for the noise and my mom cried. She cried, because I could hear. Say there was a noise or a loud sound and I started to hear, she cried, 'oh, you can hear!' My father just said, 'good, fine'.

Hearing devices go hand-in-hand with aural-oral communication. All the participants who made use of technological hearing devices were primarily spoken language users. They all spoke the majority of the time and two of them did not report using Sign Language at all. Joey is dependent on his hearing devices for all activities and explained that he had no desire to learn to sign. At the time of the interview, Amy said she would use ASL if the situation required it. She expressed a contradiction, however, by stating that she felt that she could become part of the Deaf community if she signed more and took part in more social activities. Still, she does not socialize with other Deaf people regularly. At the other end of the spectrum, Ben, the only participant who did not use any type of hearing device, was firmly rooted in the Deaf world and preferred to use ASL as much as possible.

But as mentioned previously, identity and group membership do not always coincide, as identity is an oscillating concept. It is those participants who split their identities between being Deaf and being hard-of-hearing who had the most to share. Jessie's cochlear implant allowed her to float between the hearing world and the Deaf world. In the hearing world, she said she was able to take part in typical everyday activities: family, school and social interactions are all part of her daily life. Her cochlear implant does not play a role in her interactions within the Deaf community - interactions 
that she maintains on a regular basis through her partner, and Deaf friends and acquaintances - though she nonetheless continues to wear her CI while interacting in the Deaf community.

Balancing a Deaf and a hard-of-hearing identity is something Jessie seemed to pride herself in doing. Her partner, John, does not wear a hearing aid and he does not speak very often. He was raised to be Deaf, going to a residential school and having many Deaf relatives. During the interview, Jessie expressed that she would like her partner to practice speaking and hearing (to put his aid back on). This seemed to indicate that what she valued most was being able to negotiate both identities equally. She even described acting as the interpreter between her partner and her parents, translating what each one tries to communicate to the other.

Interestingly, as much as she reported liking being able to balance both identities, she put much more importance on spoken language, and by extension, the ability to hear and the hearing world. For Jessie, if John wore his hearing aid, he would be able to speak for himself. In her family, as Sign Language is not valued, lacking a voice is akin to lacking agency. Her apparent view of ASL reflected this as well: "I prefer speaking because I can always say [exactly what I mean]. Like if I talked in Sign Language, it's very general... but with English, you have specific... questions... that lead to specific answers". (Even her word choice - "talk" - to describe the use of Sign Language is meaningful).

Wearing hearing technology does not necessarily mean that the wearer is committed to the hearing world, however. Shayna wears her hearing aids most of the time, yet she yearns to commit more fully to the Deaf community: 
Since I've started, Sign Language has felt more natural, it feels natural for me, I prefer it. I can speak, my voice is clear enough, I can speak and communicate fine, and I can hear your voice if I wear my hearing aid. I always wear my hearing aid (in most of my classes), I prefer not to have it on, like right now, I'm not wearing it. So Sign Language is perfect. In the future, my language of preference will be sign, I know it.

This passage illustrates Shayna's back and forth between identifying as a Deaf person and identifying as someone who is hard-of-hearing. On the one hand, she appeared to subscribe to fundamental Deaf values by proclaiming that Sign Language was natural for her - more so than speech - and that it would become her dominant language eventually. On the other hand, she seemed to pride herself on her 'hearing' skills: clear speech, her ability to hear and understand when someone speaks to her.

Both identities were true and necessary for different communicative purposes and neither Jessie, nor Shayna, could say absolutely who they are more. It is clear from this dichotomy that both participants esteemed both sides of themselves and that these are part of two distinct and opposing identities. It is specifically what Pittam (1999) describes when he says that people may hold contrary positions regarding their own identities "even across the space of a single interaction" (p. 116).

When they feel isolated, then, deaf people may decide to change an aspect of their world, be it themselves or their environment (i.e. the community to which they belong). These decisions involve considerations of the self and of social circles. When they opt to become more rooted in the hearing world, they are forced to make use of hearing amplification devices and improve their communication skills in a spoken language. When they opt to become more involved in the Deaf community, they must learn to express themselves in Sign Language. For some, these options are not mutually exclusive, but they are difficult to balance, as the relationship between identity and group 
membership is one riddled with complexities. And their decisions about group membership involve language and communication, and technology, which further complicate the issue.

\subsection{Summary}

In this chapter, I presented the results of my research. I began by focusing on my participants and their social relations. This included both a characterization of their relationships with family members and then an account of their experiences with friendships. By their accounts, it seemed that most of my participants were challenged when it came to forming meaningful friendships with hearing peers at school.

The next section of this chapter discussed identity and group membership. Here, it was evident that the relationship between these two concepts is extremely complex. Even attempting to marry the two topics for the purposes of the chapter proved to be a difficult job. This discussion centred on my participants' identities and their affiliations with the majority and minority worlds at their disposal, as the participants saw them.

Unexpectedly, this was much more difficult because the relationships between how my participants self-identified and their group memberships did not necessarily coincide. I believe this difficulty is an analogy for the challenges my participants face on a daily basis, when they too, try to reconcile the two concepts. While some were able to pronounce clearly who they were and how they felt they fit into this world, others instead were stuck in limbo, teeter-tottering between multiple and opposed identities and memberships.

In the final part of this chapter, I presented the options my participants grappled with, in terms of improving their situations. Some chose to move towards the Deaf world 
while others chose to put more effort into joining the hearing majority. This section shed some light on the processes involved with group membership and provided insight as to how my participants conceptualized of their contexts. There was also a significant emphasis on technology and its role in the construction of identity. It seemed that, although language is an important indicator of identity, so is technology for most of this group of individuals. 


\section{Conclusion}

In this thesis, I have presented the social histories of six deaf individuals in an effort to shed some light on the processes of identity construction among this population. In order to discover more about what plays a part in identity, I used a framework of concepts that included looking at language learning and use; social relations at home and at school; and technology. Above all, the most prominent aspect of their stories has been the uniqueness of each one's identity formation, despite the similarities of their contexts.

Their family backgrounds were all similar and their passages through school all started in the mainstream. The majority of my participants experienced difficulties when it came to building and maintaining friendships, and these seemed to be due to communication barriers. In order to keep up, most of my participants used technology to support their efforts at improving their hearing/speaking skills. Although these efforts were made, socially, my participants struggled to get close to their hearing peers. This is the extent of their similarities, however, and how they internalized these experiences is unique to each one.

Difficulties with peers and the use of technology were important factors when it came to choosing how they saw themselves and which groups they chose to associate their beings with. It seems that language was not always a leading factor in the identity construction of my participants, especially when technology was present. To be clear, language plays a part in identity construction, certainly, but language alone does not seem to dictate the way in which identity is formed. Most of my participants were comfortable using spoken language, and so the role of language cannot be discounted, but its role was not what I had expected. The interplay between the roles of language and those of family 
background, of auditory context, of technology, and of social experiences, is what seems to lead to the creation of identity. Not any single constituent.

As to whether being educated in a modality that suits their strengths will help deaf children have a clearer sense of self, a conclusion cannot be drawn from this sample. On the one hand, it seemed that a deaf education from a residential school for the deaf did lead to a strong sense of community as well as a clear sense of identity. But this was only the case for one of my participants. On the other hand, although the identities of my other participants varied, some who had been educated in the mainstream system did have clear identities, balancing their "hearing" and their deaf sides. Some did not.

As presented in the previous chapter, most of my participants had a difficult time conceiving of identity as a multiplex phenomenon. They seemed not to recognize that their identities changed based on social situations. Somehow, their contexts did not allow them to embrace both, or all, their potential selves because this might have entailed the choosing of a community as the only one to which they belong. Indeed, for some, deciding on an identity meant choosing to unite with one world, and this, in turn, meant renouncing another world. For Jessie, Steph and Shayna - those most indecisive about identity and group membership - the task of reconciling the inherent relationship between these two things was difficult.

There were some limitations in this study. It would also have been interesting to recruit deaf participants whose parents were also deaf. Based on my experience with the Deaf community, my expectation would be that these participants would have had all their schooling take place in a residential school for the deaf, and that their cultures 
would have been passed down from within the family. I would also expect that difficulties with peers in school would be of a different tone.

In terms of sample size, although mine fit this study, and the range of educational and auditory contexts was represented, it would have been interesting to interview a larger sample. My suspicion is that a larger sample would only result in more variations on the possibilities of identity combinations, yet that would be valuable information nonetheless, and could further inform the study of language and identity. 


\section{References}

Able Hearing, Hearing Specialists. (2010). Retrieved Aug. 18-20, 2011, from http://www.ablehearing.com.au/about_hearing.html

Accessibility for Ontarians with Disabilities Act. (2005). Retrieved Aug. 13, 2010, from http://www.e-laws.gov.on.ca/html/statutes/english/elaws_statutes_05all_e.htm

Ainsworth, M. D. S., \& Wittig, B. A. (1969). Attachment and exploratory behavior of one yearolds in a strange situation. In B. M. Foss (Ed.), Determinants of infant behavior, Volume 4 (pp. 111-136). London, UK: Methuen.

Ainsworth, M. D. S. (1979). Attachment as related to mother-infant interaction. Advances in the Study of Behavior, 9, 2-52.

American Speech-Language-Hearing Association (1997-2012). Retrieved Aug. 18, 2011, from http://www.asha.org/public/hearing/

Baker, C. (1999). Sign language and the deaf community. In J. A. Fishman (Ed.), Handbook of language and ethnic identity (pp. 122-139). New York, NY: Oxford University Press.

Baker-Shenk, C., \& Cokely, D. (1980). American sign language: A teacher's resource text on grammar and culture. Washington, DC: Gallaudet University Press.

Bauman, S., \& Pero, H. (2011). Bullying and cyberbullying among deaf students and their hearing peers: An exploratory study. Journal of Deaf Studies and Deaf Education, 16(2), 236-253.

Bourdieu, P. (1991). Language and symbolic power. Cambridge, MA: Harvard University Press.

Bowlby, J. (1969/1982). Attachment and loss: Vol. 1. Attachment. New York, NY: Basic Books.

Bowlby, J. (1973). Attachment and loss: Vol. 2. Separation. New York, NY: Basic Books.

Brennan, S., Gombac, I., \& Sleightholm, M. (2009). Participations and activity limitation survey 2006: Facts on hearing limitations. Ottawa, ON: Statistics Canada, Catalogue no. 89-628-X.

Burke, P. J., \& Stets, J. E. (2009). Identity theory. Oxford, UK: Oxford University Press.

Carty, B. (1994). The development of deaf identity. In C. J. Erting, R. C. Johnson, D. Smith, \& B. Snider (Eds.), The deaf way: Perspectives from the international conference on deaf culture (pp. 40-43). Washington DC: Gallaudet University Press. 
Christiansen, J. (with Spencer, P.) (2002). History of cochlear implants. In J. Christiansen \& I. Leigh (Eds.), Cochlear implants in children: Ethics and choices (pp. 15-44). Washington, DC: Gallaudet University Press.

Colletti, L., Carner, M., \& Colletti, V. (2006, March). Auditory outcomes in children with cochlear implant aged less than 12 months. $8^{\text {th }}$ European Symposium on Paediatric Cochlear Implantation, Venice, Italy.

Creswell, J. W. (1997). Qualitative inquiry and research design: Choosing among 5 traditions. London, UK: Sage Publications.

Cummins, J. (1984). The minority language child. In S. Shapson, \& V. D'Oyley (Eds.), Bilingual and multicultural education: Canadian perpectives (pp. 71-92). Clevedon, UK: Multilingual Matters Ltd.

Cummins, J., \& Danesi, M. (1990). Heritage languages: The development and denial of Canada's linguistic resource. Montreal, QC: La maitresse d'école inc.

Economides, M., Parpounas, K., Koudoumnakis, E., Vagis, G., Choulakis, M., \& Economides, J. (2006, March). Language progress in children operated for cochlear implantation at a young age. $8^{\text {th }}$ European Symposium on Paediatric Cochlear Implantation, Venice, Italy.

Edwards, J. (1999). Refining our understanding of language attitudes. Journal of Language and Social Psychology, 18(1), 101-110.

Edwards, J. (2009). Language and identity: An introduction. Cambridge, UK: Cambridge University Press.

Estabrooks, W. (1998). Cochlear implants for kids. Washington DC: Alexander Graham Bell Association for the Deaf.

Foddy, M., \& Kashima, Y. (2002). Self and identity: What is the conception of the person assumed in the current literature? In Y. Kashima, M. Foddy, \& M. Platow, (Eds), Self and identity: Personal, social, and symbolic (pp. 3-25). Mahwah, NJ : Lawrence Erlbaum Associates, Inc., Publishers.

Freel, B. L., Clark, M. D., Anderson, M. L., Gilbert, G. L., Musyoka, M. M., \& Hauser, P. C. (2011). Deaf individuals' bilingual abilities: American Sign Language proficiency, reading skills, and family characteristics. Psychology, 2(1), 18-23.

Freeman Field, R. (2008). Identity, community and power in bilingual education. In J. Cummins, \& N. H. Hornberger, (Eds), Encyclopedia of language and education, $2^{\text {nd }}$ Edition, Volume 5: Bilingual education (pp. 77-89). New York, NY: Springer Science+Business Media LLC.

Gerring, J. (2007). Case study research: Principles and practices. Cambridge, NY: Cambridge University Press. 
Gesser, A. (2007). Learning about hearing people in the land of the deaf: An ethnographic account. Sign Language Studies, 7(3), 269-283.

Gibson, H., Small, A., \& Mason, D. (1997). Deaf bilingual bicultural education. In J. Cummins, \& D. Corson, (Eds.), Encyclopedia of language and education, Volume 5: Bilingual education (pp. 231-240). Boston, MA: Kluwer Academic Publishers.

Gravel, J. S., \& O'Gara, J. (2003). Communication options for children with hearing loss. Mental Retardation and Developmental Disabilities Research Reviews, 9, 243-251.

Greenwald, B. H. (2007). Taking stock: Alexander Graham Bell and eugenics, 18831922. In J. V. Van Cleve (Ed.), The Deaf history reader (pp. 136-152). Washington, DC: Gallaudet University Press.

Grosjean, F. (2001). The right of the deaf child to grow up bilingual. Sign Language Studies, 1(2), 110-114.

Grosjean, F. (2010). Bilingualism, biculturalism, and deafness. International Journal of Bilingual Education and Bilingualism, 13(2), 133-145.

Haraway, D. J. (2008). A Cyborg manifesto: Science, technology, and socialist feminism in the late twentieth century. In C. Malacrida \& J. Low (Eds.), Sociology of the body: A reader (pp. 354-359). Oxford, UK: Oxford University Press.

Hermans, D., Ormel, E., Knoors, \& Verhoeven, L. (2008). The relationship between reading and signing skills of deaf children in bilingual education programs. Journal of Deaf Studies and Deaf Education, 13(4), 518-530.

Hintermair, M. (2006). Parental resources, parental stress, and socioemotional development of deaf and hard of hearing children. Journal of Deaf Studies and Deaf Education, 11(4), 493-513.

Howe, D. (2011). Attachment across the lifecourse: A brief introduction. New York, NY: Palgrave MacMillan.

Hyde, M., \& Punch, R. (2011). The modes of communication used by children with cochlear implants and the role of sign in their lives. American Annals of the Deaf, $155(5), 535-550$.

Jolliffe, D., \& Farrington, D. P. (2010). Is low empathy related to bullying after controlling for individual and social background variables? Journal of Adolescence, $x x x, 1-13$.

Jones, E. E., Farina, A., Hastorf, A. H., Markus, H., Miller, D. T., Scott, R. A., \& French, R. S. (1984). Social stigma: The psychology of marked relationships. New York, NY: W.H. Freeman and Company.

Joseph, J. E. (2004). Language and identity: National, ethnic, religious. New York, NY: Palgrave MacMillan. 
Kannapell, B. (1994). Deaf identity: An American prespective. In C. J. Erting, R. C. Johnson, D. Smith, \& B. Snider (Eds.), The deaf way: Perspectives from the international conference on deaf culture (pp. 44-48). Washington DC: Gallaudet University Press.

Lane, H. (2002). The mask of benevolence. San Diego, CA: DawnSignPress.

Lane, H., \& Bahan, B. (1998). Ethics of cochlear implants in young children: A review and reply from the deaf-world perspective. American Academy of OtolaryngologyHead and Neck Surgery, 119(4), 297-313.

Lane, H., Hoffmeister, R., \& Bahan, B. (1996). A journey into the deaf-world. San Diego, CA: DawnSignPress.

Lang, H. G. (2003). Perspectives on the history of deaf education. In M. Marschark \& P. E. Spencer (Eds.), Oxford handbook of deaf studies, language and education (pp. 920). New York, NY: Oxford University Press.

Limaye, S. (2004). Exploring the impact of hearing impairment on self-concept. International Journal for the Advancement of Counselling, 26(4), 369-374.

Makabe, T. (2005). Intermarriage: Dream becomes reality for a visible minority? Canadian Ethnic Studies, 37(1), 121-126.

McCracken, Grant. (2008). Transformations: Identity construction in contemporary culture. Bloomington, IN: Indiana University Press.

Myers, C., Clark, M. D., Musyoka, M. M., Anderson, M. L., Gilbert, G. L., Agyen, S., \& Hauser, P. C. (2010). Black deaf individuals' reading skills: Influence of ASL, culture, family characteristics, reading experience, and education. American Annals of the Deaf, 155(4), 449-457.

Napier, J. (2002). The D/deaf-H/hearing debate. Sign Language Studies, 2(2), 141-149.

Nikolopoulos, T. P., O'Donoghue, G., \& Archbold, S. M. (1999). Age at implantation It's implications in paediatric cochlear implantation. Laryngology, 109(4), 595-599.

Niparko, J. K., \& Blankenhorn, R. (2003). Cochlear implants in young children. Mental Retardation and Developmental Disabilities Research Reviews, 9, 267-275.

Norton, B. (2008). Identity, language learning, and critical pedagogies. In J. Cenoz, \& N. $\mathrm{H}$. Hornberger, (Eds.), Encyclopedia of language and education, $2^{\text {nd }}$ Edition, Volume 6: Knowledge about language (pp. 45-57). New York, NY: Springer Science+Business Media, LLC.

Ó Riagáin, P. (2008). Language attitudes and minority languages. In J. Cummins, \& N. $\mathrm{H}$. Hornberger, (Eds), Encyclopedia of language and education, $2^{\text {nd }}$ Edition, Volume 6: Knowledge about language (pp. 329-341). New York, NY: Springer Science+Business Media, LLC. 
Pittam, J. (1999). The Historical and emergent enactment of identity in language. Research on Language and Social Interaction, 31(1\&2), 111-117.

Power, T. (2010). Newfoundland school for the deaf to close. Retrieved July 24, 2011, from http://www.releases.gov.nl.ca/releases/2010/edu/0802n10.htm

Rayman, J. (2009). Why doesn't everyone here speak Sign Language? Questions of language policy, ideology and economics. Current Issues in Language Planning, $10(3), 338-350$.

Reagan, T. (2005). Non-Western educational traditions: Indigenous approaches to educational thought and practice, $3^{\text {rd }}$ ed. Mahwah, NJ: Lawrence Erlbaum.

Reagan, T. (2010). Language policy and planning for sign languages. Washington, DC: Gallaudet University Press.

Reagan, T. (2011). Ideological barriers to American Sign Language: Unpacking linguistic resistance. Sign Language Studies, 11(4), 606-636.

Renick Thomson, N., Kennedy, E. A., \& Kuebli, J. E. (2011). Attachment formation between deaf infants and their primary caregivers: Is being deaf a risk factor for insecure attachment? In D. H. Zand, \& K. J. Pierce, (Eds.), Resilience in deaf children: Adaptation through emerging adulthood (pp. 27-64). New York, NY: Springer Science+Business Media, LLC.

Ross, M. (1993). Personal and social identity of hard of hearing people. Retrieved Aug. 5, 2010, from http://www.ifhoh.org/papers/ross $1 . \mathrm{htm}$

Sampson, E. E. (1991). Social worlds personal lives: An introduction to social psychology. San Diego, CA: Harcourt Brace Jovanovich, Publishers.

Senghas, R. J., \& Monaghan, L. (2002). Signs of theirs times: Deaf communities and the culture of language. Annual Review of Anthroplogy, 31, 69-97.

Simon, B. (2004). Identity in modern society: A social psychological perspective. Malden, MA: Blackwell Publishing Ltd.

Simon, B. (1987). Systematisation and segmentation: The case of England. In D. K. Müller, F. Ringer, \& B. Simon (Eds.), In the rise of the modern educational system: Structural change and social reproduction 1870-1920 (pp. 88-108). New York, NY: Cambridge University Press.

Shilling, C. (2005). The Body in culture, technology and society. London, UK: Sage Publications Ltd.

Shultz, K. (2000). Every implanted child a star (and some other failures): Guilt and shame in the cochlear implant debates. Quarterly Journal of Speech, 86(2), 251275. 
Skutnabb-Kangas, T. (2001). The globalization of (educational) language rights. International Review of Education, 47(3-4), 201-219.

Smith, M. E. \& Campbell, P. (1997). Discourses on deafness: social policy and the communicative habilitation of the deaf. Canadian Journal of Sociology, 22(4), 437456.

Sorkin, D. L. (1996). Developing an identity for people with hearing loss. Retrieved from http://www.ifhoh.org/index.php?option=com_content\&view=article\&id=35:develo ping-an-identity-for-people-with-hearing-loss\&catid=31:general\&Itemid $=1$

Spencer, P. E., \& Marschark, M. (2003). Cochlear implants: Issues and implications. In M. Marschark \& P. E. Spencer (Eds.), Oxford handbook of deaf studies, language and education (pp. 434-448). New York, NY: Oxford University Press.

Statistics Canada. (2008). Participation and Activity Limitation Survey 2006: A profile of education for children with disabilities in Canada. Ottawa, ON: Statistics Canada, Catalogue no. 89-628-X no. 004.

Tanggaard, L. (2009). The Research interview as a dialogical context for the production of social life and personal narratives. Qualitative Inquiry, 15(9), 1498-1515.

Thumann-Prezioso, C. (2005). Deaf parents' perspectives on deaf education. Sign Language Studies, 5(4), 415-440.

Tillmann-Healy, L. M. (2006). Friendship as method. In S. N. Hesse-Biber \& P. Leavy (Eds.), Emergent methods in social research (pp. 273-294). Thousand Islands, CA: Sage Publications Inc.

Urberg, K. A., Degirmencioglu, S. M., \& Tolson, J. M. (1998). Adolescent friendship selection and termination: The role of similarity. Journal of Social and Personal Relationships, 15(5), 703-710.

Wald, R. L., \& Knutson, J. F. (2000). Deaf cultural identity of adolescents with and without cochlear implants. Annals of Otology, Rhinology and Larynogology, 185, s87-s89.

Weiner, M. T., \& Miller, M. (2006). Deaf children and bullying: Directions for future research. American Annals of the Deaf, 151(1), 61-70.

Weisberg, R. (Producer), \& Aronson, J. (Director). (2000). Sound and fury [Motion picture]. United States: New Wave Films

Weiss, R. S. (1998). A Taxonomy of relationships. Journal of Social and Personal Relationships, 15(5), 671-683.

Wheeler, A., Archbold, S., Gregory, S., \& Skipp, A. (2007). Cochlear implants: The young people's perspective. Journal of Deaf Studies and Deaf Education, 12(3), 303-316. 
Woods, D. (1996). Teacher cognition in language teaching: Beliefs, decision-making and classroom practice. Cambridge, UK: Cambridge University Press.

Woodward, K. (2004). Questions of identity. In K. Woodward (Ed.), Questioning identity: Gender, class, ethnicity (pp. 5-42). London, UK: Routledge.

Yoshinaga-Itano, C. (2011). Risk and resiliency of infants/toddlers who are deaf: Assessment and intervention issues. In D. H. Zand, \& K. J. Pierce, (Eds.), Resilience in deaf children: Adaptation through emerging adulthood (pp. 87-111). New York, NY: Springer Science+Business Media, LLC. 


\section{Appendix A: Sample Interview Questions}

Basic information

Name?

Age range? $18-25 ; 26-30 ; 31-35 ; 36-40 ; 41-45 ; 46-50 ; 51-55 ; 56-60 ; 61-65$

Highest level of education?

Hearing status?

Degree of hearing loss?

Personal history

Where were you born?

Where did you grow up?

Are other members of your family deaf?

Can you tell me about your family?

(Looking for information pertaining to the hearing status of other members)

\section{School}

Tell me about your path through elementary and secondary schools.

How did teachers communicate with you?

How did other children communicate with you?

What did you like best about school?

What did you like least about school?

Identity

When did you know you were deaf/different?

(Do you remember any events that lead to you realizing that you were different from others around you?)

Can you tell me about your childhood friends?

And your current friends?

Do you identify more closely with the hearing world or the deaf world?

Why?

Do you see yourself as disabled?

Please explain your answer. 


\section{Appendix B: Sample Interview Transcriptions}

Ben:

[Your favorite part of Deaf school?]

Socializing, meeting all different people. [I did different things every day.] Up until then, it was different. I was in res, I stayed there all week instead of going home to parents every day. In Centennial, that's how it was. At Belleville, you stayed all week, there were no parents to look after the kids, so we just played all the time.

[But there were counselors?]

Yeah, yeah, yeah, there were counselors. Most of the time, we were on our own though.

So, what we'd do, play baseball, go outside and bicycle, chat.

[Weekends?]

Every Friday after school - school closed early - finished at 2:30, normally from Monday

to Thursday, it went to $3,3: 45$. There would be a bus to take you home. So I lived in Ottawa.

[Going back to Deaf school] There were four stops. First stop was St Laurent. That was

me. It was close to the country, St Laurent is East. Then, that was about 5:00, I'd get picked up. After that, to the West End. There were a lot of people there cause it was kind of central. So a lot of people joined there. My stop at St Laurent, there were maybe 6 or 7 people, that's it. But I was lucky because I got to grab my favorite seat on the bus. So then the West End. So many kids, so there was competition to get your seat. It was chaos. Third stop was Kanata, and the fourth stop was Perth or Carleton Place, either one of those two. But most of those people over there, at the fourth stop, most of these kids were hearing students with learning disabilities.

[They went to your Deaf school too?]

Yeah, yeah, (that school) had a smaller section within it for learning disabilities.

[What kind of disabilities?]

I don't know, I was far removed from that. But, yeah, it's the same with all the provincial schools, they have a part for students with learning disabilities. Belleville, Milton, London, no, not London. Belleville and Milton definitely do. Yeah.

[C:? Socializing on weekends?]

No, no, no all week that was enough, the weekend was focused on family stuff.

Sometimes, every so often, there'd be a deaf-event. In Ottawa, a friend would let me know about it, so I'd go, but, yeah, that didn't happen often. Cause my parents had other plans, you know? Family stuff, so you know, [I'd share my time]. I'd have to like plead to go, because we lived in the country, so my dad would have to drive me in, drop me off, then pick me up, drive back. So sometimes I'd sleep over at my friend's, who was close to home. In the summer, there wasn't much socializing. I started for a few years, but after that, I stopped. I started about 6 until about 12, I'd go to deaf summer camp. After that, no, I was too old, it was for young kids, but not for teenagers.

[When did you know you were deaf/different?] [a time when you were different?] I don't know, I was little. There were people around, but I didn't know if I was hearing or deaf. I was little. I didn't know I was different. I just played, mostly, I did not have conversations or stuff. I mostly played, so, yeah, there was not a lot of conversation. Playing, yeah, that was the communication. Until my brother was born. Then it all changed, cause he took off. 
Jessie:

[What was your favorite part of school?] 16:58

Favorite part of school? [laughs] I'm trying to remember... Elementary or high school..?

[In general]. Hmm, I would say friends, some of the friends were - I don't know - they know how to work with me, or somehow have a good relationship, and be helpful. Then that makes me feel better because I don't feel alone. So that's the main part of this whole thing. And... in elementary, you know I didn't really see the efforts that they put towards me, but now when I think about it, it's so much work. And I feel appreciative of that [friends?] yeah friends and EA assistant [what did they do?] and other teachers who are willing to help me out. My friends would talk with me, or show me their notes, on what's going on. Like I would ask them what's going on right now, they would tell me what's happening 18:05, or they would let me copy them... easy. But that's the good thing about friends, some of the friends you just know when they come to you, and how they treat you, you can tell that they're there for you, which is nice. That's the best part of the whole thing, of school.

[What about the part you hated the most?]

Hmm, sometimes the system, or they way how teacher talks, sometimes, like high school, it would be difficult, some teachers can be difficult, and sometimes I would feel alone, like... oh like high school, grade 9, I changed from public school to catholic school, that was hard for me because I leave back - behind my public friends, from public school, and I could tell that from, when I move to a different school, it's less contact... between my friends and me. Then when I tried to make new friends in that school, I was the only deaf girl in that school. So it's a big difference for teachers, big difference for students in the school because they never hear [of] a deaf girl in the school... and that was difficult because in grade 9, they pick on me, kind of, and my teacher doesn't help me out, so I pretty much can't do anything until I know how to make jokes, until I know how to fight back, I guess, let's say. And the problem is I don't know how to make jokes, in language school - I miss that - or how to... make pranks on others. So then, high school was kind of difficult. That's what I hate.

[You said some teachers were 'difficult'; what do you mean by that?] When they don't know how to deal with it, that's the thing. Sometimes... for example grade 9 teacher, if I was being picked on or have some problems, she notice it, but she doesn't help out, she doesn't say to the students to stop, or whatever. And sometimes they're not really thinking that, ok [there/they're] noisy around, it won't be that bad for hearing students, because they can hear, but for me it's harder. It depends on the teacher and their attitude 20:37 [as I say?] and their personality. For some of them, you know that they will [work it], and they will be great support.

[In grade 10 and later... did it start to fall into place?]

$\mathrm{Hmm}$, grade 9, grade $10 \ldots$ it was so-so. But I went to a trip to Poland in the summer after grade 10, and I start to learn more about myself. My cousin who were able to speak English, they take me around, they tell me all these things about high school, they tell me all the jokes or whatever. And they say, who cares what you do, like it's who you are, if you are like that, that's who you are, who cares? And I start to realize about it, and I change myself, like I change my hair colour, I change my style of clothes, then when I come back in grade 11, I was a little bit more better and sometimes people who knew me from the classes - I get good marks, I am very nice, I talk a lot, or whatever - they start to 
trust me and they start to allow me - accept me, even. Then in grade 11 and grade 12, I started to join sports, I start to go with friends, meet friends - like I said, I'm still floating around in high school; I'm not in one group, I go to different groups. Even in my class, I talk to this one person, and I say, I meet you at lunch, and then I go to this person, then I go to another friend, and another friend... joining sports and different shows, like Christmas show and talent show. By grade 12, I start to have better friends, and [...22:27] I start to go out more, like I start to go to friend's house, social gathering, and back then I didn't. Not in whole high school, just grade 12. So umm, yeah, it was good. Yeah, I don't stay in one group, like punk or whatever... sometimes some friends have problem with that, but still. [... 23:10]. In grade 12, I started to learn more about jokes, I started to watch more TV shows and I started to catch some things. And when I watch TV shows, there's closed captioning, which helps me the most... because with more understanding of the language, with more understanding of what that situation is talking about, then I can be able to relate it to my situation nowadays, then I can say the jokes too... and then my brother, tells me, if you're in that situation, you have to have the right time of saying it; like if you say that joke in a different time, that's stupid.

Amy:

[any language delays?]

Surprisingly no. But, funny story, I kinda had a language all my own when I was a kid and my parents did not understand why, until I was a little older and they realized well, yeah, I am hard of hearing. Like I had new words. Like... instead of... spaghetti, I used to say spaditi and noodles for noonles. Like I had a dozen of these. And my parents just thought it was funny until they said it to the doctor and he's like, yeah, she's not hearing the proper pronunciation.

[speech therapist?]

I didn't actually. I think it was just in time it... it went away, and because my right ear has always been so fine, it's always been perfect, like that ear somehow was never affected... even though I had the ear infections in that one too. So that one sort of compensated even at the balance?, so I never had speech therapy...

[did not affect communication with parents?]

No... (laughter, unclear)... lots of repetitions around the house, but that's pretty much it. [school?]

Well, elementary school, I don't remember any real challenges. But I mean high school I think was different, because I went through - well I went to the same high school from grade seven to twelve - and grade eight was when I got my hearing aid, the first one. So grade eight, nine, even ten, I think ten was when I stopped hearing?. Grade eight and nine were good, it's funny because I guess I didn't identify as, like, having a real hearing problem until I had that hearing aid. And that's when the school was like, oh, ok, well I guess you should have some sort of accommodations. So then it was all, like, well, for French test, you know, you do like a test d'écoute, a listening test, well, yeah, I got my own nice pair of earphones to put on. Big honking earmuffs. Yeah, it was cool, it was very stylish in a class of thirty people. So I got to sit there and listen to the test as many times as I wanted to... that kind of thing... What else?... Music... it was really funny, because I always had good marks in music, and I had grown up... I love music. I play(played?), I play (ed?) [around 11:42] guitar, I played saxophone and when it got to 
my music teacher that I was hard of hearing, and it was oh yeah she needs accommodations, my teacher like, she was like what are you talking about, she's not hard of hearing (laughter). Yep. I think it was more like a shock element, actually, among most of the staff when that came around cause it's a small school, and I was an ok student I guess. So, no, I did not really struggle I would say, I think, it was more just, there would be things that I would miss, like that, the last minute, like everyone is packing up to leave class and you know the shuffling papers and the teacher "Oh, by the way... something mumble, mumble ... due... " What's due next week? I don't know, because I miss that kind of stuff. And that's when I think it started really becoming evident. There was like, there was actually an assignment or two that I missed a deadline on, and I was a straight A student and it was, like, what happened? What I thought it was due the week after. So, there were times like that I think were ... ?[12:47] for no reason, that type of thing.

Steph:

And now... now that I'm learning about the Deaf community and sign language here, this is the first time I've actually.... Known what the Deaf community... is and what it's about, and stuff... I'm like, oh - cool! So it's still a very new idea for me, but I do like the idea. However, I think in order to be part of a community you have to participate in it and... frequently. Like... well... I'm heavily involved in the engineering community still - I do sort of see some... I, I often compare similarities between the engineering community and the Deaf community, especially since we give each other nicknames type thing, like sign names... They have [call signs?]... but they're usually terrible nicknames too... as in the more inappropriate, the better - right so, you see people that are heavily involved in the community, like, yeah, they're engineering community people, whereas there's some people that'll show up to like one or two things, [misunderstood]. So, right now, as far as the Deaf community goes, I sort of feel like... I don't know, like, yes, I've gone out to a few things, but that was like, two years ago, almost now, and... I don't... know these people very well or [whatnot?] - I don't know, I think I'd like to... But I think I'd have to participate a lot more in the community, and... improving my sign language would help... and so like... if I get my sign language up to a level where I'm happy with it, and use it a lot more often than I do now... obviously I'm not using it a whole lot(!)... yeah so if I tend to use sign language more often and more frequently and the quality improves and I make it out and socialize with the Deaf community, then yeah, I'll probably consider myself to have joined it, but right now... I'm still an outsider. I don't know when their events start... Like it would be like someone in the engineering community - like, for the engineering community, I know when things are gonna happen, who's involved, who to ask about things: I'm part of it. Whereas with the Deaf community right now, I'm sort of like... I can ask someone to ask someone to ask someone... [...] So I'm not really part of the community just yet, but we'll see. [Do you want to be - do you feel like that's a place for you?] (Sighs) See that's a tough one, because.... I love my hearing friends, I'm never gonna leave them, obviously... but at the same time, there are things that make me feel more accepted with deaf people, because we can relate to different things - well I mean, obviously I can't relate to being deaf-deaf... I've always done well in school and whatnot... but - I remember when I first started sign language, Jon's like - when he first saw me, he's like, oh god, another hard-of-hearing person in the class, probably just 
gonna drop out... and, he said that he was really impressed that I like picked it up and actually answered things correctly....

[Ok, so you don't see yourself as 'true-work' deaf, but not hearing either...]

Yeah-

[You're kind of in the middle...?]

... Well I mean, I can't say for sure how I view the Deaf community - I'm not really a part of it right now - I'd have to experience it first, before I could have a solid opinion about it... I've lived my whole life in the hearing community, so I know how hearing people think, and I think that-like, along the same lines, right? I mean, you go to school, you get a job, you get married, you have kids... I never thought of different things, like it's the path I know; it's the path I grew up being taught... but I consider myself openminded, but I have to... I have to expe-I need to get more knowledge about it, I need to know more about something before I can make an informed decision.

[How do you think you identify more with deaf people than with hearing people?] $~ 37: 00$ Ummm, not necessarily more - differently. Like, in lectures, when I'm focusing, paying attention to the prof, it takes a lot of energy, like, I'm trying, I'm trying... and Ryan's like, well, yeah, it's because you can't hear it - it's hard!, and I'm like... you know, that's probably it! And... little things like that... and I can relate to them on some levels, and it - it's nice cause I always felt different for I don't know what reason, like I guess... it's sort of like I accepted being 'a daydreamer', I accepted being 'someone who falls asleep in lectures', when really it's... like until I met other deaf people - it hadn't even crossed my mind. [...] So that's the reason I'm focusing more on the note-takers now, because that way, I can still focus on lectures, but if I get really tired, I can look away for a second and... put my mind on something else for 2 seconds then focus back. Sort of give myself a rest period without completely falling asleep in front of the prof and in front of the class. Oh! Once I had the FM system on in class and I fell asleep and he's like, 'Steph' [gesture: speaking into microphone]

Joey:

[your experience after the operation...] 16:16

- I have a ringing in my ears, and I've had it since I was young, it's sort of like a constant squeal, like the feedback from a microphone or from a hearing aid... and when I first had the implant turned it on, I thought it was that squeal, it was just a high pitched tone, and then as people started talking I noticed a fluctuation of the 'eeeee'-sort of sound, and eventually started matching it to - well not eventually, within a couple minutes I was matching it to the movement of people's lips... and =, just sort of recognizing that tone, and by the time I was driving home after the switch-on, =, I was noticing sounds and recognizing them [New sounds?] There weren't necessarily any sounds like that but some sounds, I think, I hadn't heard enough of them before, so they sounded very different to me.... =, I remember being very irritated on the drive home, $=$, by the click of the turn signal on the car, just cause it's so much louder, to my senses, than like speech, even. =, and my mom and 1 stopped at the grocery store on the way home and, the rustling of plastic bags was just like... like nails on a chalk board, almost. $=$, so there was a bunch of sounds like that, I think the higher pitch sounds especially... =, just stood out to me. [ever a time you felt regretful or scared] 
- I think in the first week, it was a little... I think I was a little depressed, just... I was debating whether or not it was worth it, I still hadn't grasped the, full breadth of the sound, I wasn't recognizing everything, $=\ldots$ so I think, just,... I remember being on the bus on the way to one of the appointments, follow-up.. tune-up, and just like, just not feeling very good about it, feeling like the cord of the side of my head just stood out, $=\ldots$ but that went away fairly quickly.

-- Just adding/clarifying. The bit of depression I was feeling on that bus ride went away by the end of that follow-up appointment. I had the same kind of single-word and repeating sentences in varying amounts of noise test that I mentioned earlier. At that appointment a week after switch-on, my scores with the implant +one hearing aid were better than with the two hearing aids. I cheered up pretty quickly after that. [do you know anything about the Deaf community?]

,I have a passing knowledge... I don't know anybody from the Deaf community, really. So what I know is basically second hand knowledge, from auditory-verbal therapists, who do have a somewhat biased perspective... and from some fellow students here, which I've met in the past semester, who are taking sign language courses.

[Want to get in [to the deaf community]?]

- It's never really come up... I've encountered (sort of) 2 individuals who were part of the deaf community, but it was a negative experience, I know in retrospect that they were probably extremists, in terms of how they interacted,

\section{Shayna:}

C: Did you have contact with other HoH/deaf children while growing up?

A: Yes, I went to a special class and some of the other students there were deaf and hardof-hearing, but at the same time, there were also mentally retarded students in that class. Also blind students. Just kids with a lot of different needs, so it wasn't a community well, a disabled community in general - no Deaf identity. We didn't use Sign language. There was another boy who used an FM system [describes gesturally what it looks like], yeah he wore that. His therapist didn't let him lip-read; she forced him to depend on speech during practice. So, yeah, I had experience with other deaf kids but he didn't identify as Deaf. We were mainstreamed.

$\mathrm{C}$ : Did you socialize with those kids outside school?

A: His parents didn't encourage us to play together, they wanted to keep us separated. So I'd ask him if he could come and play and he'd say no, that his parents had told him we couldn't play together. I didn't understand, he said his parents said communication would be too hard. So we were separated, it was hard. (32:09) [shrugs]. At the same time, I wanted other friends. It didn't matter who, just other people. So I tried to separate myself from the disabled class, I rejected the idea that I was disabled, I wanted regular friends so I tried to make some and stay out of the disabled group... I didn't really fit in either group, I was always in the middle.

C: Did you take your hearing aid off?

A: Yeah, suppose I was wearing it and got a headache, I'd take it off. My teacher would notice and tell me to put it back on. But then I'd put my head down on the desk and sleep (33:07), so instead of learning, I'd tune out and sleep. You know, it was hard... it didn't help me. It was hard.

C: How do you feel about the hearing world? How do you feel about the Deaf world? 
A: Well, I... hearing [world?], I always tried to fit in, but it didn't quite work, it didn't feel right. I had to work more... so often, hearing people don't understand me - like, with you, it's nice, I'm hard of hearing and you understand and accept that, you're ready to help, it's fine. But you and other people like that, it's special C: ? [cut in] (something about straddling both worlds) A: Yeah, right, same for me, I kind of sit on the fence, but for me, I have one foot in each world. But I'm nervous. My family - I identify as Deaf, I feel that more - but them, they don't want me to announce that to the world. My mom tells me I'm hard of hearing. When I was growing up, my mom told me I was hard of hearing; she tried to decide that for me. So when I was little, it was awkward but I went along with it, tried to oblige [?]

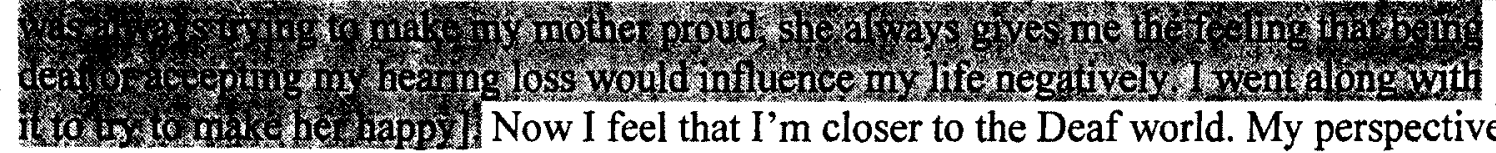
is really different from [the hearing world's/my mother's]. I disagree with the hearing world: often hearing people feel that cochlear implants are the best answer, that every [deaf] child should be implanted. I disagree. My - well, now my boyfriend, his name is [Laurent], he's deaf, he wears hearing aids in both ears - his family is the same as mine. They both think 'hearing'. Last week, I went to visit him - it was the first time I was meeting his family - and he told me not to sign. He and I always sign! But he told me, 'no, no, don't sign; speak'. Why? Well, his family had asked him if I was deaf or hard of hearing and he told them hard of hearing - they were relieved. So he asked me not to tell them that I would become deaf later [in life]. I said, 'but it's the truth! I don't want to lie to your family, they should know the truth'. - 'it's complicated'. I don't want to be fake, to pretend. I tried to explain that but he didn't understand. We fought about it. So it's hard. Deaf identity, sometimes people want it (end of video 2) - 


\section{Appendix C: Elaborated Glossing}

The below diagram is an example of elaborated glossing. As one can observe, the representation of a signed sentence is made up of three levels. The line above the glosses represents the grammatical features of the sentence, which mostly consist of facial expressions and/or non-manual elements (for example, frowns, forward body leaning, lippursing). The middle line represents those signs produced with the dominant hand, or with both hands when a sign requires it. The bottom line, formatted in italics, represents the actions of the non-dominant hand. This last line is somewhat optional as its part is normally supportive in nature. In other words, the non-dominant hand tends to be used to index spatial locations, above other functions, which is not mandatory during conversation.

Glosses are represented by words spelled with uppercase letters. Those words that appear in lowercase italics represent non-manual information, such as spatial locations, or they may reference additional morphological information. The + symbol is used to represent repetition of a sign, while the \# symbol refers to a loan sign.

neg

\#NO+, GROUP-lfCHAT-lf, GROUP-cntr DANCE-cntr ENJOY-cntr, GROUP-rt "WELL" AWKWARD-rt, SHY-rt, DIFFERENT+++-arc

INDEX-rt

This sentence translates approximately into, "No, there were all sorts [of people]. Some chatting, some dancing and having fun, and others were awkward and shy". For a more detailed description of these elements, see Baker-Shenk, C., \& Cokely, D. (1980, pp.129). American Sign Language: A Teacher's Resource Text on Grammar and Culture. Washington, DC: Gallaudet University Press. 